\title{
PERFECT COMPLEXES ON ALGEBRAIC STACKS
}

\author{
JACK HALL AND DAVID RYDH
}

\begin{abstract}
We develop a theory of unbounded derived categories of quasicoherent sheaves on algebraic stacks. In particular, we show that these categories are compactly generated by perfect complexes for stacks that either have finite stabilizers or are local quotient stacks. We also extend Toën and Antieau-Gepner's results on derived Azumaya algebras and compact generation of sheaves on linear categories from derived schemes to derived DeligneMumford stacks. These are all consequences of our main theorem: compact generation of a presheaf of triangulated categories on an algebraic stack is local for the quasi-finite flat topology.
\end{abstract}

\section{INTRODUCTION}

Our first main result is the following.

Theorem A. Let $X$ be a quasi-compact algebraic stack with quasi-finite and separated diagonal. Then the unbounded derived category $\mathrm{D}_{\mathrm{qc}}(X)$, of $\mathcal{O}_{X}$-modules with quasi-coherent cohomology, is compactly generated by a single perfect complex. Moreover, for every quasi-compact open subset $U \subseteq X$, there exists a compact perfect complex with support exactly $X \backslash U$.

This generalizes the results of A. Bondal and M. Van den Bergh [BB03, Thm. 3.1.1] for schemes and B. Toën [Toë12, Cor. 5.2] for Deligne-Mumford stacks admitting coarse moduli spaces (i.e., $X$ has finite inertia). While we can show that every compact object of $\mathrm{D}_{\mathrm{qc}}(X)$ is a perfect complex (Lemma 4.4), a subtlety in Theorem $\mathrm{A}$ is that the converse does not always hold in positive characteristic. Indeed, if $X$ is not tame, then the structure sheaf is perfect but not compact.

Theorem A, as well as the theory developed to establish it, have been used to classify the thick tensor ideals in $\mathrm{D}_{\mathrm{qc}}(X)^{c}$ Hal16] (generalizing work of [Kri09, DM12]), to resolve the telescope conjecture for algebraic stacks [HR17] (extending Ant14]), and for results on dg-enhancements [CS16, BLS16.

Extending Theorem A to certain stacks with infinite stabilizer groups is our second main result. We briefly recall a notion from [Ryd15, §2]: an algebraic stack $X$ is of $s$-global type if étale-locally it is the quotient of a quasi-affine scheme by $\mathrm{GL}_{N}$ for some $N$.

Theorem B. Let $X$ be an algebraic stack of s-global type. If $X$ is of equicharacteristic zero (i.e., it is a $\mathbb{Q}$-stack), then the unbounded derived category $\mathrm{D}_{\mathrm{qc}}(X)$ is compactly generated by a countable set of perfect complexes. Moreover, for every quasi-compact open subset $U \subseteq X$, there exists a compact perfect complex with support exactly $X \backslash U$.

Date: May 24, 2017.

2010 Mathematics Subject Classification. Primary 14F05; secondary 13D09, 14A20, $18 \mathrm{E} 30$.

Key words and phrases. Derived categories, algebraic stacks, compact generation, perfect complexes.

This collaboration was supported by the Göran Gustafsson foundation. The first author was supported by the Australian Research Council DE150101799. The second author is supported by the Swedish Research Council 2011-5599. 
Stacks of s-global type are frequently encountered in practice. Sumihiro's Theorem [Sum74] and its recent generalization by Brion [Bri15] show that many quotient stacks are of s-global type (Proposition 9.1). Thus, we have the following corollary (see Corollary 9.2 for an amplification).

Corollary. Let $X$ be a variety over a field $k$ of characteristic zero. Let $G$ be an affine algebraic $k$-group acting on $X$. If $X$ is either (a) normal or (b) seminormal and quasi-projective, then $\mathrm{D}\left(\mathrm{QCoh}_{G}(X)\right)=\mathrm{D}_{\mathrm{qc}}([X / G])$ is compactly generated. Moreover, for every $G$-invariant open subset $U \subseteq X$, there exists a perfect $G$ equivariant complex with support exactly $X \backslash U$.

A key advantage of Theorem B over previous results (e.g., BZFN10, Cor. 3.22] and [Lie04, Prop. 2.2.4.13]) is its applicability to a much wider class of stacks. The main result of [AHR15] implies that algebraic stacks of finite type over a field with affine diagonal and linearly reductive stabilizers at closed points (e.g., stacks with a good moduli space) are of s-global type [AHR15, Thm. 2.25]. In general, it is not possible to generate $\mathrm{D}_{\mathrm{qc}}(X)$ by a single perfect complex (e.g., $X=B \mathbb{G}_{m}$ ).

Theorems $\mathrm{A}$ and $\mathrm{B}$ are both consequences of a general result that we now describe. Let $\beta$ be a cardinal. Let $X$ be an algebraic stack. We say that $X$ satisfies the $\beta$-Thomason condition if:

(1) $\mathrm{D}_{\mathrm{qc}}(X)$ is compactly generated by a set of cardinality $\leq \beta$; and

(2) for every quasi-compact open subset $U \subseteq X$, there is a compact perfect complex supported on the complement.

We say that $X$ satisfies the Thomason condition if it satisfies the $\beta$-Thomason condition for some $\beta$. Our main results are that a very large class of stacks satisfy the Thomason condition. In order to prove these results, however, we found it necessary to consider the following refinement of the Thomason condition.

We say that $X$ is $\beta$-crisp if the $\beta$-Thomason condition is satisfied for every étale localization of $X$ (Definition 8.1). If $X$ is $\beta$-crisp and $\beta$ is finite, then $X$ is compactly generated by a single perfect complex. Hence Theorems $\mathrm{A}$ and $\mathrm{B}$ are implied by

Theorem C. Let $p: X^{\prime} \rightarrow X$ be a morphism of quasi-compact and quasi-separated algebraic stacks that is representable, separated, quasi-finite, locally of finite presentation, and faithfully flat. If $X^{\prime}$ is $\beta$-crisp, then $X$ is $\beta$-crisp.

Theorem $\mathrm{C}$ is proved using the technique of quasi-finite flat dévissage for algebraic stacks - due to the second author Ryd11 — together with some descent results for compact generation. In sections 56 these descent results are stated in great generality - for presheaves of triangulated categories - without requiring monoidal or linear structures. This allows us to establish compact generation in other contexts (see below, Theorem 6.9, and \$9). Along the way, we will review and develop foundational material for unbounded derived categories on algebraic stacks.

We also wish to point out that for schemes the fppf and quasi-finite flat topologies coincide, but for algebraic stacks they differ. Moreover, compact generation is not fppf local for algebraic stacks. Indeed, $\mathrm{D}_{\mathrm{qc}}\left(B_{k} \mathbb{G}_{a}\right)$ when $k$ is of characteristic $p>0$ has no compact objects besides 0 ; thus, it is not compactly generated - even though it is so fppf-locally HNR14, Prop. 3.1]. In particular Theorem C (as well as its generalizations to other contexts in this article), which is about quasi-finite local compact generation, can be viewed as the correct generalization of fppf local compact generation results to algebraic stacks.

Azumaya algebras and the cohomological Brauer group. Our work is strongly influenced by Toën's excellent paper Toë12 on derived Azumaya algebras and generators of twisted derived categories. In Toë12, Toën shows that compact generation of certain linear categories on derived schemes is an fppf-local question. 
The salient example is the derived category of twisted sheaves $\mathrm{D}\left(\mathrm{Q} \operatorname{Coh}^{\alpha}(X)\right)$, where the twisting is given by a Brauer class $\alpha$ of $H^{2}\left(X, \mathbb{G}_{m}\right)$. A compact generator of $\mathrm{D}\left(\mathrm{Q} \operatorname{Coh}^{\alpha}(X)\right)$ gives rise to a derived Azumaya algebra - the endomorphism algebra of the generator [Toë12, Prop. 4.6]. More classically, a twisted vector bundle that is generating gives rise to an Azumaya algebra $\mathcal{A}$.

The Brauer group $\operatorname{Br}(X)$ classifies Azumaya algebras $\mathcal{A}$ up to Morita equivalence, that is, up to equivalence of the category of modules $\operatorname{Mod}(\mathcal{A})$. Moreover, $\operatorname{Mod}(\mathcal{A}) \simeq \mathrm{Q} \operatorname{Coh}^{\alpha}(X)$ for a unique element $\alpha$ in the cohomological Brauer group $\operatorname{Br}^{\prime}(X):=H^{2}\left(X, \mathbb{G}_{m}\right)_{\text {tors }}$. Existence of twisted vector bundles thus answers the question whether $\operatorname{Br}(X) \rightarrow \operatorname{Br}^{\prime}(X)$ is surjective.

Constructing twisted vector bundles, or equivalently Azumaya algebras, is difficult. Indeed, the question is not local as vector bundles rarely extend over open immersions. When $X$ is affine, or the separated union of two affines, Gabber proved in his thesis that $\operatorname{Br}(X)=\operatorname{Br}^{\prime}(X)$ Gab81, or equivalently, that $\mathrm{D}\left(\mathrm{Q} \operatorname{Coh}^{\alpha}(X)\right)$ is compactly generated by a twisted vector bundle [Lie04, Thm. 2.2.3.3]. The state of the art is also due to Gabber: twisted vector bundles exist if $X$ is quasi-projective Jon03.

Compact objects of the derived category are typically easier to construct as we may extend them over open immersions using Thomason's localization theorem (Corollary 3.13). With this technique, M. Lieblich proved that $\mathrm{D}\left(\mathrm{Q} \operatorname{Coh}^{\alpha}(X)\right.$ ) is compactly generated when $X$ is any quasi-compact and quasi-separated scheme Lie04 Cor. 2.2.4.14]. Lieblich has also studied twisted vector bundles in great detail and obtained a number of arithmetic applications.

In $\$ 9$. we prove that compact generation is quasi-finite flat local for twisted derived categories. In particular, we prove that on a quasi-compact algebraic stack with quasi-finite and separated diagonal every twisted derived category has a compact generator (Example 9.3). We thus establish a derived analogue of $\operatorname{Br}(X)=\operatorname{Br}^{\prime}(X)$ for such stacks, extending the results of Toën Toë12] and AntieauGepner AG14.

Sheaves of linear categories on derived Deligne-Mumford stacks. Although we work with non-derived schemes and stacks, our methods are strong enough to deduce similar results for derived (and spectral) Deligne-Mumford stacks (Example 9.4). Indeed, if $X$ is a derived Deligne-Mumford stack, then the small étale topos of $X$ is equivalent to the small étale topos of the non-derived 0 -truncation $\pi_{0} X$. Thus, (local) compact generation of a presheaf of triangulated categories on $X$ can be studied on $\pi_{0} X$.

Sometimes results for stacks can be deduced from schemes using a similar approach: if $\pi: X \rightarrow X_{\mathrm{cms}}$ is a coarse moduli space, then a presheaf $\mathcal{T}$ of triangulated categories on $X$ induces a presheaf $\pi_{*} \mathcal{T}$ of triangulated categories on $X_{\mathrm{cms}}$. If $\pi_{*} \mathcal{T}$ is locally compactly generated, then it is enough to show that compact generation is local on $X_{\text {cms }}$ to deduce compact generation of $\mathcal{T}(X)$. This is how Toën extends his result to Deligne-Mumford stacks admitting a coarse moduli scheme [Tö̈12, Cor. 5.2].

Perfect and compact objects. As we already have mentioned, some care has to be taken since perfect objects are not necessarily compact. The perfect objects are the locally compact objects or, equivalently, the dualizable objects. If $X$ is a quasi-compact and quasi-separated algebraic stack, then the following conditions are equivalent (Remark 4.6):

- every perfect object of $\mathrm{D}_{\mathrm{qc}}(X)$ is compact;

- the structure sheaf $\mathcal{O}_{X}$ is compact; 
- there exists an integer $d_{0}$ such that for all quasi-coherent sheaves $M$ on $X$, the cohomology groups $H^{d}(X, M)$ vanish for all $d>d_{0}$; and

- the derived global section functor $\mathrm{R} \Gamma: \mathrm{D}_{\mathrm{qc}}(X) \rightarrow \mathrm{D}(\mathrm{Ab})$ commutes with small coproducts.

We say that a stack is concentrated when it satisfies the conditions above.

In [HR15, Thm. B] we give a complete list of the group schemes $G / k$ such that $B G$ is concentrated: every linear group and certain non-affine groups in characteristic zero but only the linearly reductive groups in positive characteristic. Note that [HR15, Thm. A] also gives many examples of classifying stacks that are not concentrated, yet compactly generated.

Drinfeld and Gaitsgory have proved that noetherian algebraic stacks with affine stabilizer groups in characteristic zero are concentrated [DG13, Thm. 1.4.2]. This is generalized in [HR15, Thm. C] to positive characteristic. In particular, a stack with finite stabilizers is concentrated if and only if it is tame.

Perfect stacks. Ben-Zvi, Francis and Nadler introduced the notion of a perfect (derived) stack in BZFN10. In our context, an algebraic stack $X$ is perfect if and only if it has affine diagonal, it is concentrated and its derived category $\mathrm{D}_{\mathrm{qc}}(X)$ is compactly generated [BZFN10, Prop. 3.9]. A direct consequence of our main theorems and [HR15, Thm. C] is that the following classes of algebraic stacks are perfect:

(1) quasi-compact tame Deligne-Mumford stacks with affine diagonal; and

(2) $\mathbb{Q}$-stacks of s-global type with affine diagonal.

The affine diagonal assumption is needed only because it is required in the definition of a perfect stack. It is useful though: if $X$ is perfect, then $\mathrm{D}(\mathrm{Q} \operatorname{Coh}(X))=\mathrm{D}_{\mathrm{qc}}(X)$ by HNR14.

In the terminology of Lurie [DAGXI, Def. 8.14], an algebraic stack is perfect if it has quasi-affine diagonal, is concentrated and $\mathrm{D}_{\mathrm{qc}}(X)$ is compactly generated. Thus in Lurie's terminology, we have shown that

(1) quasi-compact tame Deligne-Mumford stacks with quasi-compact and separated diagonals; and

(2) $\mathbb{Q}$-stacks of s-global type are perfect.

Coherence. Compact generation is extremely useful and we will illustrate this with a simple application - also the origin of this paper. Let $A$ be a commutative ring and $\operatorname{Mod}(A)$ the category of $A$-modules. A functor $F: \operatorname{Mod}(A) \rightarrow \mathrm{Ab}$ is coherent if there exists a homomorphism of $A$-modules $M_{1} \rightarrow M_{2}$ together with isomorphisms

$$
F(N) \cong \operatorname{coker}\left(\operatorname{Hom}_{A}\left(M_{2}, N\right) \rightarrow \operatorname{Hom}_{A}\left(M_{1}, N\right)\right)
$$

natural in $N$. This definition is due to Auslander Aus66 who initiated the study of coherent functors. Hartshorne studied in detail Har98. coherent functors when $A$ is noetherian and $M_{1}$ and $M_{2}$ are finitely generated and obtained some very nice applications to classical algebraic geometry. For background material on coherent functors, we refer the reader to Hartshorne's article. Recently, the first author has used coherent functors to prove Cohomology and Base Change for algebraic stacks Hal14] and to give a new criterion for algebraicity of a stack [Hal17.

Using the compact generation results of this article, we can give a straightforward proof of the following Theorem (combine Theorem A with Corollary 4.16).

Theorem D. Let $A$ be a noetherian ring and let $\pi: X \rightarrow \operatorname{Spec} A$ be a proper morphism of algebraic stacks with finite diagonal. If $\mathcal{F} \in \mathrm{D}_{\mathrm{qc}}(X)$ and $\mathcal{G} \in \mathrm{D}_{\text {Coh }}^{b}(X)$, 
then the functor

$$
\operatorname{Hom}_{\mathcal{O}_{X}}\left(\mathcal{F}, \mathcal{G} \otimes_{\mathcal{O}_{X}}^{\mathrm{L}} \mathrm{L} \pi_{\mathrm{qc}}^{*}(-)\right): \operatorname{Mod}(A) \rightarrow \operatorname{Mod}(A)
$$

is coherent.

Theorem D generalizes a result of the first author for algebraic spaces Hal14, Thm. E], which was proved using a completely different argument. The first author has also proved a non-noetherian and infinite stabilizer variant of Theorem $\mathrm{D}$ at the expense of assuming that $\mathcal{G}$ has flat cohomology sheaves over $S$ [Hal14, Thm. C].

Related results. The first proof that $\mathrm{D}_{\mathrm{qc}}(X)$ is compactly generated when $X$ is a quasi-compact separated scheme appears to be due to Neeman [Nee96, Prop. 2.5] although he attributes the ideas to Thomason [TT90. Bondal-Van den Bergh BB03, Thm. 3.1.1] adapted the proof to deal with quasi-separated schemes and noted that there is a single compact generator. Lipman and Neeman further refined the result by giving an effective bound on the existence of maps from the compact generator [LN07, Thm. 4.2]. As noted by Ben-Zvi, Francis and Nadler, the proof of Bondal and Van den Bergh readily extends to derived schemes [BZFN10, Prop. 3.19].

In DAGXI, Thm. 6.1] and DAGXII, Thm. 1.5.10] Lurie proves that compact generation is étale local on $\mathbb{E}_{\infty}$-algebras and on spectral algebraic spaces for quasicoherent stacks (sheaves of linear $\infty$-categories). Lurie uses scallop decompositions, which are a special type of étale neighborhoods (or Nisnevich squares). Unfortunately, scallop decompositions do not exist for algebraic stacks. This is what necessitates our stronger inductive assumption- $\beta$-crispness - for our local-global principle, Theorem C. Indeed, to apply Thomason's localization theorem it is necessary to establish the existence of compact objects with prescribed support. On affine schemes (which appear in the scallop decompositions) this is done using Koszul complexes, cf. Bökstedt and Neeman [BN93, Prop. 6.1]. This is the basis for our induction and also used in all previous proofs, e.g., Toën [Toë12, Lem. 4.10] and AG14, Prop. 6.9].

Drinfeld and Gaitsgory [DG13, Thm. 8.1.1] prove that on an algebraic stack of finite type over a field of characteristic zero with affine stabilizers, the derived category of $D$-modules is compactly generated. They remark that compact generation of $\mathrm{D}_{\mathrm{qc}}(X)$ is much subtler and open in general [DG13, 0.3.3].

Antieau Ant14 has considered local-global results for the telescope conjecture. Some of these are generalized in HR17.

Krishna Kri09 has considered the K-theory and G-theory for tame DeligneMumford stacks with the resolution property admitting projective coarse moduli schemes (i.e., projective stacks).

Future extensions. In [LN07, Thm. 4.1], Lipman and Neeman prove that pseudocoherent complexes can be approximated arbitrarily well by perfect complexes on a quasi-compact and quasi-separated scheme. Local approximability by perfect complexes is essentially the definition of pseudo-coherence so this is a local-global result in the style of Theorem C. This result has been extended to algebraic spaces in $[$ Stacks, $08 \mathrm{HH}$ ] and we expect that it can be extended to stacks with quasi-finite diagonal using the methods of this paper amplified with $t$-structures. Similarly, we expect that there is an effective bound on the compact generator in Theorem $\mathrm{A}$ as in [LN07, Thm. 4.2].

Contents of this paper. In $\S \S 1-2$ we recall and develop some generalities on unbounded derived categories of quasi-coherent sheaves on stacks and concentrated morphisms - working in the unbounded derived category is absolutely essential for this range of mathematics. Unfortunately some important foundational notions, such as concentrated morphisms, had not been considered in the literature before. 
In $\S 3$, we recall the concept of compact objects and Thomason's localization theorem for triangulated categories.

In $\S 4$, we address fundamental results on perfect and compact objects in the derived categories of quasi-coherent sheaves on algebraic stacks. Using this we establish a general projection formula for stacks, tor-independent base change, and finite flat duality. We also prove Theorem $\mathrm{D}$ assuming Theorem A.

In $§ \S 5-6$ we introduce presheaves of triangulated categories and Mayer-Vietoris triangles. We also prove our main result on descent of compact generation (Theorem 6.9.).

In $\S 7$ we introduce the $\beta$-resolution property, which gives a convenient method to keep track of the number of vector bundles needed for generating the derived category of a stack with the resolution property.

In $\S 8$, we introduce compact generation with supports and $\beta$-crispness and relate these to Koszul complexes.

In $\S 9$, we prove the main theorems.

Acknowledgments. It is our pleasure to acknowledge useful discussions with B. Bhatt and A. Neeman. We would also like to thank D. Bergh, M. Hoyois, M. Lieblich and O. Schnürer for a number of useful comments. We also wish to thank the referee for their careful reading and several suggested improvements to the article.

Notations and assumptions. For an abelian category $\mathcal{A}$, denote by $\mathrm{D}(\mathcal{A})$ its unbounded derived category. For a complex $M \in \mathrm{D}(\mathcal{A})$, denote its $i$ th cohomology group by $\mathcal{H}^{i}(M)$. For a sheaf of rings $A$ on a topos $E$, denote by $\operatorname{Mod}(A)$ (resp. QCoh $(A))$ the category of $A$-modules (resp. the category of quasi-coherent $A$-modules). If the sheaf of rings $A$ on the topos $E$ is implicit, it will be convenient to denote $\operatorname{Mod}(A)$ as $\operatorname{Mod}(E)$ and $\mathrm{D}(\operatorname{Mod}(A))$ as $\mathrm{D}(E)$.

For algebraic stacks, we adopt the conventions of the Stacks Project Stacks]. This means that algebraic stacks are stacks over the big fppf site of some scheme, admitting a smooth, representable, surjective morphism from a scheme (note that there are no separation hypotheses here). A morphism of algebraic stacks is quasiseparated if its diagonal and double diagonal are represented by quasi-compact morphisms of algebraic spaces.

For a scheme $X$ denote its underlying topological space by $|X|$. For a scheme $X$ and a point $x \in|X|$, denote by $\kappa(x)$ the residue field at $x$.

Let $f: X \rightarrow Y$ be a 1-morphism of algebraic stacks. Then for any other 1morphism of algebraic stacks $g: Z \rightarrow Y$, we denote by $f_{Z}: X_{Z} \rightarrow Z$ the pullback of $f$ by $g$.

\section{QUASI-COHERENT SHEAVES ON ALGEBRAIC STACKS}

In this section we review derived categories of quasi-coherent sheaves on algebraic stacks. For generalities on unbounded derived categories on ringed topoi we refer the reader to [KS06, §18.6]. In [KS06, §18.6], a morphism of ringed topoi is assumed to have a left exact inverse image - we will not make this assumption, but instead indicate explicitly when it does and does not hold.

Let $X$ be an algebraic stack. Let $\operatorname{Mod}(X)(\operatorname{resp} . \mathrm{Q} \operatorname{Coh}(X))$ denote the abelian category of $\mathcal{O}_{X}$-modules (resp. quasi-coherent $\mathcal{O}_{X}$-modules) on the lisse-étale topos of $X[\mathrm{LMB}, 12.1]$. Let $\mathrm{D}(X)$ (resp. $\left.\mathrm{D}_{\mathrm{qc}}(X)\right)$ denote the unbounded derived category of $\operatorname{Mod}(X)$ (resp. the full subcategory of $\mathrm{D}(X)$ with cohomology in $\mathrm{Q} C o h(X))$. Superscripts such as,,$+- \geq n$, and $b$ decorating $\mathrm{D}(X)$ and $\mathrm{D}_{\mathrm{qc}}(X)$ are to be interpreted as usual. 
If $X$ is a Deligne-Mumford stack (e.g., a scheme or an algebraic space), then there is an associated small étale topos which we denote as $X_{\text {ét }}$. There is a natural morphism of ringed topoi res $_{X}: X_{\text {lis-ét }} \rightarrow X_{\text {ét. }}$ Let $\operatorname{Mod}\left(X_{\text {ét }}\right)\left(\operatorname{resp}\right.$. QCoh $\left.\left(X_{\text {ét }}\right)\right)$ denote

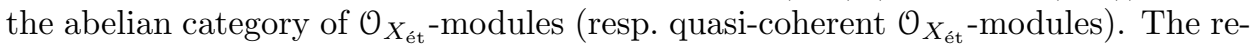
striction of $\left(\operatorname{res}_{X}\right)_{*}: \operatorname{Mod}(X) \rightarrow \operatorname{Mod}\left(X_{\text {ét }}\right)$ to $\mathrm{QCoh}(X)$ is fully faithful with essential image $\mathrm{QCoh}\left(X_{\text {ét }}\right)$ LMB, Prop. 13.2.3]. Let $\mathrm{D}_{\mathrm{qc}}\left(X_{\text {ét }}\right)$ denote the triangulated category $\mathrm{D}_{\mathrm{Q} \operatorname{Coh}\left(X_{\text {ét }}\right)}\left(\operatorname{Mod}\left(X_{\text {ét }}\right)\right)$. Then the natural functor $\mathrm{R}\left(\operatorname{res}_{X}\right)_{*}: \mathrm{D}_{\mathrm{qc}}(X) \rightarrow$ $\mathrm{D}_{\mathrm{qc}}\left(X_{\text {ét }}\right)$ is an equivalence of categories [LMB, Prop. 12.10.1]. If $X$ is a scheme, then the corresponding statement for the Zariski topos also holds [LMB, Lem.13.1.5].

1.1. Hypercoverings and simplicial sites. We now recall the relationship between the unbounded derived categories of quasi-coherent sheaves on an algebraic stack and those on a smooth hypercovering (i.e., cohomological descent). Our approach follows [Ols07] and [LO08]. Let $X$ be an algebraic stack and let $p_{\bullet}: U_{\bullet} \rightarrow X$ be a smooth hypercovering by algebraic spaces. Typically, we will take $U_{\bullet}$ to be the 0 -coskeleton associated to a smooth covering $p_{0}: U_{0} \rightarrow X$, where $U_{0}$ is an algebraic space. In plainer language, $U_{n}$ is the $(n+1)$ th fiber product of $U_{0}$ over $X$ and the simplicial structure (i.e., face and degeneracy maps) come from the various projections and diagonals between the $U_{n}$ as $n$ varies.

The simplicial algebraic space $U_{\bullet}$ gives rise to two semi-simplicial topoi: $U_{\bullet}^{+}$,lis-ét and $U_{\bullet \text {,ét }}^{+}$. The semi-simplicial topos $U_{\bullet}^{+}$ét is formed as follows: for each integer $n \geq 0$ there is the étale topos $U_{n \text {,ét }}$ and for each injective map $\delta:[n]=\{0, \ldots, n\} \rightarrow$

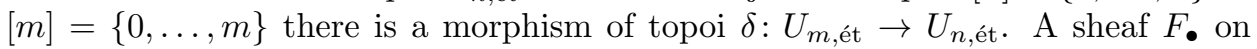

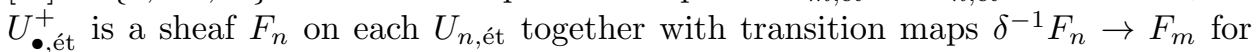
each injective map $\delta:[n] \rightarrow[m]$ that are compatible with composition; the sheaf

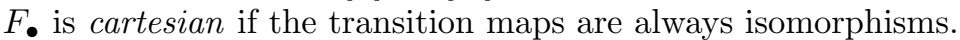

The topos $U_{\bullet}^{+}$,ét is naturally ringed by the flat sheaf $\mathcal{O}_{U_{\bullet} \text { ét }}^{+}$. Here flat means that the transition maps $\delta^{-1} \mathcal{O}_{U_{n}}$,ét $\rightarrow \mathcal{O}_{U_{m} \text {,ét }}$ are flat. Let $\operatorname{Mod}\left(U_{\bullet \text {,ét }}^{+}\right)$denote the associated category of modules and $\operatorname{Mod}_{\text {cart }}\left(U_{\bullet}^{+}\right.$,ét $)$the subcategory of cartesian

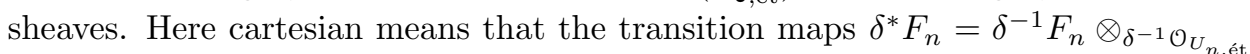
$\mathcal{O}_{U_{m, \text { ét }}} \rightarrow F_{m}$ are isomorphisms.

An $\mathcal{O}_{U_{\bullet} \text {,ét }}^{+}$-module is quasi-coherent if it is cartesian and its restriction to each

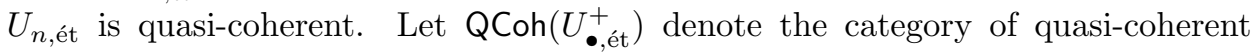
sheaves. Let $\mathrm{D}\left(U_{\bullet}^{+}\right.$,ét $)$be the unbounded derived category of $\operatorname{Mod}\left(U_{\bullet}^{+}\right.$,ét $)$, let $\mathrm{D}_{\text {cart }}\left(U_{\bullet}^{+}\right.$,ét $)$ denote the subcategory whose objects have cartesian cohomology sheaves and let $\mathrm{D}_{\mathrm{qc}}\left(U_{\bullet}^{+}\right.$ét $)$denote the subcategory with quasi-coherent cohomology sheaves. The semi-simplicial topos $U_{\bullet}^{+}$, lis-ét and its various module categories are defined similarly.

Thus, there are natural morphisms of ringed topoi:

$$
X_{\text {lis-ét }} \stackrel{p_{\bullet, \text { lis-ét }}^{+}}{\longleftarrow} U_{\bullet, \text { lis-ét }}^{+} \stackrel{\operatorname{res}_{\bullet}}{\longrightarrow} U_{\bullet, \text { ét }}^{+} \text {. }
$$

One way phrasing smooth descent of quasi-coherent sheaves is that these morphisms of topoi induce equivalences of abelian categories:

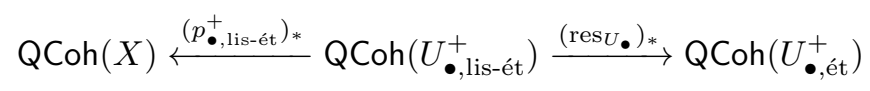

In [LO08, Ex. 2.2.5], it is shown that this can be improved to unbounded cohomological descent, that is, these morphism of topoi induce equivalences of triangulated categories:

$$
\mathrm{D}_{\mathrm{qc}}(X) \stackrel{\mathrm{R}\left(p_{\bullet, \text { lis-ét }}^{+}\right)_{*}}{\longleftarrow} \mathrm{D}_{\mathrm{qc}}\left(U_{\bullet, \text { lis-ét }}^{+} \stackrel{\left.\mathrm{R}\left(\operatorname{res}_{\bullet}\right)_{*}\right)}{\longrightarrow} \mathrm{D}_{\mathrm{qc}}\left(U_{\bullet, \text { ét }}^{+}\right) .\right.
$$

The morphisms $p_{\bullet}^{+}$,lis-ét and $\operatorname{res}_{U}$. have left exact inverse image functors. 
1.2. Operations in unbounded categories of modules. We now record for future reference some useful formulas [KS06, $\S \S 18.4$ and 18.6]. If $\mathcal{M}$ and $\mathcal{N} \in \mathrm{D}(X)$, then there is

$$
\begin{array}{rlrl}
\mathcal{M} \otimes_{\mathcal{O}_{X}}^{L} \mathcal{N} & \in \mathrm{D}(X) & \text { (the derived tensor product) } \\
\mathrm{RH}_{\mathcal{H} o m_{\mathcal{O}_{X}}(\mathcal{M}, \mathcal{N})} \in \mathrm{D}(X) & \text { (the derived sheaf Hom functor) } \\
\operatorname{RHom}_{\mathcal{O}_{X}}(\mathcal{M}, \mathcal{N}) & \in \mathrm{D}(\mathrm{Ab}) & \text { (the derived global Hom functor) }
\end{array}
$$

If in addition $\mathcal{P} \in \mathrm{D}(X)$, then we have a functorial isomorphism:

$$
\operatorname{Hom}_{\mathcal{O}_{X}}\left(\mathcal{M} \otimes_{\mathcal{O}_{X}}^{L} \mathcal{N}, \mathcal{P}\right) \cong \operatorname{Hom}_{\mathcal{O}_{X}}\left(\mathcal{M}, \operatorname{RHom}_{\mathcal{O}_{X}}(\mathcal{N}, \mathcal{P})\right),
$$

as well as a functorial quasi-isomorphism:

$$
\mathrm{RH} \operatorname{Hom}_{\mathcal{O}_{X}}\left(\mathcal{M} \otimes_{\mathcal{O}_{X}}^{\mathrm{L}} \mathcal{N}, \mathcal{P}\right) \simeq \mathrm{RH} \mathcal{H o m}_{\mathcal{O}_{X}}\left(\mathcal{M}, \mathrm{RH} \operatorname{Hom}_{\mathcal{O}_{X}}(\mathcal{N}, \mathcal{P})\right) .
$$

Letting $\mathrm{R} \Gamma(X,-)=\operatorname{RHom}_{\mathcal{O}_{X}}\left(\mathcal{O}_{X},-\right)$, there is also a natural quasi-isomorphism:

$$
\operatorname{RHom}_{\mathcal{O}_{X}}(\mathcal{M}, \mathcal{N}) \simeq \operatorname{R\Gamma RHom} \mathcal{O}_{X}(\mathcal{M}, \mathcal{N}) \text {. }
$$

If $\mathcal{M}$ and $\mathcal{N}$ belong to $\mathrm{D}_{\mathrm{qc}}(X)$, then $\mathcal{M} \otimes_{\mathcal{O}_{X}}^{\mathrm{L}} \mathcal{N} \in \mathrm{D}_{\mathrm{qc}}(X)$. Indeed, using homotopy colimits and triangles we reduce to the case where $\mathcal{M}$ and $\mathcal{N}$ are quasi-coherent sheaves. Then it follows from the definition of quasi-coherence [LMB, Déf. 13.2.2]. Since the category $\mathrm{D}_{\mathrm{qc}}(X)$ is well generated [HNR14, Thm. B.1] and the functor $-\otimes_{\mathcal{O}_{X}}^{\llcorner} \mathcal{M}: \mathrm{D}_{\mathrm{qc}}(X) \rightarrow \mathrm{D}_{\mathrm{qc}}(X)$ preserves small coproducts, it admits a right adjoint Nee01b, Thm. 8.4.4]

$$
\mathrm{RH}_{\mathcal{H} o m_{X}^{\mathrm{qc}}}^{\mathrm{qc}}(\mathcal{M},-): \mathrm{D}_{\mathrm{qc}}(X) \rightarrow \mathrm{D}_{\mathrm{qc}}(X)
$$

In fact, if

$$
Q_{X}: \mathrm{D}(X) \rightarrow \mathrm{D}_{\mathrm{qc}}(X)
$$

is the right adjoint to the inclusion $\mathrm{D}_{\mathrm{qc}}(X) \subseteq \mathrm{D}(X)$, which exists for the same reasons as above, then

$$
\mathrm{RH}^{\mathrm{H} o m_{\mathcal{O}_{X}}^{\mathrm{qc}}}(\mathcal{M},-) \simeq Q_{X}\left(\mathrm{RH}_{\mathcal{H}} m_{\mathcal{O}_{X}}(\mathcal{M},-)\right) .
$$

Note that while the formation of $\mathrm{RH} \operatorname{Hom}_{\mathcal{O}_{X}}(\mathcal{M},-)$ is smooth local on $X$, this is not true in general for $\mathrm{R} \mathcal{H}$ om $_{\mathcal{O}_{X}}^{\mathrm{qc}}(\mathcal{M},-)$. It is true, however, if $\mathcal{M}$ is perfect (Lemma 4.322).

1.3. Direct and inverse image. For a morphism of algebraic stacks $f: X \rightarrow Y$, the induced morphism of ringed topoi $f_{\text {lis-ét }}: X_{\text {lis-ét }} \rightarrow Y_{\text {lis-ét does not necessarily }}$ have a left exact inverse image functor Beh03, 5.3.12]. Thus the construction of the correct derived functors of $f^{*}: \mathrm{Q} \operatorname{Coh}(Y) \rightarrow \mathrm{Q} \operatorname{Coh}(X)$ is somewhat subtle. There are currently two approaches to constructing these functors. The first, due to M. Olsson Ols07] and Y. Laszlo and M. Olsson [LO08, uses cohomological descent. The other approach appears in the Stacks Project [Stacks, Tag 07BD]. In this article, we will employ the approach of Olsson and Laszlo-Olsson, which we now briefly recall.

Let $f: X \rightarrow Y$ be a morphism of algebraic stacks. Let $q: V \rightarrow Y$ be a smooth surjection from an algebraic space. Let $U \rightarrow X \times_{Y} V$ be another smooth surjection from an algebraic space. Let $\tilde{f}: U \rightarrow V$ be the resulting morphism of algebraic spaces and let $p: U \rightarrow X$ be the resulting smooth covering. By 11.1$)$, there is an induced 2-commutative diagram of ringed topoi:

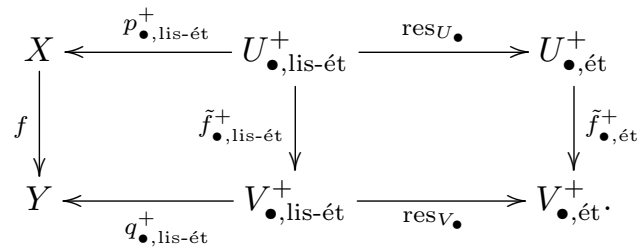


The 2-commutativity of the diagram above induces natural transformations:

$$
\begin{aligned}
& \mathrm{R}\left(f_{\text {lis-ét }}\right)_{*} \Rightarrow \mathrm{R}\left(q_{\bullet, \text { lis-étt }}^{+}\right)_{*} \mathrm{R}\left(\tilde{f}_{\bullet, \text { lis-ét }}^{+}\right)_{*} \mathrm{~L}\left(p_{\bullet, \text { lis-ét }}^{+}\right)^{*} \text { and } \\
& \mathrm{R}\left(\tilde{f}_{\bullet}^{+} \text {,ét }\right)_{*} \Rightarrow \mathrm{R}\left(\operatorname{res}_{V_{\bullet}}\right)_{*} \mathrm{R}\left(\tilde{f}_{\bullet}^{+} \text {,lis-ét }\right)_{*} \mathrm{~L}\left(\operatorname{res}_{U_{\bullet}}\right)^{*},
\end{aligned}
$$

which are natural isomorphisms for those complexes with quasi-coherent cohomology that are sent to complexes with quasi-coherent cohomology by $\mathrm{R}\left(\tilde{f}_{\bullet}^{+}{ }_{\text {,lis-ét }}\right)_{*}$ or $\mathrm{R}\left(\tilde{f}_{\bullet, \text { ét }}^{+}\right)_{*}$.

Remark 1.1. Note, however, that if $f: X \rightarrow Y$ is not representable, then $\mathrm{R}\left(f_{\text {lis-ét }}\right)_{*}$ does not, in general, send $\mathrm{D}_{\mathrm{qc}}(X)$ to $\mathrm{D}_{\mathrm{qc}}(Y)$ - even if $f$ is proper and étale and $X$ and $Y$ are smooth Deligne-Mumford stacks [Stacks, 07DC]. The problem is that quasi-compact and quasi-separated morphisms of algebraic stacks can have unbounded cohomological dimension, which is in contrast to the case of schemes and algebraic spaces [Stacks, 073G]. In the next section we will clarify this with the concept of a concentrated morphism.

Another crucial observation here is that the morphism of topoi $\tilde{f}_{\bullet}^{+}$ét has a left exact inverse image functor. The general theory now gives rise to an unbounded derived functor $\mathrm{L}\left(\tilde{f}_{\bullet}^{+} \text {,ét }\right)^{*}: \mathrm{D}\left(V_{\bullet}^{+}\right.$,ét $) \rightarrow \mathrm{D}\left(U_{\bullet}^{+}\right.$,ét $)$, which is left adjoint to $\mathrm{R}\left(\tilde{f}_{\bullet}^{+}{ }_{\bullet \text { ét }}\right)_{*}: \mathrm{D}\left(U_{\bullet}^{+}\right.$ét $) \rightarrow \mathrm{D}\left(V_{\bullet}^{+}{ }_{\text {ét }}\right)$. The functor $\mathrm{L}\left(\tilde{f}_{\bullet}^{+}, \text {ét }\right)^{*}$ is easily verified to preserve small coproducts and complexes with quasi-coherent cohomology. Using the equivalences of 1.2 , we may now define a functor $\mathrm{L} f_{\mathrm{qc}}^{*}: \mathrm{D}_{\mathrm{qc}}(Y) \rightarrow \mathrm{D}_{\mathrm{qc}}(X)$ such that $\mathcal{H}^{0}\left(\mathrm{~L} f_{\mathrm{qc}}^{*} \mathcal{M}[0]\right) \cong f^{*} \mathcal{M}$, whenever $\mathcal{M} \in \mathrm{Q} \operatorname{Coh}(Y)$. It is readily verified that the functor $L f_{\text {qc }}^{*}$ is unique up to canonical natural isomorphism, that is, it does not depend on the choice of charts $U$ and $V$. If $f: X \rightarrow Y$ is flat, then for all integers $q$ and all $\mathcal{M} \in \mathrm{D}_{\mathrm{qc}}(Y)$ there is a natural isomorphism:

$$
f^{*} \mathcal{H}^{q}(\mathcal{M}) \cong \mathcal{H}^{q}\left(\mathrm{~L} f_{\mathrm{qc}}^{*} \mathcal{M}\right)
$$

Since the category $\mathrm{D}_{\mathrm{qc}}(Y)$ is well generated HNR14, Thm. B.1] and the functor $\mathrm{L} f_{\mathrm{qc}}^{*}$ preserves small coproducts, it admits a right adjoint Nee01b, Thm. 8.4.4]

$$
\mathrm{R}\left(f_{\mathrm{qc}}\right)_{*}: \mathrm{D}_{\mathrm{qc}}(X) \rightarrow \mathrm{D}_{\mathrm{qc}}(Y)
$$

The functor above is closely related to the functors we have already seen. Indeed, since $\mathrm{L} f_{\mathrm{qc}}^{*} \mathcal{O}_{Y}[0] \simeq \mathcal{O}_{X}[0]$, it follows that if $\mathcal{M} \in \mathrm{D}_{\mathrm{qc}}(X)$, then

$$
\mathrm{R} \Gamma\left(Y, \mathrm{R}\left(f_{\mathrm{qc}}\right)_{*} \mathcal{M}\right) \simeq \mathrm{R} \Gamma(X, \mathcal{M}) .
$$

We now describe $\mathrm{R}\left(f_{\mathrm{qc}}\right)_{*}$ locally. Let

$$
\mathcal{Q}_{V_{\bullet, \text { ét }}^{+}}: \mathrm{D}\left(V_{\bullet, \text { ét }}^{+}\right) \rightarrow \mathrm{D}_{\mathrm{qc}}\left(V_{\bullet, \text { ét }}^{+}\right)
$$

be a right adjoint to the natural inclusion functor $\mathrm{D}_{\mathrm{qc}}\left(V_{\bullet}^{+}\right.$,ét $) \rightarrow \mathrm{D}\left(V_{\bullet}^{+}\right.$,ét $\left.^{+}\right)$, which exists by Nee01b, Thm. 8.4.4]. A straightforward calculation, utilizing the equivalences 1.2 , induces a natural isomorphism of functors:

$$
\mathrm{R}\left(f_{\mathrm{qc}}\right)_{*} \simeq \mathrm{R}\left(q_{\bullet, \text { lis-ét }}^{+}\right)_{*} \mathrm{~L}\left(\operatorname{res}_{V_{\bullet}}\right)^{*} Q_{V_{\bullet}^{+}+\text {ét }} \mathrm{R}\left(\tilde{f}_{\bullet \text {,ét }}^{+}\right)_{*} \mathrm{R}\left(\operatorname{res}_{U_{\bullet}}\right)_{*} \mathrm{~L}\left(p_{\bullet, \text { lis-ét }}^{+}\right)^{*} .
$$

The following Lemma clarifies the situation somewhat.

Lemma 1.2. If $f: X \rightarrow Y$ is a morphism of algebraic stacks that is quasi-compact and quasi-separated, then the following hold.

(1) The restriction of $\mathrm{R}\left(f_{\text {lis-ét }}\right)_{*}$ to $\mathrm{D}_{\mathrm{qc}}^{+}(X)$ factors through $\mathrm{D}_{\mathrm{qc}}^{+}(Y)$.

(2) The restrictions of $\mathrm{R}\left(f_{\text {lis-ét }}\right)_{*}$ and $\mathrm{R}\left(f_{\mathrm{qc}}\right)_{*}$ to $\mathrm{D}_{\mathrm{qc}}^{+}(X)$ are isomorphic.

(3) For each integer $d$, the restriction of the functor $\mathrm{R}\left(f_{\mathrm{qc}}\right)_{*}$ to $\mathrm{D}_{\mathrm{qc}}^{[d, \infty)}(X)$ preserves direct limits (in particular, small coproducts). 
(4) Consider a 2-cartesian diagram of algebraic stacks:

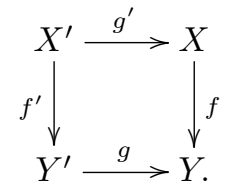

If $g$ is flat, then the base change transformation

$$
\mathrm{L} g_{\mathrm{qc}}^{*} \mathrm{R}\left(f_{\mathrm{qc}}\right)_{*} \Rightarrow \mathrm{R}\left(f_{\mathrm{qc}}^{\prime}\right)_{*} \mathrm{~L}\left(g^{\prime}\right)_{\mathrm{qc}}^{*}
$$

is an isomorphism upon restriction to $\mathrm{D}_{\mathrm{qc}}^{+}(X)$.

Proof. Claim (1) is Ols07, Lem. 6.20]. Claim (2) follows from cohomological descent (1.2), claim (1) and equations (1.7), (1.8), and (1.11).

For (3), by (2), we may replace $\mathrm{R}\left(f_{\mathrm{qc}}\right)_{*}$ by $\mathrm{R}\left(f_{\text {lis-ét }}\right)_{*}$. The hypercohomology spectral sequence:

$$
\mathrm{R}^{r}\left(f_{\text {lis-ét }}\right)_{*} \mathcal{H}^{s}(\mathcal{M}) \Rightarrow \mathrm{R}^{r+s}\left(f_{\text {lis-ét }}\right)_{*} \mathcal{M}
$$

now applies and it is thus sufficient to prove that the higher pushforwards

$$
\mathrm{R}^{r}\left(f_{\text {lis-ét }}\right)_{*}: \mathrm{QCoh}(X) \rightarrow \mathrm{QCoh}(Y)
$$

preserve direct limits for every integer $r \geq 0$. This is local on $Y$ for the smooth topology, so we may assume that $Y$ is an affine scheme. Thus it suffices to prove that the cohomology functors $H^{r}\left(X_{\text {lis-ét }},-\right): \mathrm{Q} \operatorname{Coh}(X) \rightarrow$ Ab preserve direct limits for every integer $r \geq 0$. Since $X$ is quasi-compact and quasi-separated, this is well-known (e.g., [Stacks, 0739]).

The base change transformation of (4) exists by functoriality of the adjoints. Applying (2) we may replace $\left(f_{\mathrm{qc}}\right)_{*}$ and $\left(f_{\mathrm{qc}}^{\prime}\right)_{*}$ by $\left(f_{\text {lis-ét }}\right)_{*}$ and $\left(f_{\text {lis-ét }}^{\prime}\right)_{*}$ respectively. The statement is now local on $Y$ and $Y^{\prime}$ for the smooth topology, so we may assume that both $Y$ and $Y^{\prime}$ are affine schemes. Small modifications to the argument of Stacks, 073K] complete the proof.

In $\sqrt{2}$ we describe a class of morphisms for which the conclusions of Lemma 1.2 remain valid in the unbounded derived category.

1.4. Comparison with definitions in derived algebraic geometry. We will now compare the derived category of quasi-coherent sheaves $\mathrm{D}_{\mathrm{qc}}(X)$ that we are using in this paper with derived categories of quasi-coherent sheaves as defined in derived algebraic geometry. This is not used in the remainder of the paper (but see Example 9.4).

First recall that if $\mathcal{A}$ is an abelian category, then the derived category $\mathrm{D}(\mathcal{A})$ is the homotopy category of a natural stable $\infty$-category $\mathcal{D}(\mathcal{A})[\mathrm{HA}$, Def. 1.3.5.8]. If in addition $\mathcal{C} \subseteq \mathcal{A}$ is a weak Serre subcategory, then we can consider the full $\infty$-subcategory $\mathcal{D}_{\mathcal{C}}(\mathcal{A})$ of objects with cohomology in $\mathcal{C}$ and this has homotopy category $\mathrm{D}_{\mathcal{C}}(\mathcal{A})$. We thus define $\mathcal{D}_{\mathrm{qc}}(X):=\mathcal{D}_{\mathrm{QCoh}(X)}(\operatorname{Mod}(X))$ which has homotopy category $\mathrm{D}_{\mathrm{qc}}(X)$. If $X$ is Deligne-Mumford, we also define $\mathcal{D}_{\mathrm{qc}}\left(X_{\text {ét }}\right):=$ $\mathcal{D}_{\mathrm{Q} \operatorname{Coh}\left(X_{\text {ét }}\right)}\left(\operatorname{Mod}\left(X_{\text {ét }}\right)\right)$ which is equivalent to $\mathcal{D}_{\mathrm{qc}}(X)$ [LMB, Prop. 12.10.1].

Proposition 1.3 (cf. [DG13, Rem. 1.2.3]). Let $X$ be an algebraic stack. Then

$$
\mathcal{D}_{\mathrm{qc}}(X)=\underbrace{}_{\text {Spec } A \rightarrow X} \mathcal{D}(\operatorname{Mod}(A))
$$

where the limit is taken in the $\infty$-category of $\infty$-categories and is over either $(i)$ the category of all affine schemes over $X$, (ii) the full subcategory of those smooth over $X$, or (iii) the subcategory of those smooth over $X$ with only smooth $X$-morphisms $\operatorname{Spec} A \rightarrow \operatorname{Spec} B$. 
We denote the limit of the right-hand side going over all morphisms by $Q \mathcal{C o h}(X)$. This is a stable $\infty$-category which is left- and right-complete, and has a t-structure with heart the abelian category $\mathrm{Q} C o h(X)$ SAG, Cor. 9.1.3.2, Rem. 9.1.3.3]. The $\infty$-category $Q \mathcal{C o h}(X)$ also makes sense for any contravariant functor $X$ from affine schemes to groupoids and can be adapted to variants in derived algebraic geometry. This is how derived categories of quasi-coherent sheaves usually are defined in derived algebraic geometry, cf. [BZFN10, §3.1], [DG13, §1.2], [SAG, §6.2.2] and GR17, Ch. 3, §1.1].

The category $\mathrm{D}_{\mathrm{qc}}(X)$ is left-complete HNR14, Thm. B.1]. We do not use this fact in the proof so we obtain an independent proof of the left-completeness of both $\mathrm{D}_{\mathrm{qc}}(X)$ and $\mathcal{D}_{\mathrm{qc}}(X)$.

Proof of Proposition 1.3. The limit restricted to smooth morphisms, or to smooth morphisms with smooth maps between them, is also equivalent to $2 \operatorname{Coh}(X)$ GR17, Ch. $3, \S 1.4 .2]$. Moreover, if $U \rightarrow X$ is a smooth presentation and $U_{\bullet}^{+}$is the corresponding semi-simplicial algebraic space, then restricting the limit to the diagram $U_{\bullet}^{+}$gives the same limit (use that $X$ is a stack and that $\Delta^{+} \subseteq \Delta$ is right cofinal, cf. [SAG, Prop. 6.2.3.1 and pf. of Prop. 9.1.3.1] or GR17, Ch. 3, Cor. 1.3.11]). We may also instead take the limit over the site $U_{\bullet \text {,ét }}^{+}$since $U_{\bullet}^{+}: \Delta^{+} \rightarrow U_{\bullet}^{+}$,ét is right cofinal.

We have a sequence of maps between $\infty$-categories

$$
\begin{aligned}
& \mathcal{D}_{\mathrm{qc}}(X) \stackrel{\alpha}{\longrightarrow} \mathcal{D}_{\mathrm{qc}}\left(U_{\bullet}^{+}, \text {ét }\right) \stackrel{\beta}{\longrightarrow} \lim _{V \in U_{\bullet}^{+}, \text {ét }} \mathcal{D}_{\mathrm{qc}}\left(U_{\bullet \text {,ét }}^{+} / V\right) \\
& \stackrel{\gamma}{\longrightarrow} \lim _{V \in U_{\bullet}^{+} \text {ét }} \mathcal{D}_{\mathrm{qc}}(V) \stackrel{\delta}{\longleftarrow} \operatorname{QCoh}(X) .
\end{aligned}
$$

That $\alpha$ is an equivalence follows by unbounded cohomological descent $(1.2)$. That $\beta$ is an equivalence follows from Lemma 1.6 applied to the semi-simplicial étale site $U_{\bullet \text {,ét }}^{+}$since having quasi-coherent cohomology can be verified locally. The map $\gamma$ comes from the morphism of topoi $\epsilon: U_{\bullet}^{+}$ét $/ V \rightarrow V_{\text {ét }}$. Since $\epsilon_{*}$ (restriction) is exact and $\epsilon^{*}$ is exact and fully faithful with essential image the cartesian modules, we obtain equivalences between cartesian modules and between derived categories of modules with cartesian cohomology sheaves, cf. [LMB, Prop. 12.10.1]. This shows that $\gamma$ is an equivalence. We saw that $\delta$ was an equivalence above.

Remark 1.4. The maps $\beta$ and $\gamma$ are also equivalences if we replace quasi-coherent cohomology with cartesian cohomology.

Remark 1.5. If in addition $X$ has affine diagonal, or is noetherian and affine-pointed, then the natural functor $\mathrm{D}^{+}(\mathrm{Q} \operatorname{Coh}(X)) \rightarrow \mathrm{D}_{\mathrm{qc}}^{+}(X)$ is an equivalence [HNR14, App. C]. It follows that $\mathcal{D}^{+}(\mathrm{Q} \operatorname{Coh}(X)) \rightarrow \mathcal{Q C o h}^{+}(X)$ is an equivalence, which can also be proven directly, cf. [DG13, Rem. 1.2.10] and GR17, Ch. 3, Prop. 2.4.3]. Note that $\mathrm{D}(\mathrm{Q} \operatorname{Coh}(X))$ and $\mathcal{D}(\mathrm{Q} \operatorname{Coh}(X))$ are not always left-complete, e.g., when $X=B \mathbb{G}_{a}$ in positive characteristic [Nee11. They are left-complete, and hence coincide with $\mathrm{D}_{\mathrm{qc}}(X)$ and $\mathcal{D}_{\mathrm{qc}}(X)$, respectively, when $\mathrm{D}_{\mathrm{qc}}(X)$ is compactly generated [HNR14, Thm. 1.2].

Lemma 1.6. Let $(\mathcal{T}, \mathcal{O})$ be a ringed topos and let $\left(\mathcal{T} / U,\left.\mathcal{O}\right|_{U}\right)$ denote the localized topos for any $U \in \mathcal{T}$. Then the assignment $U \mapsto \mathcal{D}\left(\operatorname{Mod}\left(\left.\mathcal{O}\right|_{U}\right)\right)$ is a sheaf, that is, there is a limit preserving functor

$$
\begin{aligned}
\mathcal{T}^{\circ} & \longrightarrow \mathrm{Cat}_{\infty} \\
U & \longmapsto \mathcal{D}\left(\operatorname{Mod}\left(\left.\mathcal{O}\right|_{U}\right)\right) .
\end{aligned}
$$


Proof. We identify a ringed topos with a $\mathbb{E}_{\infty}$-ringed $\infty$-topos by taking nerves. Such an $\infty$-topos is a 1-topos, that is, discrete, and $\mathcal{O}$ is discrete. We let $\mathcal{M}_{0} d_{\mathcal{O}}$ denote the $\infty$-category of $\mathcal{O}$-module spectra. Its heart is the category of usual modules $\operatorname{Mod}(\mathcal{O})$ SAG, Def. 2.1.0.1, Rem. 2.1.2.1]. By the universal property of derived categories, there is a functor $\mathcal{D}^{+}\left(\operatorname{Mod}\left(\left.\mathcal{O}\right|_{U}\right)\right) \rightarrow \operatorname{Mod}_{\left.\mathcal{O}\right|_{U}}$. This functor extends to a fully faithful functor $\mathcal{D}\left(\operatorname{Mod}\left(\left.\mathcal{O}\right|_{U}\right)\right) \rightarrow \operatorname{\mathcal {Mod}}_{\left.\mathcal{O}\right|_{U}}$ whose essential image is the full subcategory of hypercomplete objects [SAG, Cor. 2.1.2.3].

By SAG, Rem. 2.1.0.5], there is a limit-preserving functor $U \mapsto \mathcal{M o d}_{\left.\mathcal{O}\right|_{U}}$. Since being hypercomplete is a local property [HTT, Rem. 6.5.2.22], we obtain a limitpreserving functor $U \mapsto \mathcal{D}\left(\operatorname{Mod}\left(\left.\mathcal{O}\right|_{U}\right)\right)$.

Remark 1.7 (Deligne-Mumford stacks). If $X$ is a Deligne-Mumford stack, we can associate a spectral Deligne-Mumford stack $\mathfrak{X}$ to $X$. To $\mathfrak{X}$, one associates a stable $\infty$-category of quasi-coherent sheaves $2 \operatorname{Coh}(\mathfrak{X})$, a subcategory of $\mathcal{M} \operatorname{od}(\mathfrak{X})=$ $\mathcal{M} \operatorname{Cod}\left(\mathcal{O}_{X_{\text {ét }}}\right)$. It is equivalent to $\mathcal{L} \operatorname{Coh}(X)$ as defined above [SAG, Prop. 6.2.4.1], hence to $\mathcal{D}_{\mathrm{qc}}(X)$. This can also be seen directly as follows [SAG, Cor. 2.2.6.2]. The $\infty$-category $\mathcal{D}\left(\operatorname{Mod}\left(\mathcal{O}_{X_{\text {ét }}}\right)\right)$ can be identified with the full subcategory of hypercomplete objects of $\operatorname{Mod}(\mathfrak{X})[\mathrm{SAG}$, Cor. 2.1.2.3] and $\mathcal{Q} \operatorname{Coh}(\mathfrak{X})$ can be identified with the full subcategory of hypercomplete objects with quasi-coherent homotopy sheaves [SAG, Prop. 2.2.6.1]. That is, $\mathcal{D}_{\mathrm{qc}}\left(X_{\text {ét }}\right)=\mathcal{L} \operatorname{Coh}(\mathfrak{X})$.

\section{Concentrated morphisms of Algebraic Stacks}

A morphism of schemes $f: X \rightarrow Y$ is concentrated if it is quasi-compact and quasi-separated Lip09, §3.9]. Concentrated morphisms of schemes are natural to consider when working with unbounded derived categories of quasi-coherent sheaves. Indeed, if $f$ is concentrated, then

(1) $\mathrm{R}\left(f_{\mathrm{qc}}\right)_{*}$ coincides with the restriction of $\mathrm{R}\left(f_{\mathrm{Zar}}\right)_{*}$ to $\mathrm{D}_{\mathrm{qc}}(X)$,

(2) $\mathrm{R}\left(f_{\mathrm{qc}}\right)_{*}$ preserves small coproducts, and

(3) $\mathrm{R}\left(f_{\mathrm{qc}}\right)_{*}$ is compatible with flat base change on $Y$.

Here, as before, $\mathrm{R}\left(f_{\mathrm{qc}}\right)_{*}$ denotes the right adjoint to the unbounded derived functor $\mathrm{L} f_{\mathrm{qc}}^{*}: \mathrm{D}_{\mathrm{qc}}(Y) \rightarrow \mathrm{D}_{\mathrm{qc}}(X)$. In this section we isolate a class of morphisms of algebraic stacks, which we will also call concentrated, that enjoy the same properties.

Definition 2.1. Let $n \geq 0$ be an integer. A quasi-compact and quasi-separated morphism of algebraic stacks $f: X \rightarrow Y$ has cohomological dimension $\leq n$ if for all $i>n$ and all $\mathcal{M} \in \mathrm{Q} \operatorname{Coh}(X)$ we have that $\mathrm{R}^{i}\left(f_{\text {lis-ét }}\right)_{*} \mathcal{M}=0$ (by Lemma 1.2 2), this is equivalent to $\left.\mathrm{R}^{i}\left(f_{\mathrm{qc}}\right)_{*} \mathcal{M}=0\right)$.

The next result is inspired by [Alp13, Prop. 3.9], where similar results are proven in the context of cohomologically affine morphisms. Note, however, that cohomologically affine morphisms are not quite the same as morphisms of cohomological dimension $\leq 0$ Alp13, Rem. 3.5].

Lemma 2.2. Let $f: X \rightarrow Y$ be a 1-morphism of algebraic stacks that is quasicompact and quasi-separated. Let $n \geq 0$ be an integer.

(1) Let $\alpha: f \Rightarrow f^{\prime}$ be a 2-morphism. If $f$ has cohomological dimension $\leq n$, then so has $f^{\prime}$.

(2) Let $g: Z \rightarrow Y$ be a 1-morphism of algebraic stacks that is faithfully flat. If $f_{Z}: X \times_{Y} Z \rightarrow Z$ has cohomological dimension $\leq n$, then so has $f$.

(3) If $f$ is affine, then it has cohomological dimension $\leq 0$.

(4) Let $h: W \rightarrow X$ be a 1-morphism of algebraic stacks that is quasi-compact and quasi-separated and let $m \geq 0$ be an integer. If $f$ (resp. h) has cohomological dimension $\leq n$ (resp. $\leq m)$, then the composition $f \circ h: W \rightarrow Y$ has cohomological dimension $\leq m+n$. 
(5) Let $g: Z \rightarrow Y$ be a 1-morphism of algebraic stacks that is quasi-affine. If $f$ has cohomological dimension $\leq n$, then so has the 1-morphism $f_{Z}: X \times_{Y}$ $Z \rightarrow Z$.

(6) Let $g: Z \rightarrow Y$ be a 1-morphism of algebraic stacks. If $f$ has cohomological dimension $\leq n$ and $Y$ has quasi-affine diagonal, then the 1-morphism $f_{Z}: X \times_{Y} Z \rightarrow Z$ has cohomological dimension $\leq n$.

Proof. The claim (1) is trivial. To address the claim (2) we note that higher pushforwards commute with flat base change (Lemma 1.2 4) ). As faithfully flat morphisms are conservative, the morphism $f$ has cohomological dimension $\leq n$. The claim (3) follows trivially from (2). The claim (4) follows from the Leray spectral sequence.

We now address the claim (5). Denote the pullback of $g$ by $f$ as $g_{X}: Z_{X} \rightarrow X$ and throughout we fix $M \in \mathrm{Q} \operatorname{Coh}\left(Z_{X}\right)$. We first assume that the morphism $g$ is a quasicompact open immersion. In this situation the adjunction $\left(g_{X}\right)_{\mathrm{qc}}^{*}\left(\left(g_{X}\right)_{\text {lis-ét }}\right)_{*} M \rightarrow$ $M$ is an isomorphism. For $i \geq 0$ we deduce that there are isomorphisms in $\mathrm{QCoh}(Z)$ :

$$
\mathrm{R}^{i}\left(\left(f_{Z}\right)_{\text {lis-ét }}\right)_{*}\left(\left(g_{X}\right)_{\mathrm{qc}}^{*}\left(\left(g_{X}\right)_{\text {lis-ét }}\right)_{*} M\right) \rightarrow \mathrm{R}^{i}\left(\left(f_{Z}\right)_{\text {lis-ét }}\right)_{*} M .
$$

Since higher pushforward commute with flat base change, we deduce that for all $i \geq 0$ there are isomorphisms:

$$
g^{*} \mathrm{R}^{i}\left(f_{\text {lis-ét }}\right)_{*}\left(\left(\left(g_{X}\right)_{\text {lis-ét }}\right)_{*} M\right) \rightarrow \mathrm{R}^{i}\left(\left(f_{Z}\right)_{\text {lis-ét }}\right)_{*} M .
$$

Since $\left(\left(g_{X}\right)_{\text {lis-ét }}\right)_{*} M \in \mathrm{Q} \operatorname{Coh}(X)$, it follows that $f_{Z}: X_{Z} \rightarrow Z$ has cohomological dimension $\leq n$. Next assume that the morphism $g$ is affine. Then the morphism $g_{X}$ is also affine and so, by (3), both morphisms have cohomological dimension $\leq 0$. By (4) we conclude that the composition $f \circ g_{X}: Z_{X} \rightarrow Y$ has cohomological dimension $\leq n$. But we have a 2-isomorphism $f \circ g_{X} \Rightarrow g \circ f_{Z}$ and so by (1) the morphism $g \circ f_{Z}$ has cohomological dimension $\leq n$. By the Leray spectral sequence, however, we see that there is an isomorphism for all $i \geq 0$ :

$$
\left(g_{\text {lis-ét }}\right)_{*} \mathrm{R}^{i}\left(\left(f_{Z}\right)_{\text {lis-ét }}\right)_{*} M \rightarrow \mathrm{R}^{i}\left(\left(g \circ f_{Z}\right)_{\text {lis-ét }}\right)_{*} M .
$$

Since the morphism $g$ is affine, the functor $g_{*}$ is faithful; thus we conclude that the morphism $f_{Z}$ has cohomological dimension $\leq n$. In general, a quasi-affine morphism $g: Z \rightarrow Y$ factors as $Z \stackrel{j}{\rightarrow} \bar{Z} \stackrel{\bar{g}}{\rightarrow} Y$, where the morphism $j$ is a quasi-compact open immersion and the morphism $\bar{g}$ is affine. Combining the above completes the proof of (5).

To prove the claim (6) we observe that by $(2)$ the statement is smooth local on $Z$ - thus we are free to assume that $Z$ is an affine scheme. Since the diagonal of the stack $Y$ is quasi-affine, the morphism $g: Z \rightarrow Y$ is quasi-affine. An application of (5) now gives the claim.

We wish to point out that Lemma 2.2 6 is false if $Y$ does not have affine stabilizers [HR15, Rem. 1.6].

Definition 2.3. A quasi-compact and quasi-separated morphism $f: X \rightarrow Y$ of algebraic stacks has finite cohomological dimension if there exists an integer $n \geq 0$ such that the morphism $f$ has cohomological dimension $\leq n$.

Morphisms of quasi-compact and quasi-separated algebraic spaces have finite cohomological dimension Stacks, 073G]. V. Drinfeld and D. Gaitsgory DG13, Thm. 1.4.2, §2] have shown that a morphism of quasi-compact and quasi-separated algebraic stacks $f: X \rightarrow Y$ has finite cohomological dimension if $Y$ is a $\mathbb{Q}$-stack and $f$ has affine stabilizers and finitely presented inertia. This result is refined and generalized in [HR15]: the condition on inertia is not required and in positive characteristic $f$ has finite cohomological dimension exactly when $f$ has linearly reductive stabilizers. 
Definition 2.4. A morphism of algebraic stacks $f: X \rightarrow Y$ is concentrated if it is quasi-compact, quasi-separated, and for every quasi-compact and quasi-separated algebraic stack $Z$ and every morphism $g: Z \rightarrow Y$, the pulled back morphism $f_{Z}: X_{Z} \rightarrow Z$ has finite cohomological dimension.

By the result of Drinfeld and Gaitsgory, a quasi-compact and quasi-separated morphism of algebraic stacks $f: X \rightarrow Y$ is concentrated if $Y$ is a $\mathbb{Q}$-stack and $f$ has affine stabilizers.

The next result is immediate from Lemma 2.2

Lemma 2.5. Let $f: X \rightarrow Y$ be a 1-morphism of algebraic stacks that is quasicompact and quasi-separated.

(1) If $f$ is concentrated, then it remains so after base change.

(2) Let $g: Z \rightarrow Y$ be a 1-morphism that is faithfully flat. If $f_{Z}: X \times_{Y} Z \rightarrow Z$ is concentrated, then so is $f$.

(3) If $f$ is representable, then it is concentrated.

(4) Let $h: Y \rightarrow W$ be a 1-morphism that is concentrated. Then the composition $h \circ f: X \rightarrow W$ is concentrated if and only if $f$ is concentrated.

(5) Assume that $Y$ is quasi-compact with quasi-affine diagonal. Then $f$ is concentrated if and only if it has finite cohomological dimension.

The main result of this section is the following Theorem that refines Lemma 1.2 .

Theorem 2.6. Let $f: X \rightarrow Y$ be a concentrated 1-morphism of algebraic stacks

(1) If $Y$ is quasi-compact and quasi-separated, then there is an integer $n$ such that the natural morphism

$$
\tau^{\geq j} \mathrm{R}\left(f_{\mathrm{qc}}\right)_{*} \mathcal{M} \rightarrow \tau^{\geq j} \mathrm{R}\left(f_{\mathrm{qc}}\right)_{*}\left(\tau^{\geq j-n} \mathcal{M}\right)
$$

is a quasi-isomorphism for every integer $j$ and $\mathcal{M} \in \mathrm{D}_{\mathrm{qc}}(X)$.

(2) The restriction of $\mathrm{R}\left(f_{\text {lis-ét }}\right)_{*}$ to $\mathrm{D}_{\mathrm{qc}}(X)$ coincides with $\mathrm{R}\left(f_{\mathrm{qc}}\right)_{*}$.

(3) The functor $\mathrm{R}\left(f_{\mathrm{qc}}\right)_{*}$ preserves small coproducts.

(4) If $g: Y^{\prime} \rightarrow Y$ is a flat morphism of algebraic stacks, then the 2-cartesian square:

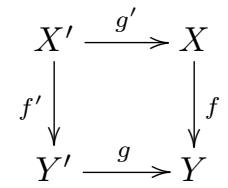

induces a natural quasi-isomorphism for every $\mathcal{M} \in \mathrm{D}_{\mathrm{qc}}(X)$ :

$$
\mathrm{L} g_{\mathrm{qc}}^{*} \mathrm{R}\left(f_{\mathrm{qc}}\right)_{*} \mathcal{M} \simeq \mathrm{R}\left(f_{\mathrm{qc}}^{\prime}\right)_{*} \mathrm{~L} g_{\mathrm{qc}}^{\prime *} \mathcal{M}
$$

Proof. For (2), choose a diagram as in (1.6). By the natural transformations (1.7), 1.8), and 1.11), it is sufficient to prove that the restriction of $\mathrm{R}\left(\tilde{f}_{\bullet}^{+} \text {,ét }\right)_{*}$ to $\mathrm{D}_{\mathrm{qc}}\left(U_{\bullet \text {,ét }}^{+}\right)$ factors through $\mathrm{D}_{\mathrm{qc}}\left(V_{\bullet}^{+}\right.$,ét $)$. This can be verified smooth locally on $Y$, so we may assume that $Y$ is quasi-compact and quasi-separated and $f$ has cohomological dimension $\leq n$ for some integer $n$. In particular, $\mathrm{R}^{i}\left(\tilde{f}_{\bullet}^{+} \text {ét }\right)_{*} \mathcal{M}=0$ for every $i>n$ and $\mathcal{M} \in \mathrm{QCoh}\left(U_{\bullet \text {,ét }}^{+}\right)$. By [LO08, Lem. 2.1.10], for every $\mathcal{M} \in \mathrm{D}_{\mathrm{qc}}\left(U_{\bullet}^{+}\right.$,ét $)$and integer $j$ the natural morphism:

$$
\tau^{\geq j} \mathrm{R}\left(\tilde{f}_{\bullet, \text { ét }}^{+}\right)_{*} \mathcal{M} \rightarrow \tau^{\geq j} \mathrm{R}\left(\tilde{f}_{\bullet, \text { ét }}^{+}\right)_{*}\left(\tau^{\geq j-n} \mathcal{M}\right)
$$

is an isomorphism. By Lemma 1.2 2 the result follows. Note that the equation (2.1) now proves (1). Finally, the claims (3) and (4) follow from (1) and the corresponding results for the bounded below category in Lemma 1.2 
Corollary 2.7. If $f: X \rightarrow Y$ is an affine morphism of algebraic stacks, then there is a natural equivalence of triangulated categories

$$
\bar{f}^{*}: \mathrm{D}_{\mathrm{qc}}\left(Y_{\text {lis-ét }}, f_{*} \mathcal{O}_{X}\right) \rightarrow \mathrm{D}_{\mathrm{qc}}(X) \text {. }
$$

Proof. Pick a diagram as in 1.6. Since $f$ is an affine morphism, we may assume that $U_{i}=X \times_{Y} V_{i}$. The morphism of ringed topoi $\tilde{f}_{\bullet \text {,ét }}^{+}: U_{\bullet}^{+}$,ét $\rightarrow V_{\bullet}^{+}$,ét factors as:

$$
\left(U_{\bullet, \text { ét }}^{+}, \mathcal{O}_{U_{\bullet, \text { ét }}^{+}}\right) \stackrel{g}{\rightarrow}\left(V_{\bullet, \text { ét }}^{+},\left(\tilde{f}_{\bullet, \text { ét }}^{+}\right)_{*} \mathcal{O}_{U_{\bullet}^{+}, \text {ét }}\right) \stackrel{k}{\rightarrow}\left(V_{\bullet, \text { ét }}^{+}, \mathcal{O}_{V_{\bullet}^{+}}^{+}\right) .
$$

We claim that $g^{-1}\left(\tilde{f}_{\bullet}^{+}, \text {ét }\right)_{*} \mathcal{O}_{U_{\bullet}^{+} \text {ét }} \rightarrow \mathcal{O}_{U_{\bullet}^{+} \text {ét }}$ is flat. It is sufficient to verify this upon restriction to each $\left(U_{i}\right)_{\text {ét }}$ and then work étale-locally; thus, we may assume that $f$ is a morphism of affine schemes $\operatorname{Spec} B \rightarrow \operatorname{Spec} A$. Hence, it suffices to prove that the induced morphism of ringed sites $\bar{f}:\left((\operatorname{Spec} B)_{\text {ét }}, \mathcal{O}_{\mathrm{Spec}} B_{\text {ét }}\right) \rightarrow$ $\left((\operatorname{Spec} A)_{\text {ét }},\left(f_{\text {ét }}\right)_{*} \mathcal{O}_{\text {Spec } B}\right)$ is flat. This can be verified at geometric points, so let $\mathfrak{p}$ be a prime ideal of $B$. We must prove that if $\mathfrak{q}=f(\mathfrak{p})$, then the induced ring homomorphism $B \otimes_{A} A_{\mathfrak{q}}^{\text {sh }} \rightarrow B_{\mathfrak{p}}^{\text {sh }}$ is flat, where sh denotes the strict henselization at the relevant prime ideal. Since $\operatorname{Spec}\left(B \otimes_{A} A_{\mathfrak{q}}^{\text {sh }}\right)$ has flat diagonal over $\operatorname{Spec} B$ and $\operatorname{Spec} B_{\mathfrak{p}}^{\text {sh }}$ is a flat $\operatorname{Spec} B$-scheme, the assertion is clear and the claim is proved.

It follows that $g^{*}: \operatorname{Mod}\left(V_{\bullet \text {,ét }}^{+},\left(\tilde{f}_{\bullet \text {,ét }}^{+}\right)_{*} \mathcal{O}_{U_{\bullet}^{+} \text {ét }}\right) \rightarrow \operatorname{Mod}\left(U_{\bullet \text {,ét }}^{+}, \mathcal{O}_{U_{\bullet}^{+}}{ }_{\bullet}\right)$ is exact. Moreover since $f$ is affine, it has cohomological dimension $\leq 0$ (Lemma 2.2 (3)) and is concentrated. In particular, the restriction of $\mathrm{Rg}_{*}$ to $\mathrm{D}_{\mathrm{qc}}\left(U_{\bullet \text {,ét }}^{+}, \mathcal{O}_{U_{\bullet}^{+} \text {,ét }}\right)$ factors through

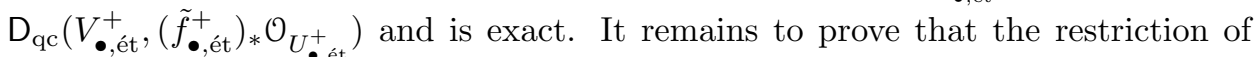
the adjunctions $\mathrm{Id} \stackrel{\mathrm{R}}{\Rightarrow} g_{*} \mathrm{~L} g^{*}$ and $\mathrm{L} g^{*} \mathrm{R} g_{*} \Rightarrow \mathrm{Id}$ to complexes with quasi-coherent cohomology sheaves are isomorphisms. By the exactness, it is sufficient to prove that the restrictions of the underived adjunctions Id $\Rightarrow g_{*} g^{*}$ and $g^{*} g_{*} \Rightarrow$ Id to quasi-coherent modules are isomorphisms. Unwinding the definitions, this is just the assertion that $\mathrm{Q} \operatorname{Coh}(X) \cong \mathrm{Q} \operatorname{Coh}\left(Y_{\text {lis-ét }}, f_{*} \mathcal{O}_{X}\right)$. By smooth descent, we may reduce to the situation where $X$ and $Y$ are affine schemes. The result now follows from [EGA, §II.1.4].

Corollary 2.8. Let $f: X \rightarrow Y$ be a quasi-affine morphism of algebraic stacks and let $M \in \mathrm{D}_{\mathrm{qc}}(X)$. If $\mathrm{R}\left(f_{\mathrm{qc}}\right)_{*} M \simeq 0$, then $M \simeq 0$, that is, $\mathrm{R}\left(f_{\mathrm{qc}}\right)_{*}$ is conservative.

Proof. We may factor $f$ as $X \stackrel{j}{\rightarrow} X^{\prime} \stackrel{f^{\prime}}{\rightarrow} Y$, where $j$ is a quasi-compact open immersion and $f^{\prime}$ is an affine morphism. By Corollary 2.7, the result is true for $f^{\prime}$. Hence, we are reduced to the situation where $f$ is a quasi-compact open immersion. In this case, however, $\Delta_{X / Y}: X \rightarrow X \times_{Y} X$ is an isomorphism. By Theorem 2.6 (4) $\mathrm{L} f_{\mathrm{qc}}^{*} \mathrm{R}\left(f_{\mathrm{qc}}\right)_{*} M \simeq M$ and the result follows.

\section{TRiangulated CATEgories}

In this section we will recall some results on triangulated categories that may not be familiar to everyone. For excellent and comprehensive treatments of these topics see [Nee92b] and [Tho97, §2]. In particular, we will recall thick and localizing triangulated subcategories. This leads to the concept of compact objects and Thomason's localization theorem.

Throughout this section, let $\mathcal{S}$ be a triangulated category with shift operator $\Sigma$.

A functor $F: \mathcal{S} \rightarrow \mathcal{S}^{\prime}$ between triangulated categories is triangulated if $F$ sends triangles to triangles and is compatible with shifts. We say that a full subcategory $\mathcal{R}$ of $\mathcal{S}$ is triangulated if the category $\mathcal{R}$ is triangulated and the inclusion functor $\mathcal{R} \rightarrow \mathcal{S}$ is triangulated. A subcategory $\mathcal{R} \subseteq \mathcal{S}$ is thick (also known as épaisse or saturated) if it is full, triangulated, and every $\mathcal{S}$-direct summand of every $r \in \mathcal{R}$ belongs to $\mathcal{R}$. 
Example 3.1. For a triangulated functor $F: \mathcal{S} \rightarrow \mathcal{S}^{\prime}$ we denote by ker $F$ the full triangulated subcategory consisting of those $x \in \mathcal{S}$ such that $F(x) \simeq 0$. The subcategory ker $F \subseteq \mathcal{S}$ is thick.

Example 3.2. Given triangulated subcategories $\mathcal{R}_{1} \subseteq \mathcal{R}_{2} \subseteq \mathcal{S}$ such that $\mathcal{R}_{1}$ is a thick subcategory of $\mathcal{S}$, then $\mathcal{R}_{1}$ is a thick subcategory $\mathcal{R}_{2}$.

Kernels of triangulated functors produce essentially all thick subcategories Tho97. §1.3]. Indeed, for every thick subcategory $\mathcal{R} \subseteq \mathcal{S}$ there is a quotient functor $Q: \mathcal{S} \rightarrow \mathcal{S} / \mathcal{R}$ such that $\mathcal{R} \cong \operatorname{ker} Q, Q$ is essentially surjective, and satisfies a universal property [Nee01b, Thm. 2.1.8].

For a class $R \subseteq \mathcal{S}$ the thick closure of $R$ is the smallest thick subcategory $\mathcal{R} \subseteq \mathcal{S}$ containing $R$. A subcategory $\mathcal{R} \subseteq \mathcal{S}$ is dense if it is full, triangulated, and its thick closure coincides with $\mathcal{S}$.

If the triangulated category $\mathcal{S}$ is essentially small, then there is a notion of $\mathrm{K}_{0}(\mathcal{S})$ : it is the free abelian group on the set of isomorphism classes of objects in $\mathcal{S}$ modulo the relation that given an $\mathcal{S}$-triangle $s_{1} \rightarrow s_{2} \rightarrow s_{3}$, then $\left[s_{2}\right]=\left[s_{1}\right]+\left[s_{3}\right]$. It is easy to see that for $s, t \in \mathcal{S}$ then $[s \oplus t]=[s]+[t]$ and $[s]=-[\Sigma s]$. Also, for every $\sigma \in \mathrm{K}_{0}(\mathcal{S})$, there exists $s \in \mathcal{S}$ such that $\sigma=[s]$. Given a triangulated functor $F: \mathcal{S} \rightarrow \mathcal{S}^{\prime}$ between essentially small triangulated categories, there is an induced group homomorphism $\mathrm{K}_{0}(F): \mathrm{K}_{0}(\mathcal{S}) \rightarrow \mathrm{K}_{0}\left(\mathcal{S}^{\prime}\right)$. The following is a nice result of $\mathrm{A}$. Neeman [Nee92b, Cor. 0.10] (also see [TT90, 5.2.2] and [Tho97, Lem. 2.2]).

Lemma 3.3. Let $\mathcal{S}$ be an essentially small triangulated category and let $\mathcal{R}$ be a dense subcategory. If $s \in \mathcal{S}$, then $s \in \mathcal{R}$ if and only if $s$ belongs to the image of $\mathrm{K}_{0}(\mathcal{R})$ in $\mathrm{K}_{0}(\mathcal{S})$. In particular, if $s \in \mathcal{S}$, then $s \oplus \Sigma s \in \mathcal{R}$.

A pair of triangulated functors $\mathcal{R} \rightarrow \mathcal{S} \stackrel{F}{\rightarrow} \mathcal{T}$ is left-exact (resp. almost exact, resp. exact) if $\mathcal{R}$ is a thick subcategory of $\mathcal{S}$ via the functor $\mathcal{R} \rightarrow \mathcal{S}$ and the functor $F: \mathcal{S} \rightarrow \mathcal{T}$ factors through the quotient $\bar{F}: \mathcal{S} / \mathcal{R} \rightarrow \mathcal{T}$ and this functor is fully faithful (resp. fully faithful and dense, resp. an equivalence). The following (well-known) Lemma will be useful.

Lemma 3.4. Let $F: \mathcal{S} \rightarrow \mathcal{T}$ be a triangulated functor. If $F$ has a right adjoint $G: \mathcal{T} \rightarrow \mathcal{S}$ such that the adjunction $\epsilon: F G \rightarrow \mathrm{Id}_{\mathcal{T}}$ is an isomorphism, then the sequence $\operatorname{ker} F \rightarrow \mathcal{S} \stackrel{F}{\rightarrow} \mathcal{T}$ is exact.

Proof. We will show that $F$ satisfies the universal property of the quotient. Let $P: \mathcal{S} \rightarrow \mathcal{P}$ be a triangulated functor such that $\operatorname{ker} F \subseteq \operatorname{ker} P$. We must prove that there is a functor $P^{\prime}: \mathcal{T} \rightarrow \mathcal{P}$ and an isomorphism $\alpha: P \simeq P^{\prime} F$ unique up to unique isomorphism. For the uniqueness, let $P_{1}^{\prime}, P_{2}^{\prime}$ be two such functors with isomorphisms $\alpha_{i}: P \simeq P_{i}^{\prime} F$. Then the isomorphism $\alpha_{2} \alpha_{1}^{-1}: P_{1}^{\prime} F \simeq P_{2}^{\prime} F$ induces a unique isomorphism $P_{1}^{\prime} \simeq P_{1}^{\prime} F G \simeq P_{2}^{\prime} F G \simeq P_{2}^{\prime}$ compatible with the $\alpha_{i}$. For the existence, set $P^{\prime}=P G$ and let $\alpha=P_{*} \eta$, where $\eta$ : $\operatorname{Id}_{\mathcal{S}} \rightarrow G F$ is the unit of the adjunction. Now $F_{*} \eta$ is an isomorphism since $\epsilon$ is an isomorphism, so $\alpha=P_{*} \eta$ is an isomorphism since ker $F \subseteq \operatorname{ker} P$.

A triangulated category is said to be closed under small coproducts if it admits small categorical coproducts and small coproducts of triangles remain triangles. If the triangulated category $\mathcal{S}$ is closed under small coproducts, then we say that a subcategory $\mathcal{R} \subseteq \mathcal{S}$ is localizing if it is a full triangulated subcategory, closed under small coproducts, and the functor $\mathcal{R} \rightarrow \mathcal{S}$ preserves small coproducts. For a class $R \subseteq \mathcal{S}$, where $\mathcal{S}$ is closed under small coproducts, there is a smallest subcategory $\mathcal{R} \subseteq \mathcal{S}$ that is localizing and contains $R$. We refer to $\mathcal{R}$ as the localizing envelope of $R$. 
Example 3.5. If a subcategory $\mathcal{R} \subseteq \mathcal{S}$ is localizing, then it is thick. Indeed, given $r \in \mathcal{R}$ and $r \simeq r^{\prime} \oplus r^{\prime \prime}$ in $\mathcal{S}$, the Eilenberg swindle produces an $\mathcal{S}$-isomorphism:

$$
r^{\prime \prime} \oplus r \oplus r \oplus \cdots \simeq r \oplus r \oplus r \oplus \cdots .
$$

Since $\mathcal{R}$ is localizing, $r^{\prime \prime} \oplus r \oplus r \oplus \cdots \in \mathcal{R}$. The cone of the natural morphism $r \oplus r \oplus \cdots \rightarrow r^{\prime \prime} \oplus r \oplus r \oplus \cdots$ is $r^{\prime \prime}$; thus $r^{\prime \prime} \in \mathcal{R}$.

A result of A. Neeman [Nee92b, Prop. 1.9] says that if a subcategory $\mathcal{R} \subseteq \mathcal{S}$ is localizing, then the quotient $Q: \mathcal{S} \rightarrow \mathcal{S} / \mathcal{R}$ preserves small coproducts. In particular, since the quotient is essentially surjective, the category $\mathcal{S} / \mathcal{R}$ is closed under small coproducts.

Example 3.6. If $F: \mathcal{S} \rightarrow \mathcal{S}^{\prime}$ is a triangulated functor that preserves small coproducts, then the subcategory $\operatorname{ker} F$ is localizing.

An object $s \in \mathcal{S}$ is compact if the functor $\operatorname{Hom}_{\mathcal{S}}(s,-)$ preserves small coproducts. Denote by $\mathcal{S}^{c}$ the full subcategory of compact objects of $\mathcal{S}$.

Example 3.7. Let $A$ be a ring. A complex of $A$-modules is compact in $\mathrm{D}(A)$ if and only if it is quasi-isomorphic to a bounded complex of finitely generated projective $A$-modules [Stacks, 07LT]. That is, the compact objects of $\mathrm{D}(A)$ are the perfect complexes of $A$-modules.

Example 3.8. Let $F: \mathcal{S} \rightarrow \mathcal{S}^{\prime}$ be a triangulated functor that admits a right adjoint $G: \mathcal{S}^{\prime} \rightarrow \mathcal{S}$. If $G$ preserves small coproducts, then $F$ sends $\mathcal{S}^{c}$ to $\mathcal{S}^{\prime c}$ [Nee96, Thm. 5.1 " $\Rightarrow$ "].

Example 3.9. If $f: X^{\prime} \rightarrow X$ is a concentrated morphism of algebraic stacks, then $\mathrm{L} f_{\mathrm{qc}}^{*}$ sends $\mathrm{D}_{\mathrm{qc}}(X)^{c}$ to $\mathrm{D}_{\mathrm{qc}}\left(X^{\prime}\right)^{c}$. This follows by combining Example 3.8 with Theorem 2.6 3].

A class $S \subseteq \mathcal{S}$ is generating if given $x \in \mathcal{S}$ such that $\operatorname{Hom}_{\mathcal{S}}\left(\Sigma^{n} s, x\right)=0$ for all $s \in S$ and $n \in \mathbb{Z}$, then $x \simeq 0$. The triangulated category $\mathcal{S}$ is compactly generated if it admits a set of generators consisting of compact objects.

Example 3.10. Let $A$ be a ring. Denote the unbounded derived category of $A$ modules by $\mathrm{D}(A)$. Then the set $\{A\}$ compactly generates $\mathrm{D}(A)$. Hence $\mathrm{D}(A)$ is compactly generated.

Example 3.11. This is a refinement of Example 3.8. Let $F: \mathcal{S} \rightarrow \mathcal{S}^{\prime}$ be a triangulated functor that admits a right adjoint $G: \mathcal{S}^{\prime} \rightarrow \mathcal{S}$ that preserves small coproducts. In addition, assume that $G$ is conservative (i.e., $G(x) \simeq 0$ implies $x \simeq 0$ ). If $\mathcal{S}$ is compactly generated by a class $S$, then $\mathcal{S}^{\prime}$ is compactly generated by the class $\{F(s): s \in S\}$.

We now recall Thomason's Localization Theorem, which was proved in this generality by A. Neeman [Nee92b, Nee96, Thm. 2.1] (also see [TT90, 5.1]).

Theorem 3.12 (Thomason's Localization). Consider an exact sequence of triangulated categories $\mathcal{R} \rightarrow \mathcal{S} \stackrel{F}{\rightarrow} \mathcal{T}$ that are closed under small coproducts. If the triangulated category $\mathcal{S}$ is compactly generated and $\mathcal{R}$ is the localizing envelope of a subset $R \subseteq \mathcal{S}^{c}$, then there is an induced sequence $\mathcal{R}^{c} \rightarrow \mathcal{S}^{c} \rightarrow \mathcal{T}^{c}$ which is almost exact. In particular, $\mathcal{R}^{c}=\mathcal{S}^{c} \cap \mathcal{R}$ and $\mathcal{R}^{c}$ is the thick closure of $R$.

Combining Theorem 3.12 with the elementary Lemma 3.3 produces something very surprising, which was observed by A. Neeman [Nee92b, Cor. 0.9].

Corollary 3.13. In Theorem 3.12 assume that the category $\mathcal{T}^{c}$ is essentially small. Then for every $t \in \mathcal{T}^{c}$, there exists an $s \in \mathcal{S}^{c}$ and an isomorphism $t \oplus \Sigma t \simeq F(s)$. 
Another useful Corollary is the following [Nee96, Thm. 2.1.2].

Corollary 3.14. In Theorem 3.12 suppose that $R$ is a generating set for $\mathcal{S}$, then $\mathcal{R}=\mathcal{S}$.

\section{Perfect complexes, Projection formulas, and finite Duality}

We now use the results of the previous section to prove some useful results for derived categories of algebraic stacks. We begin with the notion of a perfect complex on an algebraic stack.

4.1. Perfect complexes. We recall some notions from SGA6, Exp. II] (also see Stacks, Tag $08 \mathrm{FK})$. If $A$ is a ring, then a complex of $A$-modules $P$ is strictly perfect if it is a bounded complex of finitely generated and projective $A$-modules. More generally, if $A$ is a sheaf of rings on a site $E$, then a complex $P=\left(P^{k}\right)$ of $A$-modules is strictly perfect if it is a bounded complex and each term $P^{k}$ is a direct summand of a finite free $A$-module. A complex $P \in \mathrm{D}(A)$ is perfect if it is locally strictly perfect.

Let $X$ be an algebraic stack. Then a complex $\mathcal{P}$ on $X$ is perfect if it is a perfect object of $\mathrm{D}(X)$. Note that all perfect complexes on $X$ belong to $\mathrm{D}_{\mathrm{qc}}(X)$. The following lemma provides a useful criterion for perfection.

Lemma 4.1. Let $X$ be an algebraic stack and let $\mathcal{P} \in \mathrm{D}_{\mathrm{qc}}(X)$. The following conditions are equivalent.

(1) $\mathcal{P}$ is perfect; and

(2) for every $x \in|X|$, there exists a flat morphism $f: \operatorname{Spec} A \rightarrow X$ with image containing $x$ such that $\mathrm{R} \Gamma\left(\operatorname{Spec} A, \mathrm{~L} f_{\mathrm{qc}}^{*} \mathcal{P}\right)$ is a strictly perfect complex of A-modules.

Moreover, every perfect complex on an affine scheme is strictly perfect.

Proof. It is sufficient to prove that if $A \rightarrow B$ is a faithfully flat ring homomorphism and $M \in \mathrm{D}(A)$, then $M$ is a strictly perfect complex of $A$-modules if and only if $M \otimes_{A} B$ is a strictly perfect complex of $B$-modules. This is Stacks, Tag 068T.

Example 4.2. Let $X$ be an algebraic stack. Then every flat $\mathcal{O}_{X}$-module of finite presentation defines a perfect complex in $\mathrm{D}_{\mathrm{qc}}(X)$. In particular, if $f: X \rightarrow Y$ is a morphism of algebraic stacks that is finite, flat, and of finite presentation, then $f_{*} \mathcal{O}_{X}$ is perfect in $\mathrm{D}_{\mathrm{qc}}(Y)$.

We recall the following well-known definition (e.g., Bra14, Defn. 4.7.1] or BZFN10, Defn. 3.3]). Let $X$ be an algebraic stack. An object $\mathcal{P} \in \mathrm{D}_{\mathrm{qc}}(X)$ is dualizable if there exists $\mathcal{P}^{\vee} \in \mathrm{D}_{\mathrm{qc}}(X)$ together with morphisms $e: \mathcal{P}^{\vee} \otimes_{\mathcal{O}_{X}}^{\mathrm{L}} \mathcal{P} \rightarrow \mathcal{O}_{X}$, $c: \mathcal{O}_{X} \rightarrow \mathcal{P} \otimes_{\mathcal{O}_{X}}^{\mathrm{L}} \mathcal{P}^{\vee}$ such that the two induced maps:

$$
\begin{aligned}
& \mathcal{O}_{X} \otimes_{\mathcal{O}_{X}}^{\mathrm{L}} \mathcal{P} \stackrel{c \otimes \mathrm{Id}_{\mathcal{P}}}{\longrightarrow} \mathcal{P} \otimes_{\mathcal{O}_{X}}^{\mathrm{L}} \mathcal{P}^{\vee} \otimes_{\mathcal{O}_{X}}^{\mathrm{L}} \mathcal{P} \stackrel{\mathrm{Id}_{\mathcal{P}} \otimes e}{\longrightarrow} \mathcal{P} \\
& \mathcal{P} \vee \otimes_{\mathcal{O}_{X}}^{\mathrm{L}} \mathcal{O}_{X} \stackrel{\operatorname{Id}_{\mathcal{P}} \vee \otimes C}{\longrightarrow} \mathcal{P}^{\vee} \otimes_{\mathcal{O}_{X}}^{\mathrm{L}} \mathcal{P} \otimes_{\mathcal{O}_{X}}^{\mathrm{L}} \mathcal{P} \stackrel{e}{ } \stackrel{e \otimes \mathrm{Id}_{\mathcal{P}} \vee}{\longrightarrow} \mathcal{P} \vee
\end{aligned}
$$

are isomorphisms. It is standard that $\mathcal{P}$ dualizable implies that $\mathcal{P}^{\vee} \simeq \mathrm{R} \mathcal{H} \operatorname{com}_{\mathcal{O}_{X}}^{\mathrm{qc}}\left(\mathcal{P}, \mathcal{O}_{X}\right)$ and the $e$ and $c$ maps are simply those arising from the adjunction between $-\otimes_{\mathcal{O}_{X}}^{\mathrm{L}} \mathcal{P}$ and $\mathrm{R} \mathcal{H} \operatorname{Tom}_{\mathcal{O}_{X}}^{\mathrm{qc}}(\mathcal{P},-)$.

The following Lemma is straightforward but crucial.

Lemma 4.3. Let $X$ be an algebraic stack and let $\mathcal{P} \in \mathrm{D}_{\mathrm{qc}}(X)$ be a perfect complex.

(1) The double duality morphism $\mathcal{P} \rightarrow \mathrm{RH} \operatorname{Com}_{\mathcal{O}_{X}}\left(\mathrm{RHom}_{\mathcal{O}_{X}}\left(\mathcal{P}, \mathcal{O}_{X}\right), \mathcal{O}_{X}\right)$ is a quasi-isomorphism. 
(2) The restriction of the functor $\mathrm{RH} \mathcal{H}_{\mathcal{O}_{X}}(\mathcal{P},-): \mathrm{D}(X) \rightarrow \mathrm{D}(X)$ to $\mathrm{D}_{\mathrm{qc}}(X)$ factors through $\mathrm{D}_{\mathrm{qc}}(X)$ and preserves small coproducts in $\mathrm{D}_{\mathrm{qc}}(X)$. Moreover, if $\mathcal{M} \in \mathrm{D}_{\mathrm{qc}}(X)$, then there is a natural quasi-isomorphism:

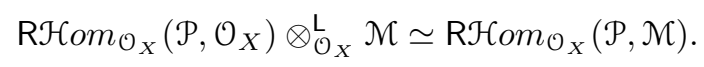

In particular, $\mathrm{R} \mathcal{H} \mathcal{H o m}_{\mathcal{O}_{X}}^{\mathrm{qc}}(\mathcal{P},-) \simeq \mathrm{RH}^{-} m_{\mathcal{O}_{X}}(\mathcal{P},-) \simeq \mathcal{P}^{\vee} \otimes-$ on $\mathrm{D}_{\mathrm{qc}}(X)$.

(3) If $\mathcal{P} \in \mathrm{D}_{\mathrm{qc}}(X)$ is dualizable, then it is perfect.

In particular for every algebraic stack $X$, the notions of perfect and dualizable objects in $\mathrm{D}_{\mathrm{qc}}(X)$ coincide.

Proof. To prove (1), we note that the existence of the double duality morphism follows from $\mathrm{RH}(\mathrm{H}-\otimes$ adjunction. That it is a quasi-isomorphism can be verified smooth-locally on $X$, so we may assume that $X=\operatorname{Spec} A$ is an affine scheme. To prove (2), we may argue similarly to reduce to the affine setting. Now the collection of all $\mathcal{P}$ that satisfy the conclusions of (1) and $(2)$ is closed under finite coproducts, direct summands, shifts, and the taking of cones, that is, it is a thick subcategory of $\mathrm{D}_{\mathrm{qc}}(X) \simeq \mathrm{D}(A)$. Since $\mathcal{O}_{X}$ satisfies the conclusions of (1) and (2) and $X$ is affine, (1) and (2) follow.

For (3): since dualizable implies locally dualizable, it follows that we may assume that $X$ is affine. In this case, the result is classical. One may also argue as follows: $Q$ being dualizable implies that there is an isomorphism of functors

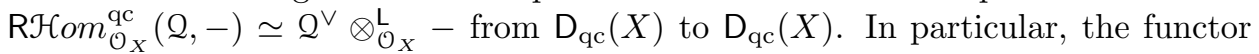
$\mathrm{RH} \mathcal{H o m}_{\mathcal{O}_{X}}^{\mathrm{qc}}(Q,-): \mathrm{D}_{\mathrm{qc}}(X) \rightarrow \mathrm{D}_{\mathrm{qc}}(X)$ preserves small coproducts. Taking global sections, we see that $\operatorname{Hom}_{\mathcal{O}_{X}}(Q,-)$ preserves small coproducts. Hence, $Q$ is compact in $\mathrm{D}_{\mathrm{qc}}(X)$. But $X$ is affine, so $Q$ is perfect.

4.2. Compact complexes. We now move on to a description of the compact objects of $\mathrm{D}_{\mathrm{qc}}(X)$ and their relationship to perfect complexes.

Lemma 4.4. Let $X$ be a quasi-separated algebraic stack.

(1) If $\mathcal{Q} \in \mathrm{D}_{\mathrm{qc}}(X)^{c}$, then $\mathrm{Q}$ is perfect.

(2) If $\mathcal{P}$ is perfect on $X$ and $\mathcal{Q} \in \mathrm{D}_{\mathrm{qc}}(X)^{c}$, then $\mathcal{Q} \otimes_{\mathcal{O}_{X}}^{\mathrm{L}} \mathcal{P} \in \mathrm{D}_{\mathrm{qc}}(X)^{c}$.

(3) If $X$ is concentrated, then $\mathcal{O}_{X} \in \mathrm{D}_{\mathrm{qc}}(X)^{c}$; in particular, if $\mathcal{P}$ is perfect on $X$, then $\mathcal{P} \in \mathrm{D}_{\mathrm{qc}}(X)^{c}$.

Proof. We first prove (1). Consider a smooth morphism $p$ : Spec $A \rightarrow X$. It follows from the quasi-separatedness of $X$ that $p$ is quasi-compact, quasi-separated, and representable. In particular, $p$ is concentrated (Lemma 2.5 (3) ) and so $L p_{\mathrm{qc}}^{*} \mathcal{Q} \in$ $\mathrm{D}_{\mathrm{qc}}(\operatorname{Spec} A)^{c}$ by Example 3.9. The claim now follows from Example 3.7 and Lemma 4.1 .

To prove (2), we note that if $\mathcal{M} \in \mathrm{D}(X)$, then 1.3 implies

$$
\operatorname{Hom}_{\mathcal{O}_{X}}\left(\mathcal{Q} \otimes_{\mathcal{O}_{X}}^{\mathrm{L}} \mathcal{P}, \mathcal{M}\right) \simeq \operatorname{Hom}_{\mathcal{O}_{X}}\left(\mathcal{Q}, \mathrm{R} \mathcal{H} \operatorname{Hom}_{\mathcal{O}_{X}}(\mathcal{P}, \mathcal{M})\right) .
$$

By Lemma 4.322, the restriction of $\mathrm{R} \mathcal{H} \operatorname{Com}(\mathcal{P},-)$ to $\mathrm{D}_{\mathrm{qc}}(X)$ preserves small coproducts. Since $\mathcal{Q} \in \mathrm{D}_{\mathrm{qc}}(X)^{c}$, it follows that the restriction of $\mathrm{RH}_{\mathcal{H}} \operatorname{com}_{\mathcal{O}_{X}}\left(\mathcal{Q} \otimes_{\mathcal{O}_{X}}^{\mathrm{L}} \mathcal{P},-\right)$ to $\mathrm{D}_{\mathrm{qc}}(X)$ preserves small coproducts. Hence, $\mathcal{Q} \otimes_{\mathcal{O}_{X}}^{\mathrm{L}} \mathcal{P} \in \mathrm{D}_{\mathrm{qc}}(X)^{c}$.

For (3), we note that if $\mathcal{M} \in \mathrm{D}(X)$, then by definition $\operatorname{RHom}_{\mathcal{O}_{X}}\left(\mathcal{O}_{X}, \mathcal{M}\right)=$ $\mathrm{R} \Gamma\left(X_{\text {lis-ét }}, \mathcal{M}\right)$. Since $X$ is concentrated, Theorem 2.6 3) implies that the restriction of $\mathrm{R} \Gamma\left(X_{\text {lis-ét }},-\right)$ to $\mathrm{D}_{\mathrm{qc}}(X)$ preserves small coproducts. Thus, $\mathcal{O}_{X} \in \mathrm{D}_{\mathrm{qc}}(X)^{c}$. The latter claim follows from the former and (2).

In the following Lemma we provide criteria for a perfect complex on an algebraic stack to be compact. We have not seen this characterization in the literature before. 
Lemma 4.5. Let $X$ be a quasi-compact and quasi-separated algebraic stack and let $\mathcal{P} \in \mathrm{D}_{\mathrm{qc}}(X)$ be a perfect complex. The following conditions are equivalent:

(1) $\mathcal{P}$ is a compact object of $\mathrm{D}_{\mathrm{qc}}(X)$;

(2) there exists an integer $r \geq 0$ such that $\operatorname{Hom}_{\mathcal{O}_{X}}(\mathcal{P}, \mathcal{N}[i])=0$ for all $\mathcal{N} \in$ $\mathrm{QCoh}(X)$ and $i>r$; and

(3) there exists an integer $r \geq 0$ such that the natural map

$$
\tau^{\geq j} \operatorname{RHom}_{\mathcal{O}_{X}}(\mathcal{P}, \mathcal{M}) \rightarrow \tau^{\geq j} \operatorname{RHom}_{\mathcal{O}_{X}}\left(\mathcal{P}, \tau^{\geq j-r} \mathcal{M}\right)
$$

is a quasi-isomorphism for all $\mathcal{M} \in \mathrm{D}_{\mathrm{qc}}(X)$ and integers $j$.

Proof. Assume that (2) does not hold. Then there is an infinite sequence of quasicoherent $\mathcal{O}_{X}$-modules $\mathcal{M}_{1}, \mathcal{M}_{2}, \ldots$ and strictly increasing sequence of integers $d_{1}<$ $d_{2}<\ldots$ such that $\operatorname{Hom}_{\mathcal{O}_{X}}\left(\mathcal{P}, \mathcal{M}_{i}\left[d_{i}\right]\right) \neq 0$ for every $i$. Since $\mathrm{D}_{\mathrm{qc}}(X)$ is left-complete HNR14, Thm. B.1], there is a quasi-isomorphism in $\mathrm{D}_{\mathrm{qc}}(X)$ :

$$
\bigoplus_{i=1}^{\infty} \mathcal{M}_{i}\left[d_{i}\right] \simeq \prod_{i=1}^{\infty} \mathcal{M}_{i}\left[d_{i}\right]
$$

This implies that the natural morphism

$$
\bigoplus_{i=1}^{\infty} \operatorname{Hom}_{\mathcal{O}_{X}}\left(\mathcal{P}, \mathcal{M}_{i}\left[d_{i}\right]\right) \rightarrow \operatorname{Hom}_{\mathcal{O}_{X}}\left(\mathcal{P}, \bigoplus_{i=1}^{\infty} \mathcal{M}_{i}\left[d_{i}\right]\right) \simeq \prod_{i=1}^{\infty} \operatorname{Hom}_{\mathcal{O}_{X}}\left(\mathcal{P}, \mathcal{M}_{i}\left[d_{i}\right]\right)
$$

is not an isomorphism. In particular, $\mathcal{P}$ is not compact. Thus, by the contrapositive, we have proved the implication $(1) \Rightarrow(2)$.

For $(2) \Rightarrow(3)$, first choose a diagram as in 1.6 with $V=Y=\operatorname{Spec} \mathbb{Z}$. Let $\mathcal{P}_{\bullet, \text { ét }}^{+}=\overline{\mathrm{R}}\left(\operatorname{res}_{U_{\bullet}}\right)_{*} \mathrm{~L}\left(p_{\bullet, \text { lis-ét }}^{+}\right) * \mathcal{P}$ and $\mathcal{M}_{\bullet, \text { ét }}^{+}=\mathrm{R}\left(\operatorname{res}_{U_{\bullet}}\right)_{*} \mathrm{~L}\left(p_{\bullet, \text { lis-ét }}^{+}\right) * \mathcal{M}$. Note that

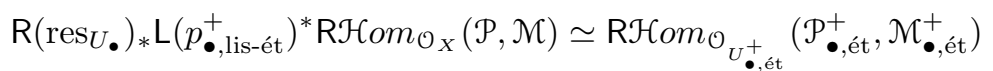

has quasi-coherent cohomology (Lemma 4.3 and that $\operatorname{RHom}_{\mathcal{O}_{U_{\bullet}^{+}}^{+}}\left(\mathcal{P}_{\bullet}^{+}\right.$,ét, $\left.\mathcal{N}[i]\right)=0$ for all $i>r$ and $\mathcal{N} \in \mathrm{Q} \operatorname{Coh}\left(U_{\bullet \text {,ét }}^{+}\right)$. It is enough to prove that

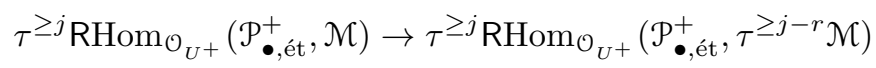

for every integer $j$ and $\mathcal{M} \in \mathrm{D}_{\mathrm{qc}}\left(U_{\bullet}^{+}\right.$,ét $)$. This follows as in the proof of [LO08, Lem. 2.1.10] with $\epsilon_{*}$ replaced by $\operatorname{Hom}_{\mathcal{O}_{U_{\bullet}^{+}}^{+}}\left(\mathcal{P}_{\bullet, \text { ét }}^{+},-\right)$.

Finally, for $(3) \Rightarrow(1)$, we note that because $\mathcal{P}$ is perfect, it is dualizable. Thus, for all $\mathcal{M} \in \mathrm{D}_{\mathrm{qc}}(X)$ and integers $j$, there are natural quasi-isomorphisms:

$$
\begin{aligned}
\tau^{\geq j} \operatorname{RHom}_{\mathcal{O}_{X}}(\mathcal{P}, \mathcal{M}) & \simeq \tau^{\geq j} \operatorname{RHom}_{\mathcal{O}_{X}}\left(\mathcal{P}, \tau^{\geq j-r} \mathcal{M}\right) \\
& \simeq \tau^{\geq j} \operatorname{R} \Gamma\left(X_{\text {lis-ét }}, \mathcal{P}^{\vee} \otimes_{\mathcal{O}_{X}}^{\mathrm{L}} \tau^{\geq j-r} \mathcal{M}\right) .
\end{aligned}
$$

Also since $\mathcal{P}$ is perfect, the restriction of the functor $\mathcal{P}^{\vee} \otimes_{\mathcal{O}_{X}}^{\mathrm{L}}(-)$ to $\mathrm{D}_{\mathrm{qc}}^{\geq j-r}(X)$ factors through $\mathrm{D}_{\mathrm{qc}}^{\geq j-r^{\prime}}(X)$ for some fixed integer $r^{\prime}$. The result now follows from Lemma 1.2.3.

For future reference, we summarize the situation in the following remark.

Remark 4.6. Let $X$ be a quasi-compact and quasi-separated algebraic stack. Then the following are equivalent:

(1) every perfect object of $\mathrm{D}_{\mathrm{qc}}(X)$ is compact;

(2) the structure sheaf $\mathcal{O}_{X}$ is compact;

(3) $X$ has finite cohomological dimension; and

(4) the derived global section functor $\mathrm{R} \Gamma: \mathrm{D}_{\mathrm{qc}}(X) \rightarrow \mathrm{D}(\mathrm{Ab})$ commutes with small coproducts. 
The equivalence of the first two conditions follows from Lemma 4.4. The structure sheaf is compact, if and only if $X$ has finite cohomological dimension (Lemma 4.5). The last condition is equivalent to the definition of $\mathcal{O}_{X}$ being compact.

4.3. Supports and generation. Let $X$ be a scheme and let $M \in \mathrm{Q} \operatorname{Coh}(X)$. The support of $M$ is the subset

$$
\operatorname{supp}(M)=\left\{x \in|X|: M_{x} \neq 0\right\} .
$$

More generally, if $X$ is an algebraic stack and $M \in \mathrm{Q} \operatorname{Coh}(X)$, then we define the support of $M$ as follows: let $p: U \rightarrow X$ be a smooth surjection from a scheme $U$; then $\operatorname{supp}(M)=p\left(\operatorname{supp}\left(p^{*} M\right)\right)$. It is easily verified that this is well-defined (i.e., independent of the cover $p)$.

Definition 4.7. Let $X$ be an algebraic stack and let $E \in \mathrm{D}_{\mathrm{qc}}(X)$. Define the cohomological support of $E$ to be the subset

$$
\operatorname{supph}(E)=\cup_{n \in \mathbb{Z}} \operatorname{supp}\left(\mathcal{H}^{n}(E)\right) \subseteq|X| .
$$

Recall that if $X$ is a quasi-separated algebraic stack, then for every $x \in|X|$, there is a quasi-affine monomorphism $i_{x}: \mathcal{G}_{x} \hookrightarrow X$ with image $x$, where $\mathcal{G}_{x}$ is a gerbe over a field $\kappa(x)$, the residue field at $x$ [Ryd11, Thm. B.2]. We refer to this data as the residual gerbe at $x$. We now have the following lemma.

Lemma 4.8. Let $X$ be a quasi-compact and quasi-separated algebraic stack. Let $E$ be a perfect complex on $X$.

(1) Then $x \in \operatorname{supph}(E)$ if and only if the complex $\mathrm{L}\left(i_{x}\right)_{\mathrm{qc}}^{*} E$ is not acyclic in $\mathrm{D}_{\mathrm{qc}}\left(\mathcal{G}_{x}\right)$, where $i_{x}: \mathcal{G}_{x} \rightarrow X$ is the residual gerbe.

(2) If $f: X^{\prime} \rightarrow X$ is a quasi-compact and quasi-separated morphism, then $\operatorname{supph}\left(\mathrm{L} f_{\mathrm{qc}}^{*} E\right)=f^{-1} \operatorname{supph}(E)$.

(3) $\operatorname{supph}(E)$ is closed with quasi-compact complement.

Proof. For (1), then $x \in \operatorname{supph}(E)$, if and only if there exists a smooth and surjective morphism $p: U \rightarrow X$, where $U$ is a scheme, and $u \in U$ such that $p(u)=x$ and $\left(\mathrm{L} p_{\mathrm{qc}}^{*} E\right)_{u}$ is not acyclic in $\mathrm{D}_{\mathrm{qc}}\left(\mathcal{O}_{U, u}\right)$. By [Tho97, Lem. 3.3(a)], this is equivalent to $\left(\mathrm{L} p_{\mathrm{qc}}^{*} E\right) \otimes \kappa(u)$ not being acyclic in $\mathrm{D}_{\mathrm{qc}}(\kappa(u))$. Since $\operatorname{Spec} \kappa(u) \rightarrow \mathcal{G}_{x}$ is faithfully flat, the result follows.

For (2), we use (1) and argue as in [Tho97, Lem. 3.3(b)], with the additional observation that for every $x^{\prime} \in\left|X^{\prime}\right|$, the induced morphism on residual gerbes $\mathcal{G}_{x^{\prime}} \rightarrow \mathcal{G}_{f\left(x^{\prime}\right)}$ is faithfully flat.

For (3), by (2), the conclusion may be verified smooth-locally. In particular, we may assume that $X$ is an affine scheme, and the result follows from Tho97, Lem. 3.3(c)].

If $X$ is an algebraic stack and $Z \subseteq|X|$, then we define

$$
\mathrm{D}_{\mathrm{qc},|Z|}(X)=\left\{\mathcal{M} \in \mathrm{D}_{\mathrm{qc}}(X): \operatorname{supph}(\mathcal{M}) \subseteq Z\right\}
$$

If $j: U \hookrightarrow X$ is a flat monomorphism, e.g., an open immersion, then

$$
\mathrm{D}_{\mathrm{qc},|X \backslash U|}(X)=\left\{\mathcal{M} \in \mathrm{D}_{\mathrm{qc}}(X): j^{*} \mathcal{M} \simeq 0\right\} .
$$

Lemma 4.9. Let $X$ be an algebraic stack and let $\mathcal{P} \in \mathrm{D}_{\mathrm{qc}}(X)$ be a perfect complex with support $Z=\operatorname{supph}(\mathcal{P})$. If $\mathcal{M} \in \mathrm{D}_{\mathrm{qc},|Z|}(X)$, then $\mathcal{M} \simeq 0$ if and only if $\mathrm{RH} \operatorname{com}_{\mathcal{O}_{X}}(\mathcal{P}, \mathcal{M}) \simeq 0$.

Proof. The question is local on $X$ for the smooth topology, so we may assume that $X$ is an affine scheme. By BN93, Prop. 6.1], there is a perfect generating complex $K \in \mathrm{D}_{\mathrm{qc},|Z|}(X)^{c}$ (a Koszul complex). As $\mathcal{P}$ is perfect and the support of $\mathcal{P}$ is $|Z|$, it follows from [Nee92a, Lem. A.3] that $K$ is in the thick closure of $\mathcal{P}$, so $\mathcal{P}$ is also a generator of $\mathrm{D}_{\mathrm{qc},|Z|}(X)$. Thus, $\operatorname{RHom}_{\mathcal{O}_{X}}(\mathcal{P}, \mathcal{M}) \simeq 0$ if and only if $\mathcal{M} \simeq 0$. 
Lemma 4.10. Let $X$ be a quasi-compact and quasi-separated algebraic stack and let $j: U \rightarrow X$ be a quasi-compact open immersion with complement $|Z|$.

(1) If $\mathrm{D}_{\mathrm{qc},|Z|}(X)$ is generated by a set whose elements have compact image in $\mathrm{D}_{\mathrm{qc}}(X)$, then there exists a compact object $\mathcal{Q}$ of $\mathrm{D}_{\mathrm{qc}}(X)$ with support $|Z|$.

(2) If $\mathrm{D}_{\mathrm{qc}}(X)$ is generated by a set of compact objects $\left\{Q_{b}\right\}_{b \in B}$ and there exists a perfect complex $\mathcal{P}$ on $X$ with support $|Z|$, then $\mathrm{D}_{\mathrm{qc},|Z|}(X)$ is generated by the set $\left\{\mathcal{Q}_{b} \otimes_{\mathcal{O}_{X}}^{\mathrm{L}} \mathcal{P}\right\}_{b \in B}$ (whose elements have compact image in $\mathrm{D}_{\mathrm{qc}}(X)$ ).

Proof. For (1): let $\left\{\mathcal{Q}_{\lambda}\right\}_{\lambda \in \Lambda}$ be a set of generators for $\mathrm{D}_{\mathrm{qc},|Z|}(X)$ whose elements have compact image in $\mathrm{D}_{\mathrm{qc}}(X)$. Let $z \in|Z|$ be a point and choose a representative, that is, a field $k$ and a 1-morphism of algebraic stacks $\bar{z}$ : Spec $k \rightarrow X$ with image $z$. Since the diagonal of $X$ is quasi-compact and quasi-separated, it follows that $\bar{z}$ is quasi-compact and quasi-separated. By Lemma 1.2(4), it follows that $\mathrm{L} j_{\mathrm{qc}}^{*} \mathrm{R}\left(\bar{z}_{\mathrm{qc}}\right)_{*} \mathcal{O}_{\text {Spec } k} \simeq 0$ and so there exists a $\lambda \in \Lambda$, an integer $n$, and a non-zero morphism $\mathcal{Q}_{\lambda}[n] \rightarrow \mathrm{R}\left(\bar{z}_{\mathrm{qc}}\right)_{*} \mathcal{O}_{\text {Spec } k}$. By adjunction, $\mathrm{L} \bar{z}_{\mathrm{qc}}^{*} \mathcal{Q}_{\lambda} \neq 0$ and we deduce that $\cup_{\lambda \in \Lambda} \operatorname{supph}\left(Q_{\lambda}\right)=|Z|$. It suffices to show that there is a finite subset $\Lambda^{\prime} \subseteq \Lambda$ such that $\cup_{\lambda \in \Lambda^{\prime}} \operatorname{supph}\left(Q_{\lambda}\right)=|Z|$. This is obvious if $X$ is noetherian or, more generally, if $Z$ has a finite number of irreducible components.

In complete generality, we note that $|Z|$ is constructible (by hypothesis) and that $\operatorname{supph}\left(Q_{\lambda}\right)$ is constructible for every $\lambda$ (by Lemma 4.8 3p). Indeed, both subsets are closed with quasi-compact complement. We conclude that $|Z|=\cup_{\lambda \in \Lambda} \operatorname{supph}\left(Q_{\lambda}\right)$ has a finite subcovering since the constructible topology is quasi-compact.

For (2): first note that the complex $\mathcal{Q}_{b} \otimes_{\mathcal{O}_{X}}^{\mathrm{L}} \mathcal{P}$, which belongs to $\mathrm{D}_{\mathrm{qc},|Z|}(X)$, is a compact object of $\mathrm{D}_{\mathrm{qc}}(X)$ (Lemma 4.4 2). Let $\mathcal{M} \in \mathrm{D}_{\mathrm{qc},|Z|}(X)$ and suppose that $\operatorname{RHom}_{\mathcal{O}_{X}}\left(\mathcal{Q}_{b} \otimes_{\mathcal{O}_{X}}^{\mathrm{L}} \mathcal{P}, \mathcal{M}\right) \simeq 0$. By adjunction $(1.3), \operatorname{RHom}_{\mathcal{O}_{X}}\left(\mathcal{Q}_{b}, \operatorname{RH}_{\mathcal{H}^{\prime}} \operatorname{Om}_{\mathcal{O}_{X}}(\mathcal{P}, \mathcal{M})\right) \simeq$ 0 . Since the set $\left\{\mathcal{Q}_{b}\right\}_{b \in B}$ is generating, it follows that $\mathrm{R} \mathcal{H} \operatorname{Com}_{\mathcal{O}_{X}}(\mathcal{P}, \mathcal{M}) \simeq 0$. Thus $\mathcal{M} \simeq 0$ by Lemma 4.9 .

4.4. Projection formula. A typical application of Corollary 3.14 is given by the following Proposition. The given argument is a variant of [Nee96, Prop. 5.3], though we have not seen this Proposition in the literature before.

Proposition 4.11 (Strong projection formula). Let $A$ be a ring and let $\pi: X \rightarrow$ Spec $A$ be a morphism of algebraic stacks. Let $\mathcal{Q}$ be a compact object of $\mathrm{D}_{\mathrm{qc}}(X)$ and let $\mathcal{G} \in \mathrm{D}_{\mathrm{qc}}(X)$. Then for every $I \in \mathrm{D}(A)$, there is a natural quasi-isomorphism:

$$
\operatorname{RHom}_{\mathcal{O}_{X}}(\mathcal{Q}, \mathcal{G}) \otimes_{A}^{\mathrm{L}} I \simeq \operatorname{RHom}_{\mathcal{O}_{X}}\left(\mathcal{Q}, \mathcal{G} \otimes_{\mathcal{O}_{X}}^{\mathrm{L}} \mathrm{L} \pi_{\mathrm{qc}}^{*} I\right)
$$

Proof. First we describe the morphism: by adjunction, there is a natural morphism

$$
\mathrm{L} \pi_{\mathrm{qc}}^{*} \mathrm{R}\left(\pi_{\mathrm{qc}}\right)_{*} \mathrm{RH} \operatorname{Hom}_{\mathcal{O}_{X}}(\mathcal{Q}, \mathcal{G}) \rightarrow \mathrm{RH} \operatorname{Hom}_{\mathcal{O}_{X}}(\mathcal{Q}, \mathcal{G})
$$

and so by 1.3 there is a natural morphism

$$
\left(\mathrm{L} \pi_{\mathrm{qc}}^{*} \mathrm{R}\left(\pi_{\mathrm{qc}}\right)_{*} \mathrm{RH}_{\mathcal{H}} \operatorname{mom}_{\mathcal{O}_{X}}(\mathcal{Q}, \mathcal{G})\right) \otimes_{\mathcal{O}_{X}}^{\mathrm{L}} \mathrm{Q} \otimes_{\mathcal{O}_{X}}^{\mathrm{L}} \mathrm{L} \pi_{\mathrm{qc}}^{*} I \rightarrow \mathcal{G} \otimes_{\mathcal{O}_{X}}^{\mathrm{L}} \mathrm{L} \pi_{\mathrm{qc}}^{*} I .
$$

By 1.3 again, there is a natural morphism:

$$
\mathrm{L} \pi_{\mathrm{qc}}^{*}\left[\mathrm{R}\left(\pi_{\mathrm{qc}}\right)_{*} \mathrm{R} \mathcal{H} \operatorname{Fom}_{\mathcal{O}_{X}}(\mathcal{Q}, \mathcal{G}) \otimes_{A}^{\mathrm{L}} I\right] \rightarrow \mathrm{R} \mathcal{H} \operatorname{com}_{\mathcal{O}_{X}}\left(\mathcal{Q}, \mathcal{G} \otimes_{\mathcal{O}_{X}}^{\mathrm{L}} \mathrm{L} \pi_{\mathrm{qc}}^{*} I\right) .
$$

By adjunction and 1.5 and 1.10 we deduce the existence of the required natural morphism

$$
\phi_{I}: \operatorname{RHom}_{\mathcal{O}_{X}}(2, \mathcal{G}) \otimes_{A}^{\mathrm{L}} I \rightarrow \operatorname{RHom}_{\mathcal{O}_{X}}\left(2, \mathcal{G} \otimes_{\mathcal{O}_{X}}^{\mathrm{L}} \mathrm{L} \pi_{\mathrm{qc}}^{*} I\right)
$$

Let $\mathcal{K} \subseteq \mathrm{D}(A)$ be the full subcategory with objects those $I$ such that $\phi_{I}$ is a quasiisomorphism. It remains to show that $\mathcal{K}=\mathrm{D}(A)$. Clearly, $\mathcal{K}$ is a triangulated subcategory that contains $A[k]$ for every integer $k$. Moreover, since $\mathcal{Q}$ is a compact object of $\mathrm{D}_{\mathrm{qc}}(X), \mathcal{K}$ is closed under small coproducts. The result now follows from Corollary 3.14 . 
A straightforward implication is the usual projection formula for concentrated morphisms of algebraic stacks.

Corollary 4.12 (Projection formula). Let $f: X \rightarrow Y$ be a concentrated 1-morphism of algebraic stacks. The natural map

$$
\left(\mathrm{R}\left(f_{\mathrm{qc}}\right)_{*} \mathcal{M}\right) \otimes_{\mathrm{O}_{Y}}^{\mathrm{L}} \mathcal{N} \rightarrow \mathrm{R}\left(f_{\mathrm{qc}}\right)_{*}\left(\mathcal{M} \otimes_{\mathrm{O}_{X}}^{\mathrm{L}} \mathrm{L} f_{\mathrm{qc}}^{*} \mathcal{N}\right)
$$

is a quasi-isomorphism for every $\mathcal{M} \in \mathrm{D}_{\mathrm{qc}}(X)$ and $\mathcal{N} \in \mathrm{D}_{\mathrm{qc}}(Y)$.

Proof. By adjunction, there is a natural morphism

$$
\mathrm{L} f_{\mathrm{qc}}^{*} \mathrm{R}\left(f_{\mathrm{qc}}\right)_{*} \mathcal{M} \otimes_{\mathcal{O}_{X}}^{\mathrm{L}} \mathrm{L} f_{\mathrm{qc}}^{*} \mathcal{N} \rightarrow \mathcal{M} \otimes_{\mathcal{O}_{X}}^{\mathrm{L}} \mathrm{L} f_{\mathrm{qc}}^{*} \mathcal{N} .
$$

By adjunction again, we deduce the existence of a natural morphism

$$
\psi_{\mathcal{N}}:\left(\mathrm{R}\left(f_{\mathrm{qc}}\right)_{*} \mathcal{M}\right) \otimes_{\mathcal{O}_{Y}}^{\mathrm{L}} \mathcal{N} \rightarrow \mathrm{R}\left(f_{\mathrm{qc}}\right)_{*}\left(\mathcal{M} \otimes_{\mathcal{O}_{X}}^{\mathrm{L}} \mathrm{L} f_{\mathrm{qc}}^{*} \mathcal{N}\right) .
$$

It remains to show that $\psi_{\mathcal{N}}$ is a quasi-isomorphism for every $\mathcal{N} \in \mathrm{D}_{\mathrm{qc}}(Y)$. Note that the verification of this is smooth local on $Y$, so by Theorem 2.6 (4), we may reduce to the situation where $Y$ is an affine scheme. By Lemma 4.4 (3), $\mathcal{O}_{X}$ is compact and the result now follows from Proposition 4.11 .

4.5. Tor-independent base change. Let $f: X \rightarrow Y$ and $g: Y^{\prime} \rightarrow Y$ be morphism of algebraic stacks. We say that $f$ and $g$ are tor-independent if for every smooth morphism Spec $A \rightarrow Y$ and every pair of smooth morphisms $\operatorname{Spec} B \rightarrow$ $X \times_{Y} \operatorname{Spec} A$ and $\operatorname{Spec} A^{\prime} \rightarrow Y^{\prime} \times_{Y} \operatorname{Spec} A$ we have that $\operatorname{Tor}_{i}^{A}\left(B, A^{\prime}\right)=0$ for all $i>0$. Equivalently, $\operatorname{Tor}_{i}^{Y, f, g}\left(\mathcal{O}_{X}, \mathcal{O}_{Y^{\prime}}\right)=0$ for every integer $i>0$ (see [Hal17, App. C] for details). Note that if $g$ is flat, then it is tor-independent of every $f$. The projection formula of Corollary 4.12 is powerful enough to prove a very general tor-independent base change result which extends Theorem 2.6 (4).

Corollary 4.13. Fix a 2-cartesian square of algebraic stacks

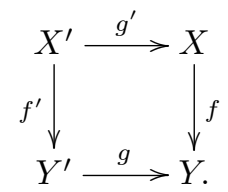

If $f$ and $g$ are tor-independent and $f$ is concentrated, then there is a natural quasiisomorphism for every $\mathcal{M} \in \mathrm{D}_{\mathrm{qc}}(X)$ :

$$
\mathrm{L} g_{\mathrm{qc}}^{*} \mathrm{R}\left(f_{\mathrm{qc}}\right)_{*} \mathcal{M} \simeq \mathrm{R}\left(f_{\mathrm{qc}}^{\prime}\right)_{*} \mathrm{~L} g_{\mathrm{qc}}^{\prime *} \mathcal{M}
$$

Proof. By Theorem 2.6 (4), the result can be verified smooth-locally on $Y$ and $Y^{\prime}$. Thus, we may assume that $Y=\operatorname{Spec} A$ and $Y^{\prime}=$ Spec $A^{\prime}$. In particular, $g$ and $g^{\prime}$ are affine. Since $g$ is affine, $\mathrm{R}\left(g_{\mathrm{qc}}\right)_{*}$ is conservative (Corollary 2.8). Hence, it is sufficient to verify that the morphism in question is a quasi-isomorphism after application of the functor $\mathrm{R}\left(g_{\mathrm{qc}}\right)_{*}$. By the projection formula applied to $g$ and then $f$, there are natural quasi-isomorphisms

$$
\begin{aligned}
\mathrm{R}\left(g_{\mathrm{qc}}\right)_{*} \mathrm{~L} g_{\mathrm{qc}}^{*} \mathrm{R}\left(f_{\mathrm{qc}}\right)_{*} \mathcal{M} & \simeq \mathrm{R}\left(f_{\mathrm{qc}}\right)_{*} \mathcal{M} \otimes_{\mathcal{O}_{Y}} \mathrm{R}\left(g_{\mathrm{qc}}\right)_{*} \mathcal{O}_{Y^{\prime}} \\
& \simeq \mathrm{R}\left(f_{\mathrm{qc}}\right)_{*}\left(\mathcal{M} \otimes_{\mathcal{O}_{X}}^{\mathrm{L}} \mathrm{L} f_{\mathrm{qc}}^{*} \mathrm{R}\left(g_{\mathrm{qc}}\right)_{*} \mathcal{O}_{Y^{\prime}}\right) .
\end{aligned}
$$

Note, however, that because $f$ and $g$ are tor-independent and $g$ is affine, the natural map

$$
\mathrm{L} f_{\mathrm{qc}}^{*} \mathrm{R}\left(g_{\mathrm{qc}}\right)_{*} \mathcal{O}_{Y^{\prime}} \rightarrow \mathrm{R}\left(g_{\mathrm{qc}}^{\prime}\right)_{*} \mathcal{O}_{X^{\prime}}
$$

is a quasi-isomorphism. Indeed, this may be verified smooth-locally on $X$, so we may assume that $X=\operatorname{Spec} C$. The morphism in question corresponds to the map $C \otimes_{A}^{\mathrm{L}} A^{\prime} \rightarrow\left(C \otimes_{A} A^{\prime}\right)[0]$ in $\mathrm{D}(C)$, which is a quasi-isomorphism because $f$ and $g$ 
are tor-independent. With the projection formula and functoriality, we now obtain the following natural sequence of quasi-isomorphisms:

The result follows.

$$
\begin{aligned}
\mathrm{R}\left(f_{\mathrm{qc}}\right)_{*}\left(\mathcal{M} \otimes_{\mathcal{O}_{X}}^{\mathrm{L}} \mathrm{L} f_{\mathrm{qc}}^{*} \mathrm{R}\left(g_{\mathrm{qc}}\right)_{*} \mathcal{O}_{Y^{\prime}}\right) & \simeq \mathrm{R}\left(f_{\mathrm{qc}}\right)_{*}\left(\mathcal{M} \otimes_{\mathcal{O}_{X}}^{\mathrm{L}} \mathrm{R}\left(g_{\mathrm{qc}}^{\prime}\right)_{*} \mathcal{O}_{X^{\prime}}\right) \\
& \simeq \mathrm{R}\left(f_{\mathrm{qc}}\right)_{*} \mathrm{R}\left(g_{\mathrm{qc}}^{\prime}\right)_{*} \mathrm{~L} g_{\mathrm{qc}}^{\prime *} \mathcal{M} \\
& \simeq \mathrm{R}\left(g_{\mathrm{qc}}\right)_{*} \mathrm{R}\left(f_{\mathrm{qc}}^{\prime}\right)_{*} \mathrm{~L} g_{\mathrm{qc}}^{\prime *} \mathcal{M} .
\end{aligned}
$$

Note that in the setting of derived algebraic geometry, tor-independence is not necessary to obtain a base change result [BZFN10, Prop. 3.10].

4.6. Finite duality. Using the projection formula, we can also establish finite duality.

Theorem 4.14. Let $f: X \rightarrow Y$ be a concentrated morphism of algebraic stacks.

(1) $\mathrm{R}\left(f_{\mathrm{qc}}\right)_{*}: \mathrm{D}_{\mathrm{qc}}(X) \rightarrow \mathrm{D}_{\mathrm{qc}}(Y)$ admits a right adjoint $f^{\times}$.

(2) For every $\mathcal{M} \in \mathrm{D}_{\mathrm{qc}}(Y)$ there is a natural quasi-isomorphism:

$$
\mathrm{R}\left(f_{\mathrm{qc}}\right)_{*} f^{\times}(\mathcal{M}) \simeq \mathrm{R} \mathcal{H} \operatorname{com}_{\mathcal{O}_{Y}}^{\mathrm{qc}}\left(\mathrm{R}\left(f_{\mathrm{qc}}\right)_{*} \mathcal{O}_{X}, \mathcal{M}\right)
$$

(3) If $f$ is affine, then for every $\mathcal{M} \in \mathrm{D}_{\mathrm{qc}}(Y)$ there is a natural quasi-isomorphism:

$$
f^{\times}(\mathcal{M}) \simeq \bar{f}^{*} \mathrm{R} \mathcal{H} \operatorname{com}_{\mathcal{O}_{Y}}^{\mathrm{qc}}\left(f_{*} \mathcal{O}_{X}, \mathcal{M}\right),
$$

where $\bar{f}^{*}$ is the functor from Corollary 2. 7 .

(4) If $f$ is affine and $f_{*} \mathcal{O}_{X}$ is perfect, then for every $\mathcal{M} \in \mathrm{D}_{\mathrm{qc}}(Y)$ there is a natural quasi-isomorphism:

$$
f^{\times}\left(\mathcal{O}_{Y}\right) \otimes_{\mathcal{O}_{X}}^{\mathrm{L}} f^{*}(\mathcal{M}) \simeq f^{\times}(\mathcal{M}) .
$$

In particular, $f^{\times}$preserves small coproducts. Moreover, $f^{\times}$is compatible with tor-independent base change on $Y$. If in addition $f$ is surjective, then $f^{\times}$is conservative.

Proof. By Theorem 2.6 3), the functor $\mathrm{R}\left(f_{\mathrm{qc}}\right)_{*}$ preserves small coproducts. Since $\mathrm{D}_{\mathrm{qc}}(X)$ is well generated [HNR14, Thm. B.1], the existence of $f^{\times}$follows from Nee01b, Prop. 1.20]. Now fix $\mathcal{N} \in \mathrm{D}_{\mathrm{qc}}(Y)$; then there are natural isomorphisms:

$$
\begin{aligned}
\operatorname{Hom}_{\mathcal{O}_{Y}}\left(\mathcal{N}, \mathrm{R}\left(f_{\mathrm{qc}}\right)_{*} f^{\times}(\mathcal{M})\right) & \cong \operatorname{Hom}_{\mathcal{O}_{X}}\left(\mathrm{~L} f_{\mathrm{qc}}^{*} \mathcal{N}, f^{\times}(\mathcal{M})\right) \\
& \cong \operatorname{Hom}_{\mathcal{O}_{Y}}\left(\mathrm{R}\left(f_{\mathrm{qc}}\right)_{*} \mathrm{~L} f_{\mathrm{qc}}^{*} \mathcal{N}, \mathcal{M}\right) \\
& \cong \operatorname{Hom}_{\mathcal{O}_{Y}}\left(\left(\mathrm{R}\left(f_{\mathrm{qc}}\right)_{*} \mathcal{O}_{X}\right) \otimes_{\mathcal{O}_{Y}}^{\mathrm{L}} \mathcal{N}, \mathcal{M}\right) \\
& \cong \operatorname{Hom}_{\mathcal{O}_{Y}}\left(\mathcal{N}, \mathrm{R} \mathcal{H} \operatorname{Hom}_{\mathcal{O}_{Y}}^{\mathrm{qc}}\left(\mathrm{R}\left(f_{\mathrm{qc}}\right)_{*} \mathcal{O}_{X}, \mathcal{M}\right)\right)
\end{aligned}
$$

The penultimate isomorphism follows from the projection formula (Corollary 4.12). By the Yoneda Lemma, this proves (2).

We now address (3). Let $\tilde{f}^{\times}: \mathrm{D}_{\mathrm{qc}}(Y) \rightarrow \mathrm{D}_{\mathrm{qc}}(X)$ be the functor

$$
\tilde{f}^{\times}(\mathcal{M})=\bar{f}^{*} \mathrm{R} \mathcal{H} \operatorname{Com}_{\mathcal{O}_{Y}}^{\mathrm{qc}}\left(f_{*} \mathcal{O}_{X}, \mathcal{M}\right),
$$

where $\bar{f}^{*}$ comes from the equivalence of Corollary 2.7. We claim there is a natural transformation of functors $\tilde{f}^{\times} \Rightarrow f^{\times}$. To see this, let $\mathcal{N} \in \mathrm{D}_{\mathrm{qc}}(X)$ and $\mathcal{M} \in \mathrm{D}_{\mathrm{qc}}(Y)$; then there are natural morphisms:

$$
\begin{aligned}
& \operatorname{Hom}_{\mathcal{O}_{X}}\left(\mathcal{N}, \tilde{f}^{\times}(\mathcal{M})\right) \rightarrow \operatorname{Hom}_{\mathcal{O}_{Y}}\left(\mathrm{R}\left(f_{\mathrm{qc}}\right)_{*} \mathcal{N}, \mathrm{R}\left(f_{\mathrm{qc}}\right)_{*} \tilde{f}^{\times}(\mathcal{M})\right) \\
& =\operatorname{Hom}_{\mathcal{O}_{Y}}\left(\mathrm{R}\left(f_{\mathrm{qc}}\right)_{*} \mathcal{N}, \mathrm{R} \mathcal{H} \operatorname{com}_{\mathcal{O}_{Y}}^{\mathrm{qc}}\left(f_{*} \mathcal{O}_{X}, \mathcal{M}\right)\right) \\
& \rightarrow \operatorname{Hom}_{\mathcal{O}_{Y}}\left(\mathrm{R}\left(f_{\mathrm{qc}}\right)_{*} \mathcal{N}, \mathrm{RH} \operatorname{com}_{\mathcal{O}_{Y}}^{\mathrm{qc}}\left(\mathcal{O}_{Y}, \mathcal{M}\right)\right) \\
& \cong \operatorname{Hom}_{\mathcal{O}_{Y}}\left(\mathrm{R}\left(f_{\mathrm{qc}}\right)_{*} \mathcal{N}, \mathcal{M}\right) \\
& \cong \operatorname{Hom}_{\mathcal{O}_{X}}\left(\mathcal{N}, f^{\times}(\mathcal{M})\right) \text {. }
\end{aligned}
$$


By the Yoneda Lemma, we have the claim. Since $f$ is affine, to prove that the natural transformation $\tilde{f}^{\times} \Rightarrow f^{\times}$is an isomorphism, it is sufficient to prove it is after application of $\mathrm{R}\left(f_{\mathrm{qc}}\right)_{*}$ (Lemma 2.8). This follows from the definition of $\tilde{f}^{\times}$ and (2).

We now treat (4). By adjunction and the projection formula (Corollary 4.12) we obtain a natural morphism for every $\mathcal{M} \in \mathrm{D}_{\mathrm{qc}}(Y)$ :

$$
f^{\times}\left(\mathcal{O}_{Y}\right) \otimes_{\mathcal{O}_{X}}^{\mathrm{L}} \mathrm{L} f_{\mathrm{qc}}^{*}(\mathcal{M}) \rightarrow f^{\times}(\mathcal{M}) .
$$

Since $f$ is affine, it is sufficient to prove this morphism is an isomorphism after application of $\mathrm{R}\left(f_{\mathrm{qc}}\right)_{*}$ (Lemma 2.8). By (2) and the projection formula (Corollary 4.12), we see that it is sufficient to prove that the induced morphism:

$$
\mathrm{RH}_{\mathcal{H}} m_{\mathcal{O}_{Y}}^{\mathrm{qc}}\left(f_{*} \mathcal{O}_{X}, \mathcal{O}_{Y}\right) \otimes_{\mathcal{O}_{Y}}^{\mathrm{L}} \mathcal{M} \rightarrow \mathrm{RH} \operatorname{com}_{\mathcal{O}_{Y}}^{\mathrm{qc}}\left(f_{*} \mathcal{O}_{X}, \mathcal{M}\right)
$$

is a quasi-isomorphism for every $\mathcal{M} \in \mathrm{D}_{\mathrm{qc}}(Y)$. But $f_{*} \mathcal{O}_{X}$ is perfect, so Lemma 4.32 now gives the claim.

For the compatibility of $f^{\times}$with base change, we consider a tor-independent 2-cartesian diagram of algebraic stacks:

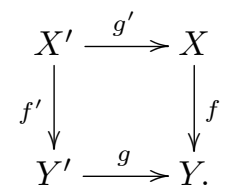

Adjointness and tor-independent base change (Corollary 4.13 provides a natural transformation $\mathrm{L} g_{\mathrm{qc}}^{\prime *} f^{\times} \rightarrow\left(f^{\prime}\right)^{\times} \mathrm{L} g_{\mathrm{qc}}^{*}$ of functors that we must show is an isomorphism. Tor-independent base change also implies that there is a quasi-isomorphism: $\mathrm{L} g_{\mathrm{qc}}^{*} \mathrm{R}\left(f_{\mathrm{qc}}\right)_{*} \mathcal{O}_{X} \simeq \mathrm{R}\left(f_{\mathrm{qc}}^{\prime}\right)_{*} \mathrm{~L} g_{\mathrm{qc}}^{\prime *} \mathcal{O}_{X}$. Since $\mathrm{R}\left(f_{\mathrm{qc}}\right)_{*} \mathcal{O}_{X}$ is perfect, it follows that $\mathrm{R}\left(f_{\mathrm{qc}}^{\prime}\right)_{*} \mathcal{O}_{X^{\prime}}$ is perfect. By the formula just determined for $f^{\times}$, we thus see that it is sufficient to prove that $\mathrm{L} g_{\mathrm{qc}}^{*} f^{\times}\left(\mathcal{O}_{Y}\right) \rightarrow\left(f^{\prime}\right)^{\times} \mathrm{L} g_{\mathrm{qc}}^{*}\left(\mathcal{O}_{Y}\right)$ is a quasi-isomorphism. Since $f$ (and so $f^{\prime}$ ) is affine, it is sufficient to verify this after application of $\mathrm{R}\left(f_{\mathrm{qc}}^{\prime}\right)_{*}$. This observation, together with (2) and tor-independent base change shows that it is sufficient to prove that the morphism:

$$
\mathrm{L} g_{\mathrm{qc}}^{*} \mathrm{R} \mathcal{H} \mathcal{C o m}_{\mathcal{O}_{Y}}^{\mathrm{qc}}\left(f_{*} \mathcal{O}_{X}, \mathcal{O}_{Y}\right) \rightarrow \mathrm{R} \mathcal{H} \operatorname{Com}_{\mathcal{O}_{Y^{\prime}}}\left(\mathrm{L}_{\mathrm{qc}}^{*}\left(f_{*} \mathcal{O}_{X}\right), \mathcal{O}_{Y^{\prime}}\right)
$$

is a quasi-isomorphism. But $f_{*} \mathcal{O}_{X}$ and $\mathrm{L} g_{\mathrm{qc}}^{*}\left(f_{*} \mathcal{O}_{X}\right)$ are both perfect and so dualizable (Lemma 4.3). In particular, the derived pullback of the dual of $f_{*} \mathcal{O}_{X}$ coincides with the dual of $\mathrm{L} g_{\mathrm{qc}}^{*}\left(f_{*} \mathcal{O}_{X}\right)$. It follows that the asserted map is a quasiisomorphism and the claim follows.

Finally, we address the conservativity. For this, it is sufficient to observe that if $f$ is surjective, then $\operatorname{supph}\left(f_{*} \mathcal{O}_{X}\right)=|Y|$. But $\mathrm{RH} \mathcal{H o m}_{\mathcal{O}_{Y}}\left(f_{*} \mathcal{O}_{X}, \mathcal{M}\right) \simeq 0$ if and only if $\mathcal{M} \simeq 0$ (Lemma 4.9).

Corollary 4.15. If $f: X \rightarrow Y$ is a finite and faithfully flat morphism of finite presentation between algebraic stacks, then the functor

$$
f^{\times}(\mathcal{M})=\bar{f}^{*} \mathrm{R} \mathcal{H} \operatorname{Hom}_{\mathcal{O}_{Y}}\left(f_{*} \mathcal{O}_{X}, \mathcal{M}\right), \quad \text { where } \mathcal{M} \in \mathrm{D}_{\mathrm{qc}}(Y),
$$

is right adjoint to $\mathrm{R}\left(f_{\mathrm{qc}}\right)_{*}: \mathrm{D}_{\mathrm{qc}}(X) \rightarrow \mathrm{D}_{\mathrm{qc}}(Y)$. Moreover, $f^{\times}$is compatible with arbitrary base change on $Y, f^{\times}\left(\mathcal{O}_{Y}\right) \otimes_{\mathcal{O}_{X}}^{\llcorner} f^{*}(-) \simeq f^{\times}(-)$, preserves small coproducts, and is conservative.

4.7. Coherent functors. Combining the strong projection formula of Proposition 4.11 with the characterization of compact objects in Lemma 4.5 , we can prove most of Theorem D, 
Corollary 4.16. Let $A$ be a noetherian ring and let $\pi: X \rightarrow \operatorname{Spec} A$ be a morphism of finite type between noetherian algebraic stacks. Suppose that

(1) for every $i \geq 0$ and $\mathcal{M} \in \operatorname{Coh}(X)$, the cohomology $H^{i}\left(X_{\text {lis-ét }}, \mathcal{M}\right)$ is a finitely generated A-module (e.g., $\pi$ proper); and

(2) $\mathrm{D}_{\mathrm{qc}}(X)$ is compactly generated.

Then, for every $\mathcal{F} \in \mathrm{D}_{\mathrm{qc}}(X)$ and $\mathcal{G} \in \mathrm{D}_{\mathrm{Coh}}^{b}(X)$, the functor

$$
\operatorname{Hom}_{\mathcal{O}_{X}}\left(\mathcal{F}, \mathcal{G} \otimes_{\mathcal{O}_{X}}^{\mathrm{L}} \mathrm{L} \pi_{\mathrm{qc}}^{*}(-)\right): \operatorname{Mod}(A) \rightarrow \operatorname{Mod}(A)
$$

is coherent.

Proof. We begin by observing that the coherent functors $\operatorname{Mod}(A) \rightarrow \operatorname{Mod}(A)$ constitute a full abelian subcategory of the category of $A$-linear functors, which is closed under products (where everything is computed "pointwise") [Hal14, Ex. 4.9]. Let $\mathcal{T} \subseteq \mathrm{D}_{\mathrm{qc}}(X)$ denote the full subcategory with objects those $\mathcal{F} \in \mathrm{D}_{\mathrm{qc}}(X)$ where the functor $\operatorname{Hom}_{\mathcal{O}_{X}}\left(\mathcal{F}, \mathcal{G} \otimes_{\mathcal{O}_{X}}^{\llcorner} \mathrm{L} \pi_{\mathrm{qc}}^{*}(-)\right)$ is coherent for every $\mathcal{G} \in \mathrm{D}_{\text {Coh }}^{b}(X)$. In particular, $\mathcal{T}$ is closed under small coproducts, shifts, and triangles. By Corollary 3.14, it is enough to prove that $\mathcal{T}$ contains the compact objects of $\mathrm{D}_{\mathrm{qc}}(X)$. If $Q \in \mathrm{D}_{\mathrm{qc}}(X)$ is compact, then the strong projection formula (Proposition 4.11) implies that there is a natural quasi-isomorphism:

$$
\operatorname{RHom}_{\mathcal{O}_{X}}(Q, \mathcal{G}) \otimes_{A}^{\mathrm{L}} I \simeq \operatorname{RHom}_{\mathcal{O}_{X}}\left(Q, \mathcal{G} \otimes_{\mathcal{O}_{X}}^{\mathrm{L}} \mathrm{L} \pi_{\mathrm{qc}}^{*} I\right) .
$$

Since $\mathcal{Q}$ is compact, it is perfect (Lemma 4.4 11 ) and so $\operatorname{RH} \operatorname{Hom}_{\mathcal{O}_{X}}(\mathcal{Q}, \mathcal{G}) \in \mathrm{D}_{\text {Coh }}^{b}(X)$. The assumption on preservation of coherence implies that $\mathrm{R}\left(\pi_{\mathrm{qc}}\right)_{*}$ sends $\mathrm{D}_{\text {Coh }}^{+}(X)$ to $\mathrm{D}_{\text {Coh }}^{+}(A)$. In particular, $\operatorname{RHom}_{\mathcal{O}_{X}}(Q, \mathcal{G}) \simeq \mathrm{R}\left(\pi_{\mathrm{qc}}\right)_{*} \mathrm{RH} \mathcal{H}_{\mathcal{O}_{X}}(\mathcal{Q}, \mathcal{G}) \in \mathrm{D}_{\text {Coh }}^{+}(A)$. By Lemma 4.5 we also have $\operatorname{RHom}_{\mathcal{O}_{X}}(\mathcal{Q}, \mathcal{G}) \in \mathrm{D}^{b}(A)$. Thus the functor $\operatorname{Hom}_{\mathcal{O}_{X}}\left(\mathcal{Q}, \mathcal{G} \otimes_{\mathcal{O}_{X}}^{\llcorner}\right.$ $\left.\mathrm{L} \pi_{\mathrm{qc}}^{*}(-)\right)$ is coherent [Hal14, Ex. 4.13] and we deduce the result.

\section{Presheaves of triangulated Categories}

Throughout this section we fix a small category $\mathcal{D}$ that admits all finite limits. Let TCat denote the 2-category of triangulated categories. A $\mathcal{D}$-presheaf of triangulated categories is a 2 -functor $\mathcal{T}: \mathcal{D}^{\circ} \rightarrow$ TCat.

Given a morphism $f: U \rightarrow V$ in $\mathcal{D}$, there is an induced pullback functor $f_{\mathcal{T}}^{*}: \mathcal{T}(V) \rightarrow$ $\mathcal{T}(U)$. When there is no cause for confusion, we will suppress the subscript $\mathcal{T}$ from $f_{\mathcal{T}}^{*}$. For any such $f$ (not necessarily a monomorphism), we let

$$
\mathcal{T}_{V \backslash U}(V)=\operatorname{ker}\left(f^{*}: \mathcal{T}(V) \rightarrow \mathcal{T}(U)\right) .
$$

We say that $\mathcal{T}$ has adjoints if for every morphism $f: U \rightarrow V$ in $\mathcal{D}$, the pullback functor $f^{*}: \mathcal{T}(V) \rightarrow \mathcal{T}(U)$ admits a right adjoint $f_{*}: \mathcal{T}(U) \rightarrow \mathcal{T}(V)$.

Definition 5.1. Suppose that $\mathcal{T}$ is a $\mathcal{D}$-presheaf of triangulated categories with adjoints. Let $f: U \rightarrow V$ be a morphism in $\mathcal{D}$ and let $N \in \mathcal{T}(V)$. We denote by $\eta_{N}^{f}: N \rightarrow f_{*} f^{*} N$ the unit of the adjunction. A morphism $g: W \rightarrow V$ in $\mathcal{D}$ is $\mathcal{T}$-preflat if for every cartesian square in $\mathcal{D}$ :

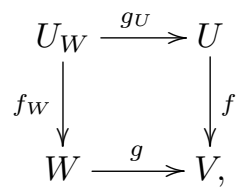

the natural transformation $g^{*} f_{*} \rightarrow\left(f_{W}\right)_{*}\left(g_{U}\right)^{*}$ is an isomorphism. A morphism $g: W \rightarrow V$ in $\mathcal{D}$ is $\mathcal{T}$-flat if for every morphism $V^{\prime} \rightarrow V$, the pullback $g^{\prime}: W^{\prime} \rightarrow V^{\prime}$ of $g$ is $\mathcal{T}$-preflat.

Note that because monomorphisms are stable under base change, $\mathcal{T}$-flat monomorphisms are stable under base change. 
Example 5.2. Let $Y$ be an algebraic stack that is quasi-compact and quasiseparated. Let $\boldsymbol{R e p}^{\mathrm{fp}} / Y$ denote the category of 1-morphisms $X \rightarrow Y$ that are representable and of finite presentation. The category $\boldsymbol{R e p}^{\mathrm{fp}} / Y$ is small. We have a $\boldsymbol{R e p}^{\mathrm{fp}} / Y$-presheaf of triangulated categories $\mathrm{D}_{\mathrm{qc}}:\left(\mathbf{R e p}^{\mathrm{fp}} / Y\right)^{\circ} \rightarrow$ TCat, that sends $X \rightarrow Y$ to $\mathrm{D}_{\mathrm{qc}}(X)$ and a 1-morphism $f: X^{\prime} \rightarrow X$ in $\mathbf{R e p}^{\mathrm{fp}} / Y$ to $\mathrm{L} f_{\mathrm{qc}}^{*}: \mathrm{D}_{\mathrm{qc}}(X) \rightarrow \mathrm{D}_{\mathrm{qc}}\left(X^{\prime}\right)$. The functor $\mathrm{L} f_{\mathrm{qc}}^{*}$ admits a right adjoint $\mathrm{R}\left(f_{\mathrm{qc}}\right)_{*}$ so $\mathrm{D}_{\mathrm{qc}}$ is a presheaf with adjoints.

By Theorem 2.6(4), if $f$ is a flat morphism, then it is $\mathrm{D}_{\mathrm{qc}}$-flat. Conversely, if $f: X^{\prime} \rightarrow X$ is $\mathrm{D}_{\mathrm{qc}}$-flat, then $f$ is flat. Indeed, this is local on the source and target of $f$, so it is sufficient to show that if $f: \operatorname{Spec} B \rightarrow \operatorname{Spec} A$ is $\mathrm{D}_{\mathrm{qc}}$-preflat, then $B$ is a flat $A$-algebra. For this, we note that if $I$ is an ideal of $A$, then corresponding to $i: \operatorname{Spec}(A / I) \rightarrow \operatorname{Spec} A$ we see that there is a quasi-isomorphism $(A / I) \otimes_{A}^{\mathrm{L}} B \simeq$ $(B / I B)[0]$. That is, for all $n>0$ and ideals $I$ of $A$ we have that $\operatorname{Tor}_{A}^{n}(B, A / I)=0$ hence $B$ is flat over $A$. It follows that the $\mathrm{D}_{\mathrm{qc}}$-flat monomorphisms are the quasicompact open immersions [EGA, IV.17.9.1].

Example 5.3. Our notion of $\mathcal{T}$-flatness is not always optimal. In particular, it is weaker than expected in the derived setting. If $\mathcal{T}$ is a presheaf of triangulated categories with $t$-structures, then a better definition is that $f$ is $\mathcal{T}$-flat if $f^{*}$ is $t$-exact.

To illustrate this, suppose $\mathcal{D}=\mathrm{SCR}^{\circ}$ is the $\infty$-category of affine derived schemes, that is, the opposite category to the $\infty$-category of simplicial commutative rings. Further, let $\mathcal{T}=\operatorname{Mod}(-)$ be the functor that takes a simplicial commutative ring $A$ to the stable $\infty$-category $\operatorname{Mod}(A)$ of (not necessarily connective) $A$-modules. Then every morphism in $\mathcal{D}$ is $\mathcal{T}$-flat whereas $\operatorname{Spec} B \rightarrow \operatorname{Spec} A$ is flat exactly when the pullback $B \stackrel{\mathrm{L}}{\otimes} A-: \operatorname{Mod}(A) \rightarrow \operatorname{Mod}(B)$ is $t$-exact. Nevertheless, just as in the non-derived case, the finitely presented $\mathcal{T}$-flat monomorphisms are exactly the quasi-compact open immersions since every monomorphism of derived schemes is formally étale [HAGII, 2.2.2.5 (2)].

Lemma 5.4. Let $\mathcal{T}$ be a $\mathcal{D}$-presheaf of triangulated categories with adjoints. Fix a commutative diagram in $\mathcal{D}$ :

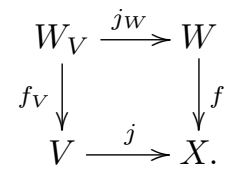

(1) If $j$ is a $\mathcal{T}$-preflat monomorphism in $\mathcal{D}$, then the adjunction $j^{*} j_{*} \rightarrow \operatorname{Id}_{\mathcal{T}(V)}$ is an isomorphism.

(2) $f^{*}: \mathcal{T}_{X \backslash V}(X) \rightarrow \mathcal{T}(W)$ factors through $\mathcal{T}_{W \backslash W_{V}}(W)$.

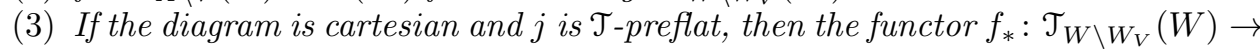
$\mathcal{T}(X)$ factors through $\mathcal{T}_{X \backslash V}(X)$ and is right adjoint to $f^{*}: \mathcal{T}_{X \backslash V}(X) \rightarrow$ $\mathcal{T}_{W \backslash W_{V}}(W)$.

Proof. If $j: V \rightarrow X$ is a monomorphism, then the commutative diagram:

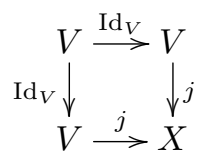

is cartesian, whence $j^{*} j_{*} \simeq\left(\operatorname{Id}_{V}\right)_{*}\left(\operatorname{Id}_{V}\right)^{*} \simeq \operatorname{Id}_{\mathcal{T}(V)}$.

By functoriality (2) is trivial. For (3), given $M \in \mathcal{T}_{W \backslash W_{V}}(W)$, then $j^{*} f_{*} M \simeq$ $\left(f_{V}\right)_{*}\left(j_{W}\right)^{*} M \simeq 0$, hence $f_{*} M \in \mathcal{T}_{X \backslash V}(X)$. 
Definition 5.5. Fix a $\mathcal{D}$-presheaf of triangulated categories $\mathcal{T}$ with adjoints. A Mayer-Vietoris $\mathcal{T}$-square is a cartesian diagram in $\mathcal{D}$ :

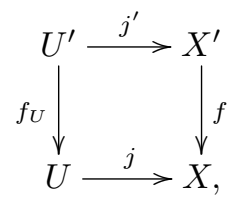

satisfying the following three conditions:

(1) $j$ is a $\mathcal{T}$-flat monomorphism,

(2) the natural transformation $f^{*} j_{*} \rightarrow j_{*}^{\prime} f_{U}^{*}$ is an isomorphism, and

(3) the induced functor $f^{*}: \mathcal{T}_{X \backslash U}(X) \rightarrow \mathcal{T}_{X^{\prime} \backslash U^{\prime}}\left(X^{\prime}\right)$ is an equivalence of categories.

Condition (2) for a Mayer-Vietoris $\mathcal{T}$-square is satisfied if $f$ is a $\mathcal{T}$-(pre)flat morphism. By tor-independent base change (Corollary 4.13), if $\mathcal{T}=\mathrm{D}_{\mathrm{qc}}$, then condition (2) is satisfied for every $f$. In [HR16, we will consider applications of these Mayer-Vietoris triangles to a result of Moret-Bailly MB96. For this intended application, it is essential that we permit $f$ to be non-flat.

Example 5.6. We continue with Example 5.2 Let $f: X^{\prime} \rightarrow X$ be a representable, quasi-compact, and quasi-separated étale neighborhood of a closed subset $|Z| \subseteq|X|$ with quasi-compact complement $|U|$. Let $j: U \hookrightarrow X$ be the resulting quasi-compact open immersion. Then the cartesian square:

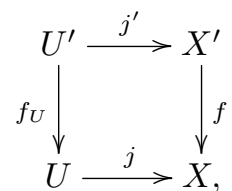

is a Mayer-Vietoris $\mathrm{D}_{\mathrm{qc}}$-square. To see this, it remains to prove that the functor $\mathrm{L} f_{\mathrm{qc}}^{*}$ induces the desired equivalence. Now the exact functor $f^{*}: \operatorname{Mod}(X) \rightarrow \operatorname{Mod}\left(X^{\prime}\right)$ admits an exact left adjoint $f_{!}: \operatorname{Mod}\left(X^{\prime}\right) \rightarrow \operatorname{Mod}(X)$ [Stacks, 03DI]. Explicitly, for $M \in \operatorname{Mod}\left(X^{\prime}\right)$ we have that $f_{!} M$ is the sheafification of the presheaf

$$
(V \rightarrow X) \underset{\phi \in \operatorname{Hom}_{X}\left(V, X^{\prime}\right)}{\bigoplus} M\left(V \stackrel{\phi}{\rightarrow} X^{\prime}\right) .
$$

Note that the natural map $M \rightarrow f^{*} f_{!} M$ is an isomorphism for all $M \in \operatorname{Mod}\left(X^{\prime}\right)$ such that $j^{\prime *} M=0$. Also, if $N \in \operatorname{Mod}(X)$ and $j^{*} N=0$, then the natural map $f_{!} f^{*} N \rightarrow N$ is an isomorphism. The exactness of the adjoint pair $\left(f_{!}, f^{*}\right)$ now gives an adjoint pair on the level of derived categories $\left(f_{!}, f^{*}\right): \mathrm{D}(X) \leftrightarrows \mathrm{D}\left(X^{\prime}\right)$ and that the relations just given also hold on the derived category. Next, we observe that the restriction of $f^{*}$ to $\mathrm{D}_{\mathrm{qc}}(X)$ coincides with $\mathrm{L} f_{\mathrm{qc}}^{*}$. Thus it remains to prove that if $M \in \mathrm{D}_{\mathrm{qc}}\left(X^{\prime}\right)$ and $\mathrm{L}\left(j^{\prime}\right)_{\mathrm{qc}}^{*} M \simeq 0$, then $f_{!} M \in \mathrm{D}_{\mathrm{qc}}(X)$. The exactness of $f_{!}$ and $\mathrm{L}\left(j^{\prime}\right)_{\mathrm{qc}}^{*}$ show that is sufficient to prove this result when $M$ is a quasi-coherent sheaf such that $\left(j^{\prime}\right)^{*} M=0$. Note that $\left(U \stackrel{j}{\rightarrow} X, X^{\prime} \stackrel{f}{\rightarrow} X\right)$ is an étale cover of $X$, $j^{*} f_{!} M=\left(f_{U}\right)_{!}\left(j^{\prime}\right)^{*} M=0$ and $f^{*} f_{!} M \cong M$. We deduce that étale-locally $f_{!} M$ is quasi-coherent. By descent $f_{!} M$ is quasi-coherent and the result is proved. For a different proof in a more general context, see [HR16, Prop. 4.2].

Example 5.7 (Étale cohomology). Let $Y$ be an algebraic stack that is quasicompact and quasi-separated. Let $\Lambda$ be a noetherian ring such that $\Lambda$ is torsion and $|\Lambda|$ is invertible on $Y$. We have the derived category $\mathrm{D}_{\text {cart }}^{+}(Y, \Lambda)$ of bounded below 
lisse-étale $\Lambda$-modules on $Y$ with cartesian cohomology LMB, 12.10]. More generally, we have a $\boldsymbol{R e p}^{\mathrm{fp}} / Y$-presheaf of triangulated categories $\mathrm{D}_{\text {cart }}^{+}(-, \Lambda):\left(\boldsymbol{R e p}^{\mathrm{fp}} / Y\right)^{\circ} \rightarrow$ TCat, that sends $X \rightarrow Y$ to $\mathrm{D}_{\text {cart }}^{+}(X, \Lambda)$ and a 1-morphism $f: X^{\prime} \rightarrow X$ in $\operatorname{Rep}^{\mathrm{fp}} / Y$ to $f^{*}: \mathrm{D}_{\text {cart }}^{+}(X, \Lambda) \rightarrow \mathrm{D}_{\text {cart }}^{+}\left(X^{\prime}, \Lambda\right)$ Ols07, 9.16]. By smooth base change, $f_{*}$ takes cartesian sheaves to cartesian sheaves so the functor $f^{*}$ admits a right adjoint $\mathrm{R} f_{*}$ Ols07, Prop. 9.9], i.e., $\mathrm{D}_{\text {cart }}^{+}(-, \Lambda)$ is a presheaf with adjoints. Moreover, étale and smooth morphisms are $\mathrm{D}_{\text {cart }}^{+}(-, \Lambda)$-flat.

If $f: X^{\prime} \rightarrow X$ is an étale neighborhood of $|Z| \subseteq|X|$ as in the previous example, then the resulting square is a Mayer-Vietoris $\mathrm{D}_{\text {cart }}^{+}(-, \Lambda)$-square. Indeed, if $i: Z \rightarrow$ $X$ is a closed immersion for some scheme structure on $Z$, then $i^{*}$ and $i_{*}$ induce an equivalence of categories $\mathrm{D}_{\text {cart },|X \backslash U|}^{+}(X, \Lambda) \cong \mathrm{D}_{\text {cart }}^{+}(Z, \Lambda)$.

We also have a subpresheaf $\mathrm{D}_{\mathrm{c}}^{+}(-, \Lambda)$ where $\mathrm{D}_{\mathrm{c}}^{+}(X, \Lambda) \subseteq \mathrm{D}_{\text {cart }}^{+}(X, \Lambda)$ consists of the objects with constructible cohomology sheaves [LMB, 18.6]. If $Y$ is quasiexcellent of finite dimension, $\Lambda=\mathbb{Z} / N \mathbb{Z}$ and $N$ is invertible on $Y$, then $\mathrm{D}_{\mathrm{c}}^{+}(-, \Lambda)$ has adjoints (Deligne-Gabber's finiteness theorem) and an étale neighborhood is a Mayer-Vietoris $\mathrm{D}_{\mathrm{c}}^{+}(-, \Lambda)$-square.

These results are also valid for unbounded derived categories [LZ12, 6.3.3-6.3.4].

Example 5.8. Consider a 2-commutative diagram of triangulated functors:

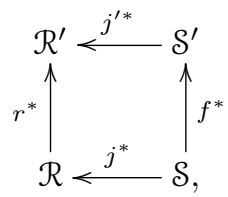

and assume that they all have right adjoints, which we will denote as $f_{*}, j_{*}, r_{*}$, and $j_{*}^{\prime}$, respectively. Let $\mathcal{K}=\operatorname{ker}\left(j^{*}\right)$ and $\mathcal{K}^{\prime}=\operatorname{ker}\left(j^{*}\right)$. Let $\mathcal{D}$ be the category consisting of the following objects and arrows:

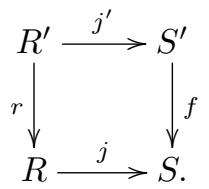

There is a $\mathcal{D}$-presheaf of triangulated categories $\mathcal{T}$ with adjoints such that $\mathcal{T}(S)=\mathcal{S}$ etc. The square above is a Mayer-Vietoris $\mathcal{T}$-square if and only if the following conditions are satisfied:

(1) the natural transformations $j^{*} j_{*} \rightarrow \operatorname{Id}$ and $j^{\prime *} j_{*}^{\prime} \rightarrow$ Id are isomorphisms,

(2) the natural transformation $j^{*} f_{*} \rightarrow r_{*} j^{* *}$ is an isomorphism,

(3) the natural transformation $f^{*} j_{*} \rightarrow j_{*}^{\prime} r^{*}$ is an isomorphism, and

(4) the induced functor $\mathcal{K} \rightarrow \mathcal{K}^{\prime}$ is an equivalence of categories.

Condition (1) implies that $\mathcal{R}=\mathcal{S} / \mathcal{K}, \mathcal{R}^{\prime}=\mathcal{S}^{\prime} / \mathcal{K}^{\prime}$ and $\mathcal{K}, \mathcal{K}^{\prime}$ are Bousfield subcategories of $\mathcal{S}, \mathcal{S}^{\prime}$ respectively (Lemma 3.4 and [Nee01b, Ch. 9]).

Mayer-Vietoris $\mathcal{T}$-squares give rise to many nice properties. In particular, we obtain a familiar distinguished triangle.

Lemma 5.9. Let $\mathcal{T}$ be a $\mathcal{D}$-presheaf of triangulated categories with adjoints. Consider a Mayer-Vietoris $\mathcal{T}$-square in $\mathcal{D}$ :

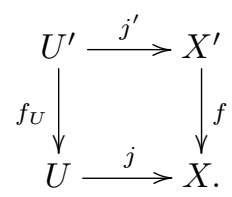


(1) If $N \in \mathcal{T}(X)$, then there is a unique map d that makes the triangle:

$$
N \stackrel{\left(\begin{array}{l}
\eta_{N}^{j} \\
\eta_{N}^{f}
\end{array}\right)}{\longrightarrow} j_{*} j^{*} N \oplus f_{*} f^{*} N \stackrel{\left(\eta_{j_{*} j^{*} N}^{f}-f_{*} f^{*} \eta_{N}^{j}\right)}{\longrightarrow} f_{*} f^{*} j_{*} j^{*} N \stackrel{d}{\longrightarrow} N[1]
$$

distinguished. Moreover, this d is functorial in $N$.

(2) Let $M \in \mathcal{T}_{X^{\prime} \backslash U^{\prime}}\left(X^{\prime}\right)$ and let $N \in \mathcal{T}(X)$. Then there is a natural bijection:

$$
\operatorname{Hom}_{\mathcal{T}(X)}\left(f_{*} M, N\right) \cong \operatorname{Hom}_{\mathcal{T}\left(X^{\prime}\right)}\left(M, f^{*} N\right) .
$$

(3) Given $N_{U} \in \mathcal{T}(U), N^{\prime} \in \mathcal{T}\left(X^{\prime}\right)$, and an isomorphism $\delta: j^{\prime *} N^{\prime} \rightarrow f_{U}^{*} N_{U}$, define $N$ by a distinguished triangle in $\mathcal{T}(X)$ :

$$
N \longrightarrow j_{*} N_{U} \oplus f_{*} N^{\prime} \stackrel{\left(\eta_{j_{*} N_{U}}^{f}-\alpha\right)}{\longrightarrow} f_{*} f^{*} j_{*} N_{U} \longrightarrow N[1],
$$

where $\alpha: f_{*} N^{\prime} \rightarrow f_{*} f^{*} j_{*} N_{U}$ is the composition:

$$
f_{*} N^{\prime} \stackrel{f_{*} \eta_{N^{\prime}}^{j^{\prime}}}{\longrightarrow} f_{*} j_{*}^{\prime} j^{\prime *} N^{\prime} \stackrel{f_{*} j_{*}^{\prime} \delta}{\longrightarrow} f_{*} j_{*}^{\prime} f_{U}^{*} N_{U} \cong f_{*} f^{*} j_{*} N_{U} .
$$

Then the induced maps $j^{*} N \rightarrow N_{U}$ and $f^{*} N \rightarrow N^{\prime}$ are isomorphisms.

(4) If $N \in \mathcal{T}(X)$ satisfies $j^{*} N \in \mathcal{T}(U)^{c}, f^{*} N \in \mathcal{T}\left(X^{\prime}\right)^{c}$, and $f_{U}^{*} j^{*} N \in \mathcal{T}\left(U^{\prime}\right)^{c}$, then $N \in \mathcal{T}(X)^{c}$.

Proof. An equivalent formulation of (1) is that

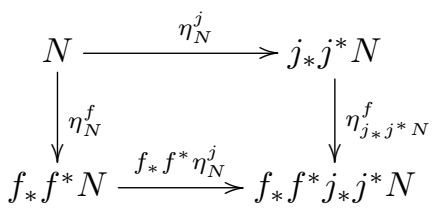

is a homotopy cartesian square [Nee01b, Def. 1.4.1] whose differential $d$ is unique and is functorial in $N$. To see that the square is cartesian, first choose $C$ such that we have a distinguished triangle:

$$
C \stackrel{l}{\longrightarrow} N \stackrel{\eta_{N}^{j}}{\longrightarrow} j_{*} j^{*} N \stackrel{m}{\longrightarrow} C[1] .
$$

Since $j$ is a $\mathcal{T}$-flat monomorphism, $j^{*} \eta_{N}^{j}$ is an isomorphism (Lemma 5.4 1]). It follows that $j^{*} C \cong 0$, so $C \in \mathcal{T}_{X \backslash U}(X)$, and $\eta_{C}^{f}$ is an isomorphism. We thus obtain a morphism of distinguished triangles:

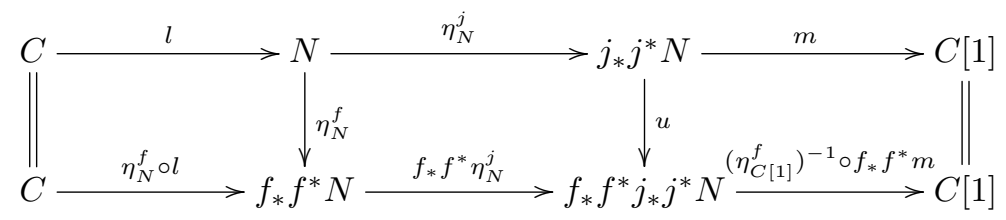

for some morphism $u$. We can certainly let $u=\eta_{j_{*} j^{*} N}^{f}$ and we will soon see that this is actually the only possible $u$. On the other hand, we can choose $u$ such that the middle square is a homotopy cartesian square by the Octahedral Axiom Nee01b, Lem. 1.4.3]. After applying $j_{*} j^{*}$ to the middle square and adjoining to it the natural square relating $u$ and $j_{*} j^{*} u$ we obtain the commutative diagram:

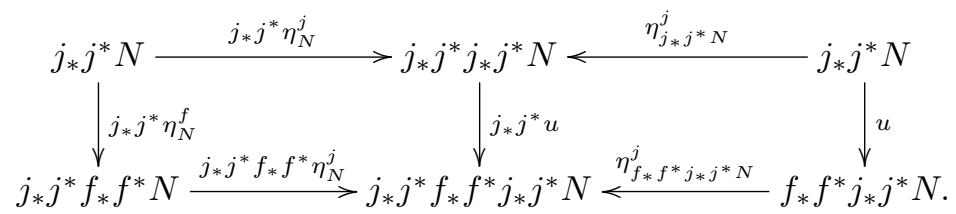


Since $j$ is a $\mathcal{T}$-flat monomorphism, the horizontal maps are all isomorphisms and it follows that $u=\eta_{j_{*} j^{*} N}^{f}$. Moreover, it is readily verified that the induced differential

$$
d:=l[1] \circ\left(\eta_{C}^{f}\right)^{-1}[1] \circ f_{*} f^{*} m: f_{*} f^{*} j_{*} j^{*} N \rightarrow N[1]
$$

is independent of the choice of the triangle $C \stackrel{l}{\rightarrow} N \stackrel{\eta_{N}^{j}}{\rightarrow} j_{*} j^{*} N \stackrel{m}{\rightarrow} C[1]$. The functoriality of the Mayer-Vietoris triangle now follows from the construction. Finally, to show that $d$ is unique, if $d^{\prime}: f_{*} f^{*} j_{*} j^{*} N \rightarrow N[1]$ is another morphism that makes a distinguished triangle, then there is an induced morphism of distinguished triangles:

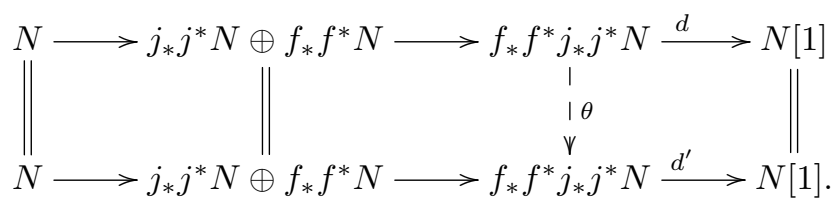

It remains to show that $\theta$ is the identity morphism. Splitting up the sum in the middle square, we obtain the commutative diagram:

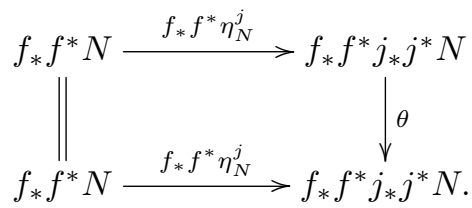

Applying $j_{*} j^{*}$ to this diagram, we may append another square on the right to obtain the commutative diagram:

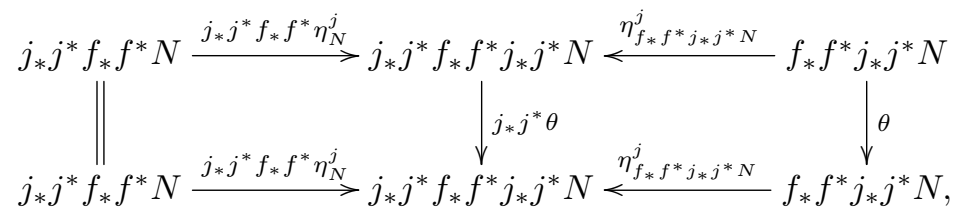

where the horizontal arrows are isomorphisms. It follows that $\theta$ is the identity.

To obtain the isomorphism in (2), first note that by the definition of a MayerVietoris square, the counit $f^{*} f_{*} M \rightarrow M$ is an isomorphism since $M \in \mathcal{T}_{X^{\prime} \backslash U^{\prime}}\left(X^{\prime}\right)$. This gives us a natural isomorphism

$$
\operatorname{Hom}_{\mathcal{T}\left(X^{\prime}\right)}\left(M, f^{*} N\right) \cong \operatorname{Hom}_{\mathcal{T}\left(X^{\prime}\right)}\left(f^{*} f_{*} M, f^{*} N\right) \cong \operatorname{Hom}_{\mathcal{T}(X)}\left(f_{*} M, f_{*} f^{*} N\right) .
$$

Now apply the homological functor $\operatorname{Hom}_{\mathcal{T}(X)}\left(f_{*} M,-\right)$ to the triangle $N \rightarrow j_{*} j^{*} N \oplus$ $f_{*} f^{*} N \rightarrow f_{*} f^{*} j_{*} j^{*} N$ from (1). Since $j^{*} f_{*} M \simeq 0$ we obtain an isomorphism $\operatorname{Hom}_{\mathcal{T}(X)}\left(f_{*} M, N\right) \cong \operatorname{Hom}_{\mathcal{T}(X)}\left(f_{*} M, f_{*} f^{*} N\right)$ and the result follows.

For (3), the natural maps $v_{j}: j^{*} N \rightarrow N_{U}$ and $v_{f}: f^{*} N \rightarrow N^{\prime}$ are obtained by adjunction from the maps $v_{j}^{\vee}: N \rightarrow j_{*} N_{U}$ and $v_{f}^{\vee}: N \rightarrow f_{*} N^{\prime}$ in the defining triangle of $N$. The defining triangle exhibits $N$ as a homotopy pullback. We may thus find a morphism of distinguished triangles [Nee01b, Lem. 1.4.4] (Octahedral axiom):

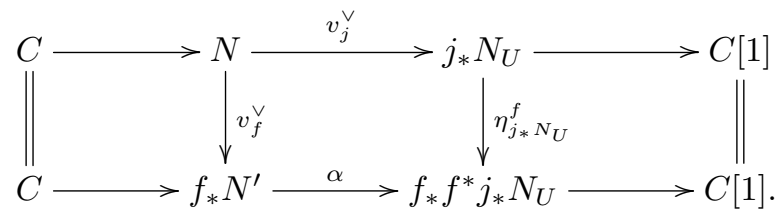

Since $j$ is a $\mathcal{T}$-flat monomorphism, we have that $j^{*} \alpha$ is an isomorphism, so $j^{*} C \cong 0$. It follows that $j^{*} v_{j}^{\vee}: j^{*} N \rightarrow j^{*} j_{*} N_{U}$ is an isomorphism and hence that $v_{j}: j^{*} N \rightarrow$ $N_{U}$ is an isomorphism (Lemma 5.4 1) ). 
Now, by (1), we have a morphism of distinguished triangles:

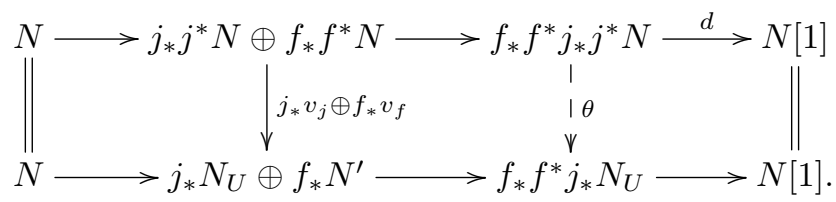

As before, it follows that $\theta=f_{*} f^{*} j_{*} v_{j}$ by considering the application of $j_{*} j^{*}$ to the middle square. Since $v_{j}$ is an isomorphism, it follows that $f_{*} v_{f}$ is an isomorphism. Now, if we let $W$ be a cone of $v_{f}$, then $f_{*} W \simeq 0$. We will be done if we can show that $j^{\prime *} v_{f}$ is an isomorphism. Indeed, it would then follow that $j^{\prime *} W \simeq 0$ and so $W \simeq f^{*} f_{*} W \simeq f^{*} 0 \simeq 0$. To this end, since the following diagram commutes:

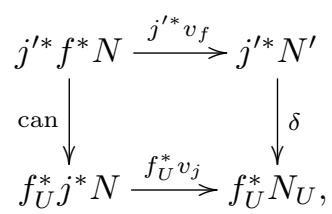

and all appearing morphisms except $j^{\prime *} v_{f}$ are known to be isomorphisms, it follows that $j^{\prime *} v_{f}$ is an isomorphism.

For (4), let $h \in\left\{j, f, j \circ f_{U}\right\}$. Since $h^{*}$ admits a right adjoint, it commutes with small coproducts. Thus if $\left\{Q_{\lambda}\right\}$ is a set of objects of $\mathcal{T}(X)$, then

$$
\begin{aligned}
\oplus_{\lambda} \operatorname{Hom}\left(N, h_{*} h^{*} Q_{\lambda}\right) & \cong \oplus_{\lambda} \operatorname{Hom}\left(h^{*} N, h^{*} Q_{\lambda}\right) \\
& \cong \operatorname{Hom}\left(h^{*} N, \oplus_{\lambda} h^{*} Q_{\lambda}\right) \\
& \cong \operatorname{Hom}\left(h^{*} N, h^{*}\left(\oplus_{\lambda} Q_{\lambda}\right)\right) \\
& \cong \operatorname{Hom}\left(N, h_{*} h^{*}\left(\oplus_{\lambda} Q_{\lambda}\right)\right) .
\end{aligned}
$$

The result now follows by consideration of the Mayer-Vietoris triangles associated to $Q_{\lambda}$ and $\oplus_{\lambda} Q_{\lambda}$, together with the long exact sequence given by the homological functor $\operatorname{Hom}(N,-)$.

In the following definition, we axiomatize the required properties of open immersions of algebraic stacks.

Definition 5.10. Let $\mathcal{T}$ be a $\mathcal{D}$-presheaf of triangulated categories with adjoints. Let $\mathcal{L}$ be a collection of morphisms in $\mathcal{D}$. We say that $\mathcal{L}$ supports $\mathcal{T}$ if it satisfies the following five conditions:

(1) if $j: U \rightarrow X$ belongs to $\mathcal{L}$, then $j$ is a $\mathcal{T}$-flat monomorphism;

(2) if $j: U \rightarrow V$ is an isomorphism, then $j$ belongs to $\mathcal{L}$;

(3) if $j: U \rightarrow V$ and $k: V \rightarrow X$ belong to $\mathcal{L}$, then $k \circ j$ belongs to $\mathcal{L}$;

(4) if $j: U \rightarrow X$ belongs to $\mathcal{L}$ and $f: X^{\prime} \rightarrow X$ is a morphism in $\mathcal{D}$, then the induced morphism $j^{\prime}: U \times_{X} X^{\prime} \rightarrow X^{\prime}$ belongs to $\mathcal{L}$; and

(5) if $i: U \rightarrow X$ and $j: V \rightarrow X$ belong to $\mathcal{L}$, then there exists a commutative diagram:

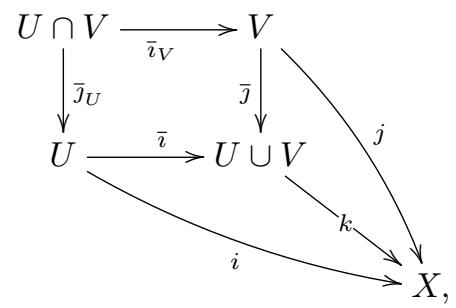

where $k$ belongs to $\mathcal{L}$, and the square is a Mayer-Vietoris $\mathcal{T}$-square. 
Note that $U \cap V=U \times_{X} V$.

Example 5.11. We continue Example 5.6. Let $\mathcal{J}$ be the collection of morphisms in $\boldsymbol{R e p}^{\mathrm{fp}} / Y$ that are open immersions. By Example 5.6 and standard arguments, $\mathcal{J}$ supports $\mathrm{D}_{\mathrm{qc}}$.

We now have a straightforward lemma.

Lemma 5.12. Let $\mathcal{T}$ be a $\mathcal{D}$-presheaf of triangulated categories with adjoints. Let $\mathcal{L}$ be a collection of morphisms in $\mathcal{D}$ that supports $\mathcal{T}$. If $i: U \rightarrow X$ and $j: V \rightarrow X$ belong to $\mathcal{L}$, then

(1) $\mathcal{T}_{X \backslash U \cup V}(X)=\mathcal{T}_{X \backslash U}(X) \cap \mathcal{T}_{X \backslash V}(X)$, and

(2) there is an exact sequence of triangulated categories:

$$
\mathcal{T}_{X \backslash U \cup V}(X) \rightarrow \mathcal{T}_{X \backslash V}(X) \stackrel{i^{*}}{\rightarrow} \mathcal{T}_{U \backslash U \cap V}(U) .
$$

Proof. (1) Certainly, we have $\mathcal{T}_{X \backslash U \cup V}(X) \subseteq\left(\operatorname{ker} i^{*}\right) \cap\left(\operatorname{ker} j^{*}\right)$. For the other inclusion let $M \in\left(\operatorname{ker} i^{*}\right) \cap\left(\operatorname{ker} j^{*}\right)$. If $k: U \cup V \rightarrow X$ denotes the morphism induced by Definition 5.10 5), then $k^{*} M \in \mathcal{T}(U \cup V)$. Now let $\bar{k}: U \cap V \rightarrow U \cup V$ denote the induced morphism. There is a triangle in $\mathcal{T}(U \cup V)$ :

$$
k^{*} M \rightarrow \bar{\imath}_{*} \bar{\imath}^{*} k^{*} M \oplus \bar{\jmath}_{*} \bar{\jmath}^{*} k^{*} M \rightarrow \bar{k}_{*} \bar{k}^{*} k^{*} M .
$$

Functoriality induces isomorphisms $\bar{\imath}^{*} k^{*} \simeq i^{*}, \bar{\jmath}^{*} k^{*} \simeq j^{*}$, and $\bar{k}^{*} k^{*} \simeq \bar{\jmath}_{U}^{*} i^{*}$. By hypothesis $i^{*} M$ and $j^{*} M$ vanish, so the triangle gives $k^{*} M \simeq 0$. Hence $M \in$ $\mathcal{T}_{X \backslash U \cup V}(X)$.

(2) Lemma 5.4 2 shows that the functor $i^{*}: \mathcal{T}_{X \backslash V}(X) \rightarrow \mathcal{T}(U)$ factors through $\mathcal{T}_{U \backslash U \cap V}(U)$ and the kernel is $\left(\operatorname{ker} i^{*}\right) \cap\left(\operatorname{ker} j^{*}\right)=\mathcal{T}_{X \backslash U \cup V}(X)$ by (1). Also, Lemma 5.43 shows that $i_{*}: \mathcal{T}_{U \backslash U \cap V}(U) \rightarrow \mathcal{T}_{X \backslash V}(X)$ is a right adjoint to $i^{*}$ and Lemma 5.4 11 shows that the natural transformation $i^{*} i_{*} \rightarrow$ Id is an isomorphism. It follows that the sequence is exact by Lemma 3.4 .

\section{Descent of COMPACt Generation}

For this section we fix a small category $\mathcal{D}$ that admits all finite limits. We also fix a collection $\mathcal{L}$ of morphisms in $\mathcal{D}$.

Definition 6.1. An admissible $(\mathcal{L}, \mathcal{D})$-presheaf of triangulated categories is a $\mathcal{D}$ presheaf $\mathcal{T}$ of triangulated categories with adjoints (\$5) satisfying

(1) for all $X \in \mathcal{D}$, the triangulated category $\mathcal{T}(X)$ is closed under small coproducts;

(2) for all $(f: X \rightarrow Y) \in \mathcal{D}$, the push-forward $f_{*}: \mathcal{T}(X) \rightarrow \mathcal{T}(Y)$ preserves small coproducts; and

(3) $\mathcal{L}$ supports $\mathcal{T}$ (Definition 5.10.

Example 6.2. We continue Example 5.11. $\mathrm{D}_{\mathrm{qc}}$ is an admissible $\left(\mathcal{J}, \boldsymbol{R e p}^{\mathrm{fp}} / Y\right)$ presheaf of triangulated categories. Indeed, the only non-trivial condition is that $f_{*}$ preserves small coproducts, which follows from Theorem 2.6 3ince representable morphisms are concentrated.

Definition 6.3. Let $\beta$ be a cardinal and let $X \in \mathcal{D}$. We say that an admissible $(\mathcal{L}, \mathcal{D})$-presheaf of triangulated categories $\mathcal{T}$ is compactly generated with $\mathcal{L}$-supports by $\beta$ objects at $X$ if for every $j: V \rightarrow X$ in $\mathcal{L}$ the triangulated category $\mathcal{T}_{X \backslash V}(X)$ is generated by a set of cardinality $\leq \beta$ whose elements have compact image in $\mathcal{T}(X)$.

In practice, $\mathcal{D}$ will often contain an initial object $\emptyset$ and for every $X \in \mathcal{D}$ it will be the case that $(\emptyset \rightarrow X) \in \mathcal{L}$ and $\mathcal{T}(\emptyset) \simeq 0$. Hence, in this situation, $\mathcal{T}(X)=\mathcal{T}_{X \backslash \emptyset}(X)$ is also compactly generated by a set of cardinality $\leq \beta$. Also observe that if $\beta$ is 
a finite cardinal, then $\mathcal{T}$ can always be compactly generated with supports by one object at $X$. In this section we will give conditions on $\mathcal{T}$ that guarantee that the condition of compact generation with $\mathcal{L}$-supports by $\beta$ objects descends along certain morphisms and diagrams in $\mathcal{D}$.

Our first result is of an elementary nature and is similar to the arguments of Toën [Toë12, Lem. 4.11]. First we require a definition:

Definition 6.4. Let $\mathcal{T}$ be an admissible $(\mathcal{L}, \mathcal{D})$-presheaf of triangulated categories. A morphism $f: X^{\prime} \rightarrow X$ in $\mathcal{D}$ is $\mathcal{T}$-quasiperfect with respect to $\mathcal{L}$ if the following three conditions are satisfied:

(1) $f$ is $\mathcal{T}$-flat (Definition 5.1);

(2) if $P \in \mathcal{T}\left(X^{\prime}\right)^{c}$, then $f_{*} P \in \mathcal{T}(X)^{c}$;

(3) $f_{*}$ admits a right adjoint $f^{\times}$such that for every $j: V \rightarrow X$ in $\mathcal{L}$, the restriction of $f^{\times}$to $\mathcal{T}_{X \backslash V}(X)$ factors through $\mathcal{T}_{X^{\prime} \backslash V \times_{X} X^{\prime}}\left(X^{\prime}\right)$.

By Example 3.8, a potentially easy way to verify condition (2) above is for $f^{\times}$to preserve small coproducts. To verify condition (3) above, it is sufficient to prove the following: for every $j: V \rightarrow X$ in $\mathcal{L}$, if $j^{\prime}: V^{\prime} \rightarrow X^{\prime}$ is the pullback of $j$ along $f$ and $f_{V}: V^{\prime} \rightarrow V$ is the projection to $V$, then $f_{*}$ and $\left(f_{V}\right)_{*}$ both admit right adjoints and the natural transformation $j^{\prime *} f^{\times} \rightarrow f_{V}^{\times} j^{*}$ is an isomorphism of functors.

Example 6.5. We continue with Example 6.2. If $q: W^{\prime} \rightarrow W$ is a finite and faithfully flat morphism of finite presentation, then $q$ is $\mathrm{D}_{\mathrm{qc}}$-quasiperfect with respect to J (Corollary 4.15). In [HR15, App. A], we prove that if $q: W^{\prime} \rightarrow W$ is a proper, smooth and locally schematic morphism of noetherian algebraic stacks, then $q$ is $\mathrm{D}_{\mathrm{qc}}$-quasiperfect with respect to $\mathcal{J}$.

We now have the first important result of this section.

Proposition 6.6. Let $\beta$ be a cardinal. Let $\mathcal{T}$ be an admissible $(\mathcal{L}, \mathcal{D})$-presheaf of triangulated categories. Let $f: X^{\prime} \rightarrow X$ be a morphism in $\mathcal{D}$ that is $\mathcal{T}$-quasiperfect with respect to $\mathcal{L}$. If the functor $f^{\times}$(which exists because $f$ is $\mathcal{T}$-quasiperfect) is conservative and $\mathcal{T}$ is compactly generated with $\mathcal{L}$-supports by $\beta$ objects at $X^{\prime}$, then $\mathcal{T}$ is compactly generated with $\mathcal{L}$-supports by $\beta$ objects at $X$. In fact, if $j: V \rightarrow X$ belongs to $\mathcal{L}$, let $V^{\prime}=X^{\prime} \times_{X} V$ and let $\mathcal{B}^{\prime} \subseteq \mathcal{T}\left(X^{\prime}\right)^{c} \cap \mathcal{T}_{X^{\prime} \backslash V^{\prime}}\left(X^{\prime}\right)$ be a subset of cardinality $\leq \beta$ generating $\mathcal{T}_{X^{\prime} \backslash V^{\prime}}\left(X^{\prime}\right)$, then $f_{*} \mathcal{B}^{\prime} \subseteq \mathcal{T}(X)^{c} \cap \mathcal{T}_{X \backslash V}(X)$ and $f_{*} \mathcal{B}^{\prime}$ generates $\mathcal{T}_{X \backslash V}(X)$.

Proof. It suffices to prove the latter assertion. Set $\mathcal{B}=f_{*} \mathcal{B}^{\prime}=\left\{f_{*} P: P \in \mathcal{B}^{\prime}\right\}$. Then $\mathcal{B}$ has cardinality $\leq \beta$, and $\mathcal{B} \subseteq \mathcal{T}_{X \backslash V}(X)$ by Lemma 5.4 3. Moreover, $\mathcal{B} \subseteq \mathcal{T}(X)^{c}$, since $f$ is $\mathcal{T}$-quasiperfect with respect to $\mathcal{L}$. It remains to show that $\mathcal{B}$ generates $\mathcal{T}_{X \backslash V}(X)$. Let $N \in \mathcal{T}_{X \backslash V}(X)$ satisfy $\operatorname{Hom}_{\mathcal{T}(X)}\left(f_{*} P[n], N\right)=0$ for all $P \in \mathcal{B}^{\prime}$ and all $n \in \mathbb{Z}$. Since $f$ is $\mathcal{T}$-quasiperfect with respect to $\mathcal{L}$, it follows that $f^{\times} N \in \mathcal{T}_{X^{\prime} \backslash V^{\prime}}\left(X^{\prime}\right)$. As $\mathcal{B}^{\prime}$ is generating for $\mathcal{T}_{X^{\prime} \backslash V^{\prime}}\left(X^{\prime}\right)$, we may conclude that $f^{\times} N \simeq 0$. By assumption, $f^{\times}$is conservative. Thus $N \simeq 0$ and $\mathcal{B}$ generates $\mathcal{T}_{X \backslash V}(X)$.

Our next descent result is deeper, relying on Thomason's Localization Theorem 3.12. First, however, we require a lemma.

Lemma 6.7. Let $\beta$ be a cardinal. Let $\mathcal{T}$ be an admissible $(\mathcal{L}, \mathcal{D})$-presheaf of triangulated categories. Suppose that $\mathcal{T}$ is compactly generated with $\mathcal{L}$-supports by $\beta$ objects at $X \in \mathcal{D}$. Let $W \rightarrow V$ and $V \rightarrow X$ belong to $\mathcal{L}$. Then the following holds.

(1) $\mathcal{T}_{X \backslash W}(X)$ is closed under small coproducts and the subcategory $\mathcal{T}_{X \backslash V}(X) \subseteq$ $\mathcal{T}_{X \backslash W}(X)$ is localizing;

(2) $\mathcal{T}_{X \backslash V}(X)$ is the localizing envelope of a set of compact objects of $\mathcal{T}_{X \backslash W}(X)$; and 
(3) $\mathcal{T}_{X \backslash V}(X)^{c}=\mathcal{T}_{X \backslash W}(X)^{c} \cap \mathcal{T}_{X \backslash V}(X)$.

Proof. First, observe that $\mathcal{T}(X)$ is closed under small coproducts. Also, if $f: X^{\prime} \rightarrow$ $X$ is a morphism in $\mathcal{D}$, then $f^{*}$ admits a right adjoint, so $f^{*}$ preserves small coproducts. Hence, we see that $\mathcal{T}_{X \backslash X^{\prime}}(X)$ is a localizing subcategory of $\mathcal{T}(X)$. In particular, $\mathcal{T}_{X \backslash X^{\prime}}(X)$ is closed under small coproducts. The claim (1) is now immediate.

By hypothesis, $\mathcal{T}_{X \backslash V}(X)$ is generated by a subset $R$ that has compact image in $\mathcal{T}(X)$, hence also in $\mathcal{T}_{X \backslash W}(X)$. Let $\mathcal{R} \subseteq \mathcal{T}_{X \backslash W}(X)$ denote the localizing envelope of $R$, then $\mathcal{R} \subseteq \mathcal{T}_{X \backslash V}(X)$. Applying Corollary 3.14 to $R \subseteq \mathcal{T}_{X \backslash V}(X)$, we find $\mathcal{R}=\mathcal{T}_{X \backslash V}(X)$, proving (2). The claim (3) is now an immediate consequence of (2) and Thomason's Theorem 3.12 .

Proposition 6.8. Let $\beta$ be a cardinal. Let $\mathcal{T}$ be an admissible $(\mathcal{L}, \mathcal{D})$-presheaf of triangulated categories. Consider a Mayer-Vietoris $\mathcal{T}$-square (Definition 5.5):

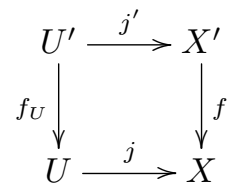

with $j \in \mathcal{L}$. If $\mathcal{T}$ is compactly generated with $\mathcal{L}$-supports by $\beta$ objects at $U$ and $X^{\prime}$, then $\mathcal{T}$ is compactly generated with $\mathcal{L}$-supports by $\beta$ objects at $X$.

Proof. Let $V \rightarrow X$ belong to $\mathcal{L}$. Form the cartesian cube:

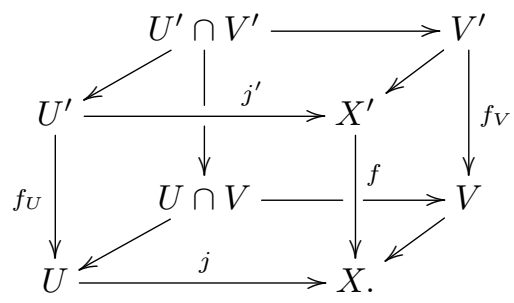

By Lemma 5.12, we have an exact sequence

$$
\mathcal{T}_{X^{\prime} \backslash U^{\prime} \cup V^{\prime}}\left(X^{\prime}\right) \rightarrow \mathcal{T}_{X^{\prime} \backslash V^{\prime}}\left(X^{\prime}\right) \rightarrow \mathcal{T}_{U^{\prime} \backslash U^{\prime} \cap V^{\prime}}\left(U^{\prime}\right) .
$$

The category $\mathcal{T}_{X^{\prime} \backslash V^{\prime}}\left(X^{\prime}\right)$ is compactly generated and by Lemma 6.711) it is also closed under small coproducts. By Lemma 6.72 the subcategory $\mathcal{T}_{X^{\prime} \backslash U^{\prime} \cup V^{\prime}}\left(X^{\prime}\right) \subseteq$ $\mathcal{T}_{X^{\prime} \backslash V^{\prime}}\left(X^{\prime}\right)$ is the localizing envelope of a set of compact objects of $\mathcal{T}_{X^{\prime} \backslash V^{\prime}}\left(X^{\prime}\right)$.

Now let $P \in \mathcal{T}(U)^{c} \cap \mathcal{T}_{U \backslash U \cap V}(U)$. Then $f_{U}^{*} P \in \mathcal{T}\left(U^{\prime}\right)^{c}$ since $\left(f_{U}\right)_{*}$ preserves coproducts (Example 3.8) and $f_{U}^{*} P \in \mathcal{T}_{U^{\prime} \backslash U^{\prime} \cap V^{\prime}}\left(U^{\prime}\right)$ (Lemma 5.4 2). Thus $f_{U}^{*} P \in$ $\mathcal{T}_{U^{\prime} \backslash U^{\prime} \cap V^{\prime}}\left(U^{\prime}\right)^{c}$ by Lemma 6.7 3). We now apply Thomason's localization Theorem, in the form of Corollary 3.13. to the exact sequence 6.1). This gives us $P^{\prime} \in$ $\mathcal{T}_{X^{\prime} \backslash V^{\prime}}\left(X^{\prime}\right)^{c}=\mathcal{T}\left(X^{\prime}\right)^{c} \cap \mathcal{T}_{X^{\prime} \backslash V^{\prime}}\left(X^{\prime}\right)$ and an isomorphism $j^{\prime *} P^{\prime} \simeq f_{U}^{*}(P \oplus P[1])$. As in Lemma 5.93, form the following triangle in $\mathcal{T}(X)$ :

$$
\tilde{P} \rightarrow j_{*}(P \oplus P[1]) \oplus f_{*} P^{\prime} \rightarrow f_{*} f^{*} j_{*}(P \oplus P[1]) .
$$

By Lemma 5.9 3 4 we have that $j^{*} \tilde{P} \simeq P \oplus P[1]$ and $f^{*} \tilde{P} \simeq P^{\prime}$ and that $\tilde{P} \in \mathcal{T}(X)^{c}$. Since $j_{*} j^{*} \tilde{P}, f_{*} f^{*} \tilde{P}$ and $f_{*} f^{*} j_{*} j^{*} \tilde{P} \in \mathcal{T}_{X \backslash V}(X)$, it follows that $\tilde{P} \in \mathcal{T}_{X \backslash V}(X)$.

Now let $Q \in \mathcal{T}_{X^{\prime} \backslash U^{\prime} \cup V^{\prime}}\left(X^{\prime}\right)$, and note that $f_{*} Q \in \mathcal{T}_{X \backslash U \cup V}(X)$ (Lemma 5.12 (1)). Moreover, $f^{*} f_{*} Q \rightarrow Q$ is an isomorphism, because $f^{*}: \mathcal{T}_{X \backslash U}(X) \rightarrow \mathcal{T}_{X^{\prime} \backslash U^{\prime}}\left(X^{\prime}\right)$ is an equivalence of categories. We also have that $j^{*} f_{*} Q \simeq 0$ and $j^{*} f^{*} f_{*} Q \simeq 0$. Thus, if in addition $Q \in \mathcal{T}\left(X^{\prime}\right)^{c}$, then $f_{*} Q \in \mathcal{T}(X)^{c}$ by Lemma 5.94. 
By hypothesis, there is a subset $\mathcal{B}_{0} \subseteq \mathcal{T}(U)^{c} \cap \mathcal{T}_{U \backslash U \cap V}(U)$ (resp. $\mathcal{B}_{1} \subseteq \mathcal{T}\left(X^{\prime}\right)^{c} \cap$ $\left.\mathcal{T}_{X^{\prime} \backslash U^{\prime} \cup V^{\prime}}\left(X^{\prime}\right)\right)$ of cardinality $\leq \beta$ generating $\mathcal{T}_{U \backslash U \cap V}(U)\left(\operatorname{resp} . \mathcal{T}_{X^{\prime} \backslash U^{\prime} \cup V^{\prime}}\left(X^{\prime}\right)\right)$. Define:

$$
\mathcal{B}=\left\{\tilde{P}: P \in \mathcal{B}_{0}\right\} \cup\left\{f_{*} Q: Q \in \mathcal{B}_{1}\right\} .
$$

If $\beta$ is infinite, then the cardinality of $\mathcal{B}$ is $\leq \beta$, and if $\beta$ is finite then the same is true of $\mathcal{B}$. By the above considerations, $\mathcal{B} \subseteq \mathcal{T}(X)^{c} \cap \mathcal{T}_{X \backslash V}(X)$ and it remains to show that $\mathcal{B}$ generates $\mathcal{T}_{X \backslash V}(X)$.

Let $M \in \mathcal{T}_{X \backslash V}(X)$ so that $f^{*} M \in \mathcal{T}_{X^{\prime} \backslash V^{\prime}}\left(X^{\prime}\right)$ and $j^{*} M \in \mathcal{T}_{U \backslash U \cap V}(U)$. Suppose that $\operatorname{Hom}_{\mathcal{T}(X)}\left(f_{*} Q[n], M\right)=0$ for all $Q \in \mathcal{B}_{1}$ and all $n \in \mathbb{Z}$. By Lemma 5.9 2), we see that $\operatorname{Hom}_{\mathcal{T}\left(X^{\prime}\right)}\left(Q[n], f^{*} M\right)=0$ for all $Q \in \mathcal{B}_{1}$ and all $n \in \mathbb{Z}$. Let $K$ be a cone of $f^{*} M \rightarrow j_{*}^{\prime} j^{\prime *} f^{*} M$. Note that

$$
\operatorname{Hom}_{\mathcal{T}\left(X^{\prime}\right)}\left(Q[n], j_{*}^{\prime} j^{\prime *} f^{*} M\right)=\operatorname{Hom}_{\mathcal{T}\left(X^{\prime}\right)}\left(j^{\prime *} Q[n], j^{\prime *} f^{*} M\right)=0
$$

so $\operatorname{Hom}_{\mathcal{T}\left(X^{\prime}\right)}(Q[n], K)=0$. Since $K \in \mathcal{T}_{X^{\prime} \backslash U^{\prime} \cup V^{\prime}}\left(X^{\prime}\right)$ and $\mathcal{B}_{1}$ is generating, we see that $K \simeq 0$, so $f_{*} f^{*} M \rightarrow f_{*} j_{*}^{\prime} j^{* *} f^{*} M \simeq f_{*} f^{*} j_{*} j^{*} M$ is an isomorphism. From the Mayer-Vietoris triangle $M \rightarrow j_{*} j^{*} M \oplus f_{*} f^{*} M \rightarrow f_{*} f^{*} j_{*} j^{*} M$, we deduce that the natural map $M \rightarrow j_{*} j^{*} M$ is an isomorphism for all such $M$.

Now suppose that $M$ also satisfies $\operatorname{Hom}_{\mathcal{T}(X)}(\tilde{P}[n], M)=0$ for all $P \in \mathcal{B}_{0}$ and $n \in \mathbb{Z}$. Since the natural map $M \rightarrow j_{*} j^{*} M$ is an isomorphism, it follows that $\operatorname{Hom}_{\mathcal{T}(U)}\left(j^{*} \tilde{P}[n], j^{*} M\right)=0$ for all $P \in \mathcal{B}_{0}$ and $n \in \mathbb{Z}$. By Lemma 5.9 (3), $j^{*} \tilde{P} \simeq$ $P \oplus P[1]$ and so $\operatorname{Hom}_{\mathcal{T}(U)}\left(P[n], j^{*} M\right)=0$ for all $P \in \mathcal{B}_{0}$ and all $n \in \mathbb{Z}$. By assumption, $\mathcal{B}_{0}$ generates $\mathcal{T}_{U \backslash U \cap V}(U)$ and thus $j^{*} M \simeq 0$. Since $M \simeq j_{*} j^{*} M \simeq 0$, we deduce that $\mathcal{B}$ generates $\mathcal{T}_{X \backslash V}(X)$.

We are now in a position to prove the main technical result of the article.

Theorem 6.9. Let $X$ be a quasi-compact and quasi-separated algebraic stack. Let $\mathcal{D}$ be $\boldsymbol{R e p}^{\mathrm{fp}} / X$ or one of the full subcategories $\boldsymbol{R e p}^{\mathrm{fp}, \mathrm{qff}, \mathrm{sep}} / X$ or $\boldsymbol{R e p}^{\mathrm{fp}, \mathrm{e} t, \mathrm{sep}} / X$. Let $\mathcal{J}$ denote the set of open immersions in $\mathcal{D}$. Let $\mathcal{T}$ be a presheaf of triangulated categories on $\mathcal{D}$. Assume that

(1) $\mathcal{T}(W)$ is closed under small coproducts for all $W \in \mathcal{D}$,

(2) for every morphism $f: W_{1} \rightarrow W_{2}$ in $\mathcal{D}$, the pullback $f^{*}: \mathcal{T}\left(W_{2}\right) \rightarrow \mathcal{T}\left(W_{1}\right)$ admits a right adjoint $f_{*}$ that preserves small coproducts,

(3) for every cartesian square in $\mathcal{D}$

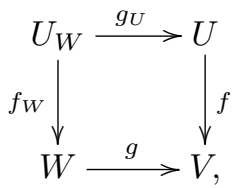

such that $g$ is flat, the natural transformation $g^{*} f_{*} \rightarrow\left(f_{W}\right)_{*}\left(g_{U}\right)^{*}$ is an isomorphism,

(4) for every open immersion $U \rightarrow W$ and étale neighborhood $f: W^{\prime} \rightarrow W$ of $W \backslash U$, the pullback $f^{*}$ induces an equivalence $\mathcal{T}_{W \backslash U}(W) \rightarrow \mathcal{T}_{W^{\prime} \backslash U^{\prime}}\left(W^{\prime}\right)$,

(5) for every finite faithfully flat morphism $W^{\prime} \rightarrow W$ of finite presentation, the functor $f_{*}: \mathcal{T}\left(W^{\prime}\right) \rightarrow \mathcal{T}(W)$ admits a right adjoint $f^{\times}$that preserves small coproducts, is conservative, and commutes with pullback along open immersions.

Let $\mathcal{C} \subseteq \mathcal{D}$ be the collection of all objects $W$ such that for every separated étale morphism $q: W^{\prime} \rightarrow W$ in $\mathcal{D}$ and every open immersion $V^{\prime} \rightarrow W^{\prime}$ in $\mathcal{J}$, the triangulated category $\mathcal{T}_{W^{\prime} \backslash V^{\prime}}\left(W^{\prime}\right)$ is generated by a set of cardinality $\leq \beta$ whose elements have compact image in $\mathcal{T}\left(W^{\prime}\right)$.

If $p: W \rightarrow X$ is a separated, quasi-finite and faithfully flat morphism in $\mathcal{D}$ such that $W \in \mathcal{C}$, then $X \in \mathcal{C}$. 
Proof. Condition (2) says that $\mathcal{T}$ has adjoints. Condition (3) says that flat morphisms are $\mathcal{T}$-flat. Conditions (3) and (4) imply that étale neighborhoods are Mayer-Vietoris squares. In particular, $\mathcal{J}$ supports $\mathcal{T}$. Combining this with conditions (1) and (2), we conclude that $\mathcal{T}$ is an admissible $(\mathcal{J}, \mathcal{D})$-presheaf of triangulated categories.

By assumption, there exists an object $W \in \mathcal{C}$ with $W \rightarrow X$ separated, quasifinite and faithfully flat. We will apply Ryd11, Thm. 6.1] to deduce that $X \in \mathcal{C}$. To do this, we need to verify the following three conditions for a flat morphism $q: W^{\prime} \rightarrow W$ in $\mathcal{D}$.

(D1) If $W \in \mathcal{C}$ and $q$ is étale and separated, then $W^{\prime} \in \mathcal{C}$;

(D2) if $W^{\prime} \in \mathcal{C}$ and $q$ is finite and surjective, then $W \in \mathcal{C}$; and

(D3) if $q$ is an étale neighborhood of $W \backslash U$, where $U \rightarrow W$ is an open immersion in $\mathcal{D}$, and $U$ and $W^{\prime}$ belong to $\mathcal{C}$, then $W \in \mathcal{C}$.

Now (D1) tautologically follows from the definition of $\mathcal{C}$. For (D2), Condition (5) implies that $p$ is $\mathcal{T}$-quasiperfect with respect to $\mathcal{J}$. By Proposition 6.6, we deduce that (D2) is satisfied. As noted previously, Conditions (3) and (4) imply that étale neighborhoods are Mayer-Vietoris $\mathcal{T}$-squares. By Proposition 6.8. (D3) is satisfied. The result follows.

\section{Algebraic stacks with the $\beta$-Resolution Property}

Let $X$ be an algebraic stack. Recall that $X$ is said to have the resolution property if every quasi-coherent $\mathcal{O}_{X}$-module $M$ is a quotient of a direct sum of locally free $\mathcal{O}_{X^{-}}$ modules of finite type. The resolution property is a subtle and difficult property, although it is always satisfied for quasi-projective schemes. It has been studied systematically by several authors, with notable contributions due to Thomason Tho87, Totaro [Tot04, and Gross Gro10, Gro17.

The following simple refinements of the resolution property will be useful for us. Let $\mathbf{V B}(X) \subseteq \mathrm{Q} \operatorname{Coh}(X)$ denote the subcategory of locally free $\mathcal{O}_{X}$-modules of finite type. Let $\beta$ be a cardinal. We say that $X$ has the $\beta$-resolution property if there exists a subset $\mathcal{B} \subseteq \mathbf{V B}(X)$ of cardinality $\leq \beta$, with the property that every quasi-coherent $\mathcal{O}_{X}$-module $M$ is a quotient of a direct sum of elements of $\mathcal{B}$. If, in addition, it can be arranged that $\mathcal{B}$ consists of vector bundles that are compact objects of $\mathrm{D}_{\mathrm{qc}}(X)$, then we say that $X$ has the compact $\beta$-resolution property.

If $X$ is concentrated, then every locally free $\mathcal{O}_{X}$-module of finite type is a compact object of $\mathrm{D}_{\mathrm{qc}}(X)$ (Lemma 4.4 3) ). In particular, if $X$ also has the $\beta$-resolution property, then $X$ has the compact $\beta$-resolution property. Since quasi-compact, quasi-separated schemes and algebraic spaces are concentrated, the compact $\beta$ resolution property and the $\beta$-resolution property coincide for schemes and algebraic spaces.

The following simple Lemma will be important.

Lemma 7.1 (Gro17, Prop. $1.8(\mathrm{v})]$ ). Let $f: X \rightarrow Y$ be a quasi-affine morphism of algebraic stacks. Let $\beta$ be a cardinal. If $Y$ has the $\beta$-resolution property or the compact $\beta$-resolution property, then so does $X$.

Proof. If $\mathcal{B} \subseteq \mathbf{V B}(Y)$ is a resolving set of cardinality $\beta$, then $f^{*} \mathcal{B}=\left\{f^{*} E: E \in\right.$ $\mathcal{B}\}$ is a resolving set of cardinality $\beta$. Indeed, $f^{*}$ is right-exact and $f^{*} f_{*} M \rightarrow M$ is surjective for every $M \in \mathrm{Q} \operatorname{Coh}(X)$. In addition, if $\mathcal{B}$ consists of compact objects of $\mathrm{D}_{\mathrm{qc}}(Y)$, then $f^{*} \mathcal{B}$ consists of compact objects in $\mathrm{D}_{\mathrm{qc}}(X)$ (Example 3.9

Remark 7.2. Similarly, there is the following partial converse: if $f: X \rightarrow Y$ is finite and faithfully flat of finite presentation and $X$ has the $\beta$-resolution property or compact $\beta$-resolution property, then so does $Y$. In this case, one takes $f_{*} \mathcal{B}$ as 
the resolving set and uses that $f_{*}$ is right-exact and that $f_{*} f^{!} M \rightarrow M$ is surjective [Gro17, Prop. 1.13]. But $f^{!}=f^{\times}$preserves coproducts (Corollary 4.15), so $f_{*}$ preserves compact objects (Example 3.8.

Proposition 7.3. Let $X$ be an algebraic stack. If $X$ is quasi-affine, then $X$ has the 1-resolution property. If $X$ is quasi-compact and quasi-separated with affine stabilizer groups, then the following are equivalent:

(1) $X$ has the $\aleph_{0}$-resolution property;

(2) $X$ has the resolution property; and

(3) $X=\left[V / \mathrm{GL}_{n, \mathbb{Z}}\right]$ where $V$ is a quasi-affine scheme.

When these conditions hold, $X$ has affine diagonal.

Proof. The first statement follows from the fact that $\mathcal{O}_{X} \in \mathrm{Q} \operatorname{Coh}(X)$ is a generator if $X$ is quasi-affine. Trivially, $\sqrt{1} \Longrightarrow(2)$. That $\sqrt{2} \Longrightarrow(3)$ is Totaro's theorem Tot04, Gro17. Finally, to see that $(3) \Longrightarrow(1)$ it is enough to prove that $B \mathrm{GL}_{n, \mathbb{Z}}$ has the $\aleph_{0}$-resolution property since $X \rightarrow B \mathrm{GL}_{n, \mathbb{Z}}$ is quasi-affine. That $B \mathrm{GL}_{n, \mathbb{Z}}$ has the resolution property is a special case of [Tho87, Lem. 2.4]: every coherent sheaf on $B G L_{n, \mathbb{Z}}$ is a quotient of a finite-dimensional subrepresentation of a finite number of copies of the regular representation. Since there is a countable number of vector bundles on $B G L_{n, \mathbb{Z}}$, the $\aleph_{0}$-resolution property holds.

The last statement follows since $\left[V / \mathrm{GL}_{n, \mathbb{Z}}\right]$ has affine diagonal.

Question 7.4. Every algebraic stack that admits a finite flat cover $V \rightarrow X$ with $V$ quasi-affine has the compact 1-resolution property by Remark 7.2. Are all stacks with the (compact) 1-resolution property of this form? Is every algebraic space with the 1-resolution property quasi-affine?

Many quotient stacks have the resolution property:

Example 7.5. Let $S=\operatorname{Spec} R$ be a regular scheme of dimension at most 1 (e.g., $R=\mathbb{Z}$ or $R$ is a field). Let $G \rightarrow S$ be a flat affine group scheme of finite type and let $V$ be an algebraic space with an action of $G$. Then $X=[V / G]$ has the resolution property in the following cases [Tot04, Thm. 2.1]:

(1) $V$ is quasi-affine.

(2) $V$ is normal, noetherian and has an ample family of line bundles (e.g. $V$ quasi-projective) and $G$ is an extension of a finite flat group scheme by a smooth group scheme with connected fibers (this is automatic if $R$ is a field).

(3) $V$ has an ample family of $G$-equivariant line bundles (e.g. $V$ is quasiprojective and $G$ is acting linearly).

For (1), use that $B G_{S}$ has the resolution property Tho87, Lem. 2.4] and Lemma 7.1. For (2) use [Tho87, Lem. 2.10 and 2.14] and for (3) use [Tho87, Lem. 2.6]. Note that in (2) it is crucial that $V$ is normal to apply Sumihiro's theorem [Sum75, Thm. 1.6] and deduce that sufficiently high powers of the line bundles carry a $G$-action. In fact, this is false otherwise (cf. [Tot04, §9]) and whether $X$ has the resolution property in this case is not known in general. Alternatively, we could assume that $V$ is a quasi-projective scheme with a linear action of $G$, as in BZFN10, Cor. 3.22].

We conclude this section with a simple lemma.

Lemma 7.6. Let $X$ be a quasi-compact and quasi-separated algebraic stack. The following conditions are equivalent:

(1) $X$ has the compact $\beta$-resolution property.

(2) $X$ has the $\beta$-resolution property and there exists a vector bundle $F$ such that $\operatorname{supp}(F)=X$ and $F$ is a compact object of $\mathrm{D}_{\mathrm{qc}}(X)$. 
(3) There is a vector bundle $F$ on $X$ that is a compact object of $\mathrm{D}_{\mathrm{qc}}(X)$ and a subset $\mathcal{B} \subseteq \mathbf{V B}(X)$ of cardinality $\leq \beta$ such that the set $\{F \otimes E\}_{E \in \mathcal{B}}$ generates $\mathrm{QCoh}(X)$.

(4) There is a subset $\mathcal{B} \subseteq \mathbf{V B}(X)$ of cardinality $\leq \beta$ that generates $\mathrm{Q} \operatorname{Coh}(X)$ and an integer $r \geq 0$ such that $\operatorname{Ext}_{\mathcal{O}_{X}}^{i}(E, N)=0$ for all $i>r$, all $E \in \mathcal{B}$ and all $N \in \mathrm{Q} \operatorname{Coh}(X)$.

(5) There is a subset $\mathcal{B} \subseteq \mathbf{V B}(X)$ of cardinality $\leq \beta$ that generates $\mathrm{Q} \operatorname{Coh}(X)$ and an integer $r \geq 0$ such that the natural map

$$
\tau^{\geq j} \operatorname{RHom}_{\mathcal{O}_{X}}(E, \mathcal{M}) \rightarrow \tau^{\geq j} \operatorname{RHom}_{\mathcal{O}_{X}}\left(E, \tau^{\geq j-r} \mathcal{M}\right)
$$

is a quasi-isomorphism for all integers $j$, all $E \in \mathcal{B}$ and all $\mathcal{M} \in \mathrm{D}_{\mathrm{qc}}(X)$.

Proof. $(1) \Rightarrow(2)$ : there is a surjection $F \rightarrow O_{X}$ with $F$ a vector bundle on $X$ that is compact in $\mathrm{D}_{\mathrm{qc}}(X)$. Note that $\operatorname{supp}(F)=X$.

$(2) \Rightarrow(3)$ : If $\mathcal{C} \subseteq \mathbf{V B}(X)$ is a resolving set of vector bundles, then so is the set $\left\{F \otimes F^{\vee} \otimes E\right\}_{E \in \mathcal{C}}$. Indeed, the evaluation map $F \otimes F^{\vee} \rightarrow \mathcal{O}_{X}$ is surjective. We take $\mathcal{B}=\left\{F^{\vee} \otimes E\right\}_{E \in \mathcal{C}}$.

$(3) \Rightarrow(4):$ for all $i$, all $E \in \mathcal{B}$, and $N \in \mathrm{Q} \operatorname{Coh}(X)$ we have $\operatorname{Ext}_{\mathcal{O}_{X}}^{i}(F \otimes E, N)=$ $\operatorname{Ext}_{\mathcal{O}_{X}}^{i}\left(F, E^{\vee} \otimes N\right)$. Now choose $r \geq 0$ as in Lemma 4.5 for $F$. An identical argument gives $(3) \Rightarrow(5)$. Also, $(5) \Rightarrow(4)$ is trivial.

(4) $\Rightarrow$ (1): immediate from Lemma 4.5 .

\section{CRISP STACKS}

In this section we define $\beta$-crisp stacks and show that the compact $\beta$-resolution property implies $\beta$-crispness.

Definition 8.1. Let $\beta$ be a cardinal. Let $X$ be an algebraic stack. We say that $X$ satisfies the $\beta$-Thomason condition if:

(1) $\mathrm{D}_{\mathrm{qc}}(X)$ is compactly generated by a set of cardinality $\leq \beta$; and

(2) for every quasi-compact open immersion $j: U \hookrightarrow X$ there exists a perfect object $P$ of $\mathrm{D}_{\mathrm{qc}}(X)$ with support $X \backslash U$.

We say that $X$ is $\beta$-crisp if for every representable, étale, separated, and quasicompact morphism $X^{\prime} \rightarrow X$ the stack $X^{\prime}$ satisfies the $\beta$-Thomason condition.

By Lemmas 4.10 and 8.2 , an equivalent definition for $\beta$-crispness is that for every representable étale morphism $W \rightarrow X$ that is quasi-compact and separated, and every quasi-compact open immersion $j: U \hookrightarrow W$, the triangulated category

$$
\mathrm{D}_{\mathrm{qc},|W \backslash \backslash U|}(W)=\left\{M \in \mathrm{D}_{\mathrm{qc}}(W): j^{*} M \simeq 0\right\}
$$

is generated by a set of cardinality $\leq \beta$ consisting of compact objects of $\mathrm{D}_{\mathrm{qc}}(W)$.

Lemma 8.2. Let $f: X \rightarrow Y$ be a quasi-affine morphism of algebraic stacks. Let $\beta$ be a cardinal. If $\mathrm{D}_{\mathrm{qc}}(Y)$ is compactly generated by $\beta$ objects, then so is $\mathrm{D}_{\mathrm{qc}}(X)$. In fact, if $\mathcal{B} \subseteq \mathrm{D}_{\mathrm{qc}}(Y)^{c}$ is a subset that generates $\mathrm{D}_{\mathrm{qc}}(Y)$, then the set $\left\{\mathrm{L}_{\mathrm{qc}}^{*} B: B \in\right.$ $B$ \} compactly generates $\mathrm{D}_{\mathrm{qc}}(X)$.

Proof. Since $f$ is concentrated, $\mathrm{R}\left(f_{\mathrm{qc}}\right)_{*}$ preserves small coproducts. Since $\mathrm{R}\left(f_{\mathrm{qc}}\right)_{*}$ is conservative (Corollary 2.8), the result follows from Example 3.11 .

Remark 8.3. We do not know if the analogue of Lemma 8.2 holds for the $\beta$ Thomason condition or even $\beta$-crispness.

The main result of this section is the following 
Proposition 8.4. Let $X$ be a quasi-compact algebraic stack with affine diagonal. Let $\beta$ be a cardinal. If $X$ has the compact $\beta$-resolution property, then it is $\beta$-crisp. In particular, concentrated stacks with the $\beta$-resolution property are $\beta$-crisp. In fact, $\mathrm{D}_{\mathrm{qc}}(X)$ is compactly generated by any resolving set of compact vector bundles.

Proof. By Lemma 7.1 the compact $\beta$-resolution property is preserved under quasiaffine morphisms. By Zariski's Main Theorem [LMB, Thm. 16.5] étale morphisms that are quasi-compact, separated, and representable are quasi-affine. Thus it is enough to prove the following statement: if $j: V \hookrightarrow X$ is a quasi-compact open immersion with complement $|Z|$, then there exists a generating subset $\mathcal{B}_{|Z|} \subseteq$ $\mathrm{D}_{\mathrm{qc},|Z|}(X)$, of cardinality $\leq \beta$, with compact image in $\mathrm{D}_{\mathrm{qc}}(X)$.

Choose a resolving set $\mathcal{B} \subseteq \mathbf{V B}(X)$ of cardinality $\leq \beta$ and an integer $r \geq 0$ as in Lemma 7.6 Let $M \in \mathrm{D}_{\mathrm{qc}}(X)$. We claim that if $n \in \mathbb{Z}$ is such that $\mathcal{H}^{n}(M) \neq 0$, then there exists an $E \in \mathcal{B}$ and a non-zero morphism $E[-n] \rightarrow M$ in $\mathrm{D}_{\mathrm{qc}}(X)$. We prove this claim by a small modification (which is likely well-known-e.g. DG13, Rem. 1.2.10]) to the argument of A. Neeman [Nee96, Ex. 1.10].

Thus, for all $E \in \mathcal{B}$, all $n \in \mathbb{Z}$ and all $M \in \mathrm{D}_{\mathrm{qc}}(X)$ we have

$$
\operatorname{Hom}_{\mathcal{O}_{X}}(E, M[n])=\operatorname{Hom}_{\mathcal{O}_{X}}\left(E, \tau^{\geq-r} M[n]\right) \text {. }
$$

We may consequently assume that $M \in \mathrm{D}_{\mathrm{qc}}^{+}(X)$. By [ur04, Thm. 3.8], the natural functor $\mathrm{D}^{+}(\mathrm{Q} \operatorname{Coh}(X)) \rightarrow \mathrm{D}_{\mathrm{qc}}^{+}(X)$ is an equivalence of triangulated categories.

Hence, we are free to assume that $M$ is a complex $\left(\cdots \rightarrow M^{k} \stackrel{d^{k}}{\rightarrow} M^{k+1} \rightarrow \cdots\right)$ of quasi-coherent $\mathcal{O}_{X}$-modules. By assumption $\mathcal{H}^{n}(M) \neq 0$, so there exists $E \in \mathcal{B}$ and a morphism $E \rightarrow \operatorname{ker}\left(d^{n}\right)$ such that $E \rightarrow \operatorname{ker}\left(d^{n}\right) \rightarrow \mathcal{H}^{n}(M)$ is non-zero. The composition $E \rightarrow \operatorname{ker}\left(d^{n}\right) \rightarrow M^{n}$ thus induces a non-zero morphism $E \rightarrow M[n]$ in $\mathrm{D}_{\mathrm{qc}}(X)$ and we deduce the claim.

We now return to the proof of the Proposition. The above considerations shows that the set $\mathcal{B}$ compactly generates $\mathrm{D}_{\mathrm{qc}}(X)$. Now let $i: Z \hookrightarrow X$ be a closed immersion with support $|Z|$. Since $X$ has the resolution property and $j: V \rightarrow X$ is quasi-compact, we may choose $i$ such that the quasi-coherent ideal sheaf $I$ defining $Z$ in $X$ is of finite type. It follows that there is a surjection $F \rightarrow I$, where $F$ is a finite direct sum of objects of $\mathcal{B}$. Corresponding to the morphism $s: F \rightarrow \mathcal{O}_{X}$, we obtain a section $s^{\vee} \in \Gamma\left(X, F^{\vee}\right)$ with vanishing locus $|Z|$. If $K\left(s^{\vee}\right)$ is the resulting Koszul complex [FL85, IV.2], then $K\left(s^{\vee}\right)$ is a perfect complex on $X$ with support $|Z|$. By Lemma 4.10 2, we deduce the claim.

We conclude this section with examples of algebraic stacks that are crisp.

Example 8.5. Let $A$ be a ring. Then $\operatorname{Spec} A$ is 1 -crisp. Indeed, $\operatorname{Spec} A$ has the 1-resolution property and is concentrated, thus the result follows from Proposition 8.4

Example 8.6. Let $X$ be a concentrated stack with affine stabilizers and the resolution property. Then $X$ has the $\aleph_{0}$-resolution property and affine diagonal (Proposition 7.3), hence is $\aleph_{0}$-crisp (Proposition 8.4).

Examples are stacks of the form $X=[V / G]$ where $V$ and $G$ are as in Example 7.5 and either $S=\operatorname{Spec}(\mathbb{Q})$ or $G$ is linearly reductive (e.g., a torus). Indeed, under these assumptions on $G$, the classifying stack $B G$ is concentrated, so $X$ is concentrated since $X \rightarrow B G$ is representable. More generally, we can take any stack $X=[V / G]$ as in Example 7.5 with linearly reductive stabilizers. Such stacks are concentrated by [HR15, Thm. C].

Remark 8.7. Let $X$ be a quasi-compact algebraic stack with affine diagonal and the resolution property. When $X$ is concentrated, then $\mathrm{D}_{\mathrm{qc}}(X)$ is compactly generated (Proposition 8.4) and $\mathrm{D}_{\mathrm{qc}}(X)$ is an example of a unital algebraic stable homotopy 
category [HPS97, Def. 1.1.4]. Note that the localizing envelope of a set of compact generators is the whole category (Corollary 3.14).

The proof of Proposition 8.4 actually shows that even if $X$ is not concentrated, then $\mathrm{D}(\mathrm{Q} \operatorname{Coh}(X))$ is perfectly generated. Note that since $\mathrm{D}(\mathrm{Q} \operatorname{Coh}(X))$ may not be compactly generated, Corollary 3.14 does not apply. Nonetheless $\mathrm{D}(\mathrm{QCoh}(X))$ is well-generated [Nee01a, Thm. 0.2] and there is a version of Corollary 3.14 for wellgenerated triangulated categories [Nee01b, Thm. 1.14]. This result and others are also discussed in [AJPV17]. Thus, $\mathrm{D}(\mathrm{Q} \operatorname{Coh}(X))$ is a non-algebraic stable homotopy category in the sense of [HPS97, Def. 1.1.4]. Note that this says nothing about perfect or compact generation of $\mathrm{D}_{\mathrm{qc}}(X)$, because the functor $\mathrm{D}(\mathrm{Q} \operatorname{Coh}(X)) \rightarrow$ $\mathrm{D}_{\mathrm{qc}}(X)$ can fail to be fully faithful or essentially surjective (e.g., if $X=B \mathbb{G}_{a}$ in positive characteristic [HNR14]). Compact generation of $\mathrm{D}_{\mathrm{qc}}(X)$, however, is sufficient to prove that $\mathrm{D}(\mathrm{Q} \operatorname{Coh}(X)) \rightarrow \mathrm{D}_{\mathrm{qc}}(X)$ is an equivalence HNR14.

\section{QUASI-FINITE FLAT LOCALITY OF $\beta$-CRISPNESS AND APPLICATIONS}

We are now in a position to prove Theorems $\mathrm{A}, \mathrm{B}$, and $\mathrm{C}$ and address the applications mentioned in the Introduction.

Proof of Theorem $C$, Take $\mathcal{D}=\mathbf{R e p}^{\mathrm{fp}} / X$. By Examples 5.2, 5.6, 6.2 and 6.5, the $\mathcal{D}$-presheaf of triangulated categories $\mathrm{D}_{\mathrm{qc}}$ satisfies Conditions (1) $-(5)$ of Theorem 6.9. The result now follows from Lemma 4.10 and Theorem 6.9.

Proving Theorems $\mathrm{A}$ and $\mathrm{B}$ is now very simple.

Proof of Theorem A, By Ryd11, Thm. 7.1] there exists a locally quasi-finite flat morphism $p: X^{\prime} \rightarrow X$, where $X^{\prime}$ is a scheme. Since $X$ is quasi-compact, we may further assume that $X^{\prime}$ is an affine scheme, and consequently the morphism $p$ is also quasi-compact and separated. The result now follows by combining Example 8.5 with Theorem C.

Proof of Theorem $B$. Since $X$ is of s-global type, there exists an integer $N>0$, a quasi-affine $\mathbb{Q}$-scheme $V$ with an action of $\mathrm{GL}_{N}$, together with an étale, representable, separated and finitely presented morphism $p:\left[V / \mathrm{GL}_{N}\right] \rightarrow X$. Now the result follows from Theorem $\mathrm{C}$ and Example 8.6

We now recall some results of Sumihiro and Brion.

Proposition 9.1 (Sumihiro and Brion). Let $X$ be a variety over a field $k$. Let $G$ be an affine algebraic $k$-group scheme acting on $X$. Assume that either $X$ is

(1) geometrically normal, or

(2) quasi-projective and either

(a) geometrically seminormal, or

(b) $\operatorname{char} k=p>0$, or

(c) the action is linearizable, or

(d) $G^{0}$ is a torus.

Then there exists a finite field extension $k^{\prime} / k$, a quasi-projective variety $W^{\prime}$ over $k^{\prime}$ with a linear action of $G^{\prime}=\left(G \otimes_{k} k^{\prime}\right)_{\text {red }}^{0}$ and an étale $G^{\prime}$-equivariant morphism $f: W^{\prime} \rightarrow X_{k^{\prime}}$.

Proof. Choose a finite field extension $k^{\prime} / k$ such that $X^{\prime}=X \times_{k} k^{\prime}$ is normal (resp. seminormal, resp. $G^{\prime}$ is smooth, resp. $G^{\prime}$ is a split torus). By construction $G^{\prime}$ is then a smooth connected group scheme.

If $X^{\prime}$ is normal, then by Sumihiro's theorem, we can choose $f$ as a Zariski-open covering [Sum74, Lem. 8] (see Sum75, Thm. 3.8] when $k$ is not algebraically closed, or replace $k$ with a finite field extension). If $X^{\prime}$ is seminormal and quasi-projective, 
then an étale $f$ exists by Brion [Bri15, Thm. 4.7]. If char $k>0$, then an étale $f$ exists by [Bri15, §4.3]. If the action is already linearizable, then let $f$ be the identity. If $G^{\prime}$ is a split torus, then an étale $f$, with $W^{\prime}$ affine, exists by Bri15, Thm. 4.8].

In AHR15, Thm. 2.6] it is proved that if $G$ acts with linearly reductive stabilizers at closed points (e.g., if $G$ is linearly reductive), then the result of Proposition 9.1 holds for any algebraic space of finite type (not necessarily normal, quasi-projective or even separated). In particular, if $G^{0}$ is a torus, we may drop the requirement that $X$ is quasi-projective.

The Corollary of Theorem $B$ is a special case of the following.

Corollary 9.2. Let $(X, G, k)$, and $\left(W^{\prime}, G^{\prime}, k^{\prime}\right)$ be as in Proposition 9.1. Then

(1) $\left[W^{\prime} / G^{\prime}\right]$ has the resolution property;

(2) the map $\left[W^{\prime} / G^{\prime}\right] \rightarrow\left[X^{\prime} / G^{\prime}\right] \rightarrow[X / G]$ is quasi-finite and faithfully flat; and

(3) $[X / G]$ is of s-global type.

If in addition, char $k=0$ or $\left(G \otimes_{k} \bar{k}\right)_{\mathrm{red}}^{0}$ is a torus, then

(4) $\left[W^{\prime} / G^{\prime}\right]$ is concentrated and $\aleph_{0}$-crisp;

(5) $\mathrm{D}\left(\mathrm{Q} \operatorname{Coh}_{G}(X)\right)=\mathrm{D}_{\mathrm{qc}}([X / G])$ is compactly generated; and

(6) for every $G$-invariant open subset $U \subseteq X$, there exists a compact perfect $G$-equivariant complex with support exactly $X \backslash U$.

Proof. (1) is Example 7.5 (3), (2) is by construction, and (3) follows from (2) and Ryd15, Prop. 2.8(iii)]. Under the additional assumption on $k$ and $G$, we have that $B G^{\prime}$ is concentrated [HR15, Thm. B], hence so is $\left[W^{\prime} / G^{\prime}\right]$ and (4) follows from Proposition 8.4. It follows that $[X / G]$ is $\aleph_{0}$-crisp by Theorem C We always have that $\mathrm{QCoh}_{G}(X)=\mathrm{Q} \operatorname{Coh}([X / G])$ and we have that $\mathrm{D}(\mathrm{Q} \operatorname{Coh}([X / G]))=\mathrm{D}_{\mathrm{qc}}([X / G])$ since $\mathrm{D}_{\mathrm{qc}}([X / G])$ is compactly generated [HNR14].

Example 9.3 (Brauer groups). Let $X$ be a quasi-compact algebraic stack with quasi-finite and separated diagonal. Let $\alpha \in H^{2}\left(X, \mathbb{G}_{m}\right)$ be an element of the (bigger) cohomological Brauer group and let $X$ denote the $\mathbb{G}_{m}$-gerbe corresponding to $\alpha$. Since $X$ has quasi-finite diagonal, there exists a quasi-finite flat presentation $p: X^{\prime} \rightarrow X$ such that $X^{\prime}$ is affine and $p^{*} \alpha=0$. Indeed, recall that $X$ admits a quasi-finite flat presentation $q: U \rightarrow X$ by an affine scheme Ryd15, Thm. 7.1] and then we may trivialize $q^{*} \alpha$ by a further surjective étale morphism of schemes. In particular, $X \times_{X} X^{\prime}=X^{\prime} \times B \mathbb{G}_{m}$ has the resolution property and is cohomological affine. It follows that $\mathrm{D}_{\mathrm{qc}}\left(X \times_{X} X^{\prime}\right)$ and $\mathrm{D}_{\mathrm{qc}}(X)$ have countable sets of compact generators by Proposition 8.4 and Theorem $\mathrm{C}$.

Let $\mathcal{D}=\operatorname{Rep}^{\mathrm{fp}} / X$ and let $\mathcal{T}=\mathrm{D}\left(\mathrm{Q} \operatorname{Coh}^{\alpha}(-)\right)$ be the presheaf of derived categories of $\alpha$-twisted sheaves. Then the conditions of Theorem 6.9 are satisfied. Indeed, there is a canonical decomposition $\mathrm{D}_{\mathrm{qc}}\left(\mathcal{X} \times_{X} T\right)=\mathrm{D}\left(\mathrm{Q} \operatorname{Coh}\left(\mathcal{X} \times_{X} T\right)\right)=$ $\bigoplus_{m \in \mathbb{Z}} \mathrm{D}\left(\mathrm{Q}\right.$ Coh $\left.^{m \alpha}(T)\right)$ which is respected by pullback ${ }^{1}$. Since $\mathrm{D}_{\mathrm{qc}}\left(\mathcal{X}_{\times_{X}}-\right)$ satisfies conditions (1) - 5] of Theorem 6.9, so does $\mathcal{T}$.

Thus, $\mathrm{D}\left(\mathrm{Q} \operatorname{Coh}^{\alpha}\left(X^{\prime}\right)\right)=\mathrm{D}\left(\mathrm{Q} \operatorname{Coh}\left(X^{\prime}\right)\right)=\mathrm{D}_{\mathrm{qc}}\left(X^{\prime}\right)$ is compactly generated by 1 object with supports. It follows that $\mathrm{D}\left(\mathrm{Q} \operatorname{Coh}^{\alpha}(X)\right)$ is compactly generated by 1 object with supports (Theorem 6.9). The endomorphism algebra of this object, in a dg-enhancement of $\mathrm{D}\left(\mathrm{Q} \operatorname{Coh}^{\alpha}(X)\right)$, is a derived Azumaya algebra Toë12.

Alternatively, one could argue as follows. Take the degree 1 part of the compact generators of $\mathrm{D}_{\mathrm{qc}}(X)$. This gives a countable generating set $\left\{P_{i}\right\}$ of compact objects in $\mathrm{D}_{\mathrm{qc}}^{\alpha}(X)$. For sufficiently large $n>0$, the direct sum $P:=\bigoplus_{i=1}^{n} P_{i}$ gives

\footnotetext{
${ }^{1}$ Here we have tacitly used HNR14 to identify $\mathrm{D}_{\mathrm{qc}}\left(X_{\times_{X}} T\right)=\mathrm{D}\left(\mathrm{Q} \operatorname{Coh}\left(X_{\times_{X}} T\right)\right)$, which holds since $\mathrm{D}_{\mathrm{qc}}\left(X \times_{X} T\right)$ is compactly generated. In general, one could define $\mathrm{D}_{\mathrm{qc}}^{\alpha}(X)$ as the degree one part of $\mathrm{D}_{\mathrm{qc}}(X)$.
} 
a compact object that locally generates $\mathrm{D}_{\mathrm{qc}}^{\alpha}(X)$. Indeed, since $\mathrm{D}_{\mathrm{qc}}^{\alpha}\left(X^{\prime}\right)=\mathrm{D}_{\mathrm{qc}}\left(X^{\prime}\right)$ and $X^{\prime}$ is affine, it is sufficient that $\operatorname{supph}\left(p^{*} P\right)=\left|X^{\prime}\right|$ (Lemma 4.9). This compact local generator $P$ is enough to produce a derived Azumaya algebra Toë12, Prop. 4.6].

This latter argument also works for any quasi-compact and quasi-separated algebraic stack $X$ such that $\mathrm{D}_{\mathrm{qc}}(\mathcal{X})$ has a set of compact generators. In this case, take $p: X^{\prime} \rightarrow X$ as a smooth presentation by an affine scheme such that $p^{*} \alpha=0$. By [AHR15, Thm. 2.26], $\mathrm{D}_{\mathrm{qc}}(X)$ is compactly generated for any algebraic stack $X$ of finite type over a field, with affine diagonal, and linearly reductive stabilizers at closed points. We can thus conclude that for such $X$, every cohomological Brauer class comes from a derived Azumaya algebra.

Example 9.4 (Sheaves of linear categories on derived stacks). Let $\left(X, \mathcal{O}_{X}\right)$ be a derived (or spectral) Deligne-Mumford stack. The 0-truncation $\left(X, \pi_{0} \mathcal{O}_{X}\right)$ is an ordinary Deligne-Mumford stack with the same underlying topos $X$. In fact, even for a non-connective $\mathbb{E}_{\infty}$-algebra $A$, the category of étale $A$-algebras is equivalent to the category of étale $\pi_{0} A$-algebras [HA, Thm. 7.5.0.6].

Let $F \in \operatorname{QStk}(X)$ be a quasi-coherent stack on $X$ [DAGXI, §8], e.g., $F=$ QCoh $(X)$. For every object $U$ in the small étale topos of $X$, this gives an $\mathcal{O}_{X}(U)$ linear $\infty$-category $F(U)$. Let $h F$ be the presheaf of triangulated categories that assigns to each étale $U \rightarrow X$ the homotopy category of $F(U)$. Compact generation of $F(U)$ is a statement about its homotopy category [HA, Rem. 1.4.4.3]. Moreover, since the conditions (1)-(5) of Theorem 6.9 can all be verified étale-locally, it follows that Theorem 6.9 can be applied to $h F$ to deduce compact generation of $F(X)$ from local compact generation of $F$.

\section{Appendix A. Generators from above}

Let $w: W \rightarrow X$ be an étale, separated, finitely presented and representable morphism of algebraic stacks. Define

$$
\mathcal{P}^{\prime}(w)=\left\{\mathrm{R}\left(w_{\mathrm{qc}}\right)_{*} \mathrm{R}\left(u_{\mathrm{qc}}\right)_{*} P:(u: U \rightarrow W) \in \mathbf{R e p}^{\text {fp,ét,sep }} / W \text { and } P \in \mathrm{D}_{\mathrm{qc}}(U)^{c}\right\}
$$

and let $\mathcal{P}(w) \subseteq \mathrm{D}_{\mathrm{qc}}(X)$ be the smallest thick subcategory of $\mathrm{D}_{\mathrm{qc}}(X)$ containing $\mathcal{P}^{\prime}(w)$. The following Lemma - requested by Neeman - is a natural generalization to algebraic stacks of a result that has found applications to Grothendieck duality for schemes Nee14, Lem. 3.1].

Lemma A.1. Let $w: W \rightarrow X$ be an étale, separated, finitely presented, representable and surjective morphism of algebraic stacks. If $X$ is quasi-compact and quasi-separated and $\mathrm{D}_{\mathrm{qc}}(X)$ is compactly generated, then

$$
\mathrm{D}_{\mathrm{qc}}(X)^{c} \subseteq \mathcal{P}(w)
$$

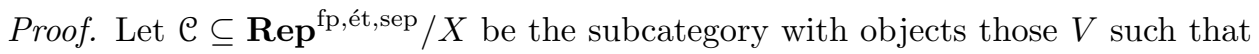
$\mathrm{D}_{\mathrm{qc}}(V)^{c} \subseteq \mathcal{P}\left(W \times_{X} V \rightarrow V\right)$. Note that if $W \times_{X} V \rightarrow V$ admits a section, then it is clear that $V \in \mathcal{C}$. In particular, it follows immediately that $W \in \mathcal{C}$. Now we will prove that $X \in \mathcal{C}$ using Ryd11, Thm. 6.1]. To do this, we need to verify the following three conditions for a morphism $v: V^{\prime} \rightarrow V$ in $\mathbf{R e p}^{\text {fp,ét,sep }} / X$.

(D1) If $V \in \mathcal{C}$, then $V^{\prime} \in \mathcal{C}$;

(D2) if $V^{\prime} \in \mathcal{C}$ and $v$ is finite and surjective, then $V \in \mathcal{C}$; and

(D3) if $v$ is an étale neighborhood of $V \backslash U$, where $j: U \rightarrow V$ is an open immersion in $\boldsymbol{R e p}^{\text {fp,ét,sep }} / X$, and $U$ and $V^{\prime}$ belong to $\mathcal{C}$, then $V \in \mathcal{C}$.

For (D1): Lemma 8.2 (and Theorem 3.12 imply that $\mathrm{D}_{\mathrm{qc}}\left(V^{\prime}\right)^{c}$ is the smallest thick subcategory of $\mathrm{D}_{\mathrm{qc}}\left(V^{\prime}\right)$ containing $\mathrm{L} v_{\mathrm{qc}}^{*} Q$, where $Q \in \mathrm{D}_{\mathrm{qc}}(V)^{c}$. A simple 
argument involving flat base change (Theorem 2.6 [4) and the preservation of compact objects under concentrated morphisms (Example 3.9 now shows that $V^{\prime} \in \mathcal{C}$. For (D2): Proposition 6.6 (and Theorem 3.12) imply that $\mathrm{D}_{\mathrm{qc}}(V)^{c}$ is the smallest thick subcategory of $\mathrm{D}_{\mathrm{qc}}(V)$ containing the collection of complexes $\mathrm{R}\left(v_{\mathrm{qc}}\right)_{*} Q$, where $Q \in \mathrm{D}_{\mathrm{qc}}\left(V^{\prime}\right)^{c}$. The property now follows from the trivial observation that

$$
\left\{\mathrm{R}\left(v_{\mathrm{qc}}\right)_{*} M: M \in \mathcal{P}\left(W \times_{X} V^{\prime} \rightarrow V^{\prime}\right)\right\} \subseteq \mathcal{P}\left(W \times_{X} V \rightarrow V\right) .
$$

For (D3): we note that the Mayer-Vietoris triangle of Lemma 5.9 11 implies that if $P \in \mathrm{D}_{\mathrm{qc}}(V)^{c}$, then there is a distinguished triangle:

$$
P \rightarrow \mathrm{R}\left(j_{\mathrm{qc}}\right)_{*} \mathrm{~L} j_{\mathrm{qc}}^{*} P \oplus \mathrm{R}\left(f_{\mathrm{qc}}\right)_{*} \mathrm{~L} f_{\mathrm{qc}}^{*} P \rightarrow \mathrm{R}\left(f_{\mathrm{qc}}\right)_{*} \mathrm{~L} f_{\mathrm{qc}}^{*} \mathrm{R}\left(j_{\mathrm{qc}}\right)_{*} \mathrm{~L} j_{\mathrm{qc}}^{*} P \rightarrow P[1] .
$$

Since $\mathrm{L} f_{\mathrm{qc}}^{*} \mathrm{R}\left(j_{\mathrm{qc}}\right)_{*} \simeq \mathrm{R}\left(j_{\mathrm{qc}}^{\prime}\right)_{*} \mathrm{~L} f_{\mathrm{qc}}^{\prime *}$, where $j^{\prime}: U^{\prime}=U \times_{V} V^{\prime} \rightarrow V^{\prime}$ and $f^{\prime}: U^{\prime} \rightarrow U$ are the projections, it follows immediately that $\mathrm{D}_{\mathrm{qc}}(V)^{c}$ is contained in the smallest thick subcategory of $\mathrm{D}_{\mathrm{qc}}(V)$ containing the objects:

- $\left\{\mathrm{R}\left(j_{\mathrm{qc}}\right)_{*} M: M \in \mathcal{P}\left(W \times_{X} U \rightarrow U\right)\right\}$,

- $\left\{\mathrm{R}\left(f_{\mathrm{qc}}\right)_{*} M: M \in \mathcal{P}\left(W \times_{X} V^{\prime} \rightarrow V^{\prime}\right)\right\}$, and

- $\left\{\mathrm{R}\left(\left(f \circ j^{\prime}\right)_{\mathrm{qc}}\right)_{*} M: M \in \mathcal{P}\left(W \times_{X} U^{\prime} \rightarrow U^{\prime}\right)\right\}$.

But all the objects above are contained in $\mathcal{P}\left(W \times_{X} V \rightarrow V\right)$ and the claim follows. Since $W \in \mathcal{C}$, we may now conclude that $X \in \mathcal{C}$.

\section{REFERENCES}

[AG14] B. Antieau and D. Gepner, Brauer groups and étale cohomology in derived algebraic geometry, Geom. Topol. 18 (2014), no. 2, 1149-1244.

[AHR15] J. Alper, J. Hall, and D. Rydh, A Luna étale slice theorem for algebraic stacks, April 2015, arXiv:1504.06467.

[AJPV17] L. Alonso Tarrío, A. Jeremías López, M. Pérez Rodríguez, and M. J. Vale Gonsalves, On the derived category of quasi-coherent sheaves on an Adams geometric stack, J. Pure Appl. Algebra (2017), arXiv:1604.06018 To appear, available online.

[Alp13] J. Alper, Good moduli spaces for Artin stacks, Ann. Inst. Fourier (Grenoble) 63 (2013), no. $6,2349-2402$.

[Ant14] B. Antieau, A local-global principle for the telescope conjecture, Adv. Math. 254 (2014), 280-299.

[Aus66] M. Auslander, Coherent functors, Proc. Conf. Categorical Algebra (La Jolla, Calif., 1965), Springer, New York, 1966, pp. 189-231.

[BB03] A. Bondal and M. Van den Bergh, Generators and representability of functors in commutative and noncommutative geometry, Mosc. Math. J. 3 (2003), no. 1, 1-36, 258.

[Beh03] K. Behrend, Derived l-adic categories for algebraic stacks, Mem. Amer. Math. Soc. 163 (2003), no. 774 , viii +93.

[BLS16] D. Bergh, V. A. Lunts, and O. M. Schnürer, Geometricity for derived categories of algebraic stacks, Selecta Math. (N.S.) 22 (2016), 2535-2568.

[BN93] M. Bökstedt and A. Neeman, Homotopy limits in triangulated categories, Compositio Math. 86 (1993), no. 2, 209-234.

[Bra14] M. Brandenburg, Tensor categorical foundations of algebraic geometry, Ph.D. thesis, Wilhelms-Universität Münster, May 2014, p. 243.

[Bri15] M. Brion, On linearization of line bundles, J. Math. Sci. Univ. Tokyo 22 (2015), no. 1, 113-147.

[BZFN10] D. Ben-Zvi, J. Francis, and D. Nadler, Integral transforms and Drinfeld centers in derived algebraic geometry, J. Amer. Math. Soc. 23 (2010), no. 4, 909-966.

[CS16] A. Canonaco and P. Stellari, Uniqueness of dg enhancements for the derived category of a Grothendieck category, J. Eur. Math. Soc. (2016), to appear.

[DAGXI] J. Lurie, Derived Algebraic Geometry XI: Descent Theorems, available on homepage, Sep 2011.

[DAGXII] J. Lurie, Derived Algebraic Geometry XII: Proper Morphisms, Completions, and the Grothendieck Existence Theorem, available on homepage, Nov 2011.

[DG13] V. Drinfeld and D. Gaitsgory, On some finiteness questions for algebraic stacks, Geom. Funct. Anal. 23 (2013), no. 1, 149-294. 
[DM12] U. V. Dubey and V. M. Mallick, Spectrum of some triangulated categories, J. Algebra 364 (2012), 90-118.

[EGA] A. Grothendieck, Éléments de géométrie algébrique, I.H.E.S. Publ. Math. 4, 8, 11, 17, 20, 24, 28, 32 (1960, 1961, 1961, 1963, 1964, 1965, 1966, 1967).

[FL85] W. Fulton and S. Lang, Riemann-Roch algebra, Grundlehren der Mathematischen Wissenschaften [Fundamental Principles of Mathematical Sciences], vol. 277, SpringerVerlag, New York, 1985.

[Gab81] Ofer Gabber, Some theorems on Azumaya algebras, The Brauer group (Sem., Les Plans-sur-Bex, 1980), Lecture Notes in Math., vol. 844, Springer, Berlin-New York, 1981, pp. 129-209.

[GR17] D. Gaitsgory and N. Rozenblyum, A Study in Derived Algebraic Geometry: Volume I: Correspondences and Duality, Mathematical Surveys and Monographs, vol. 221, American Mathematical Society, Providence, RI, 2017.

[Gro10] P. Gross, Vector bundles as generators on schemes and stacks, Ph.D. thesis, HeinrichHeine-Universität Düsseldorf, May 2010.

[Gro17] P. Gross, Tensor generators on schemes and stacks, Algebr. Geom. 4 (2017), arXiv:1306.5418 to appear.

[HA] J. Lurie, Higher Algebra, available on homepage, May 2016.

[HAGII] B. Toën and G. Vezzosi, Homotopical algebraic geometry. II. Geometric stacks and applications, Mem. Amer. Math. Soc. 193 (2008), no. 902, x+224.

[Hal14] J. Hall, Cohomology and base change for algebraic stacks, Math. Z. 278 (2014), no. 1-2, 401-429.

[Hal16] J. Hall, The Balmer spectrum of a tame stack, Ann. K-Theory 1 (2016), no. 3, 259-274.

[Hal17] J. Hall, Openness of versality via coherent functors, J. Reine Angew. Math. 722 (2017), $137-182$.

[Har98] R. Hartshorne, Coherent functors, Adv. Math. 140 (1998), no. 1, 44-94.

[HNR14] J. Hall, A. Neeman, and D. Rydh, One positive and two negative results for derived categories of algebraic stacks, preprint, May 2014, arXiv:1405.1888v2

[HPS97] M. Hovey, J. H. Palmieri, and N. P. Strickland, Axiomatic stable homotopy theory, Mem. Amer. Math. Soc. 128 (1997), no. 610, x+114.

[HR15] J. Hall and D. Rydh, Algebraic groups and compact generation of their derived categories of representations, Indiana Univ. Math. J. 64 (2015), no. 6, 1903-1923.

[HR16] J. Hall and D. Rydh, Mayer-Vietoris squares in algebraic geometry, June 2016, arXiv:1606.08517.

[HR17] J. Hall and D. Rydh, The telescope conjecture for algebraic stacks, J. Topol. 10 (2017), no. $3,776-794$.

[HTT] J. Lurie, Higher topos theory, Annals of Mathematics Studies, vol. 170, Princeton University Press, Princeton, NJ, 2009.

[Jon03] A. J. de Jong, A result of Gabber, preprint available at http://www.math.columbia. edu/ dejong/ 2003, p. 9.

[Kri09] A. Krishna, Perfect complexes on Deligne-Mumford stacks and applications, J. KTheory 4 (2009), no. 3, 559-603.

[KS06] M. Kashiwara and P. Schapira, Categories and sheaves, Grundlehren der Mathematischen Wissenschaften [Fundamental Principles of Mathematical Sciences], vol. 332, Springer-Verlag, Berlin, 2006.

[Lie04] M. Lieblich, Moduli of twisted sheaves and generalized Azumaya algebras, ProQuest LLC, Ann Arbor, MI, 2004, Thesis (Ph.D.)-Massachusetts Institute of Technology.

[Lip09] J. Lipman, Notes on derived functors and Grothendieck duality, Foundations of Grothendieck duality for diagrams of schemes, Lecture Notes in Math., vol. 1960, Springer, Berlin, 2009, pp. 1-259.

[LMB] G. Laumon and L. Moret-Bailly, Champs algébriques, Ergebnisse der Mathematik und ihrer Grenzgebiete. 3. Folge., vol. 39, Springer-Verlag, Berlin, 2000.

[LN07] J. Lipman and A. Neeman, Quasi-perfect scheme-maps and boundedness of the twisted inverse image functor, Illinois J. Math. 51 (2007), no. 1, 209-236.

[LO08] Y. Laszlo and M. Olsson, The six operations for sheaves on Artin stacks. I. Finite coefficients, Publ. Math. Inst. Hautes Études Sci. 107 (2008), 109-168.

[Lur04] J. Lurie, Tannaka duality for geometric stacks, preprint, December 2004, arXiv:math/0412266 p. 14.

[LZ12] Y. Liu and W. Zheng, Enhanced six operations and base change theorem for Artin stacks, November 2012, arXiv:1211.5948

[MB96] L. Moret-Bailly, Un problème de descente, Bull. Soc. Math. France 124 (1996), no. 4, 559-585. 
[Nee92a] A. Neeman, The chromatic tower for $D(R)$, Topology 31 (1992), no. 3, 519-532, With an appendix by Marcel Bökstedt.

[Nee92b] A. Neeman, The connection between the K-theory localization theorem of Thomason, Trobaugh and Yao and the smashing subcategories of Bousfield and Ravenel, Ann. Sci. École Norm. Sup. (4) 25 (1992), no. 5, 547-566.

[Nee96] A. Neeman, The Grothendieck duality theorem via Bousfield's techniques and Brown representability, J. Amer. Math. Soc. 9 (1996), no. 1, 205-236.

[Nee01a] A. Neeman, On the derived category of sheaves on a manifold, Doc. Math. 6 (2001), 483-488 (electronic).

[Nee01b] A. Neeman, Triangulated categories, Annals of Mathematics Studies, vol. 148, Princeton University Press, Princeton, NJ, 2001

[Nee11] A. Neeman, Non-left-complete derived categories, Math. Res. Lett. 18 (2011), no. 5, $827-832$.

[Nee14] A. Neeman, An improvement on the base-change theorem and the functor $f^{!}$, June 2014, arXiv:1406.7599

[Ols07] M. Olsson, Sheaves on Artin stacks, J. Reine Angew. Math. 603 (2007), 55-112.

[Ryd11] D. Rydh, Étale dévissage, descent and pushouts of stacks, J. Algebra 331 (2011), $194-223$.

[Ryd15] D. Rydh, Noetherian approximation of algebraic spaces and stacks, J. Algebra 422 (2015), 105-147.

[SAG] J. Lurie, Spectral Algebraic Geometry, available on homepage, Oct 2016

[SGA6] Théorie des intersections et théorème de Riemann-Roch, Lecture Notes in Mathematics, Vol. 225, Springer-Verlag, Berlin, 1971, Séminaire de Géométrie Algébrique du Bois-Marie 1966-1967 (SGA 6), Dirigé par P. Berthelot, A. Grothendieck et L. Illusie. Avec la collaboration de D. Ferrand, J. P. Jouanolou, O. Jussila, S. Kleiman, M. Raynaud et J. P. Serre.

[Stacks] The Stacks Project Authors, Stacks Project, http://stacks.math.columbia.edu

[Sum74] H. Sumihiro, Equivariant completion, J. Math. Kyoto Univ. 14 (1974), 1-28.

[Sum75] H. Sumihiro, Equivariant completion. II, J. Math. Kyoto Univ. 15 (1975), no. 3, 573605 .

[Tho87] R. W. Thomason, Equivariant resolution, linearization, and Hilbert's fourteenth problem over arbitrary base schemes, Adv. in Math. 65 (1987), no. 1, 16-34.

[Tho97] R. W. Thomason, The classification of triangulated subcategories, Compositio Math. 105 (1997), no. 1, 1-27.

[Toë12] B. Toën, Derived Azumaya algebras and generators for twisted derived categories, Invent. Math. 189 (2012), no. 3, 581-652.

[Tot04] B. Totaro, The resolution property for schemes and stacks, J. Reine Angew. Math. $\mathbf{5 7 7}$ (2004), 1-22.

[TT90] R. W. Thomason and T. Trobaugh, Higher algebraic K-theory of schemes and of derived categories, The Grothendieck Festschrift, Vol. III, Progr. Math., vol. 88, Birkhäuser Boston, Boston, MA, 1990, pp. 247-435.

Department of Mathematics, University of Arizona, Tucson, AZ 85721-0089, USA

E-mail address: jackhall@math.arizona.edu

KTH Royal Institute of Technology, Department of Mathematics, SE-100 44 StockHOLM, SWEDEN

E-mail address: dary@math.kth.se 